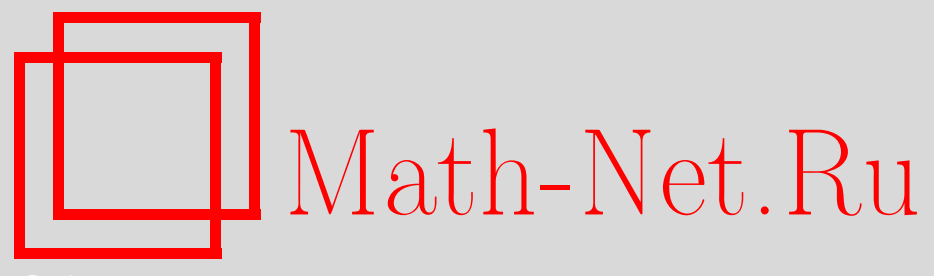

А. Г. Баскаков, Исследование линейных дифференциальных уравнений методами спектральной теории разностных операторов и линейных отношений, УМН, 2013, том 68 , выпуск 1, 77-128

DOI: https://doi.org/10.4213/rm9505

Использование Общероссийского математического портала Math-Net.Ru подразумевает, что вы прочитали и согласны с пользовательским соглашением http://www.mathnet.ru/rus/agreement

Параметры загрузки:

IP : 44.207 .124 .84

26 апреля 2023 г., $15: 27: 45$

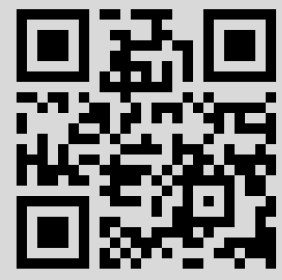




\title{
Исследование линейных дифференциальных уравнений методами спектральной теории разностных операторов и линейных отношений
}

\begin{abstract}
А. Г. Баскаков
Многие свойства решений (ограниченность, почти периодичность, устойчивость) линейных дифференциальных уравнений с неограниченными операторными коэффициентами тесно связаны с соответствующими свойствами дифференциального оператора, определяющего рассматриваемое уравнение и действующего в подходящем функциональном пространстве. Его свойства обратимости, корректности, фредгольмовости, а также структура спектра зависят от размерности ядра, коразмерности образа, их дополняемости. Вводится понятие состояния линейного отношения (многозначного линейного оператора), с которым ассоциируется совокупность свойств его ядра и образа. Изучаемому дифференциальному оператору (соответствующему уравнению) ставится в соответствие линейный разностный оператор (разностное отношение), и доказываются утверждения о совпадении множества их состояний, а также необходимые и достаточные условия их фредгольмовости. Получены критерии почти периодичности на бесконечности решений дифференциальных уравнений. При доказательстве основных результатов существенно используется свойство экспоненциальной дихотомии семейства эволюционных операторов и спектральная теория линейных отношений.

Библиография: 97 названий.
\end{abstract}

Ключевые слова: линейные дифференциальные операторы, множество состояний оператора, фредгольмов оператор, разностные операторы и отношения, спектр оператора и линейного отношения, почти периодические на бесконечности функции.

DOI: $10.4213 /$ umn9505

\section{СОДЕРЖАНИЕ}

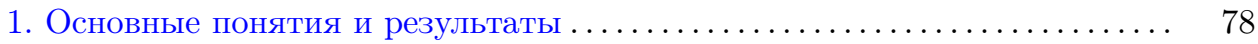

2. Однородные пространства функций ...................... 93

2.1. Основные функциональные пространства ................. 93

2.2. Однородные пространства функций и последовательностей .... . 94

Работа выполнена при поддержке РФФИ (гранты № 10-01-00276, 13-01-00378).

(C) А. Г. БАСКАКов, 
3. О состояниях оператора $\mathscr{N}_{a}^{+}$и отношения $\mathscr{D}_{E}^{+}$; доказательство тео-

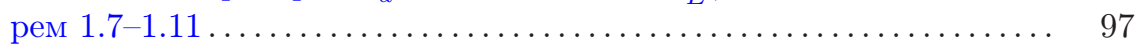

3.1. О ядрах и образах оператора $\mathscr{N}_{a}^{+}$и отношения $\mathscr{D}_{E}^{+} \ldots \ldots \ldots \ldots .97$

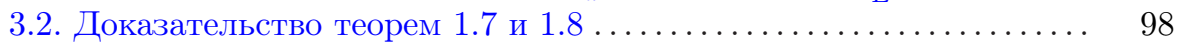

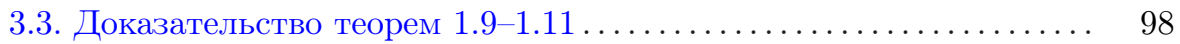

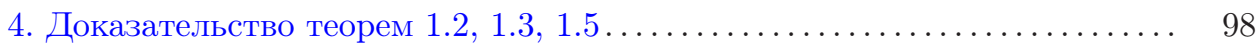

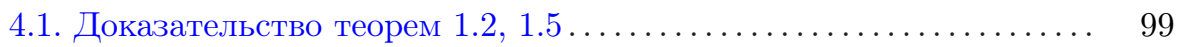

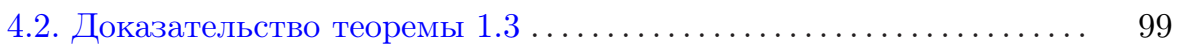

5. О состояниях оператора $\mathscr{D}$ и узлового оператора $\mathscr{N}_{b, a} ;$ доказательство

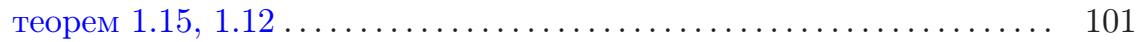

5.1. Доказательство теоремы $1.15 \ldots \ldots \ldots \ldots \ldots \ldots \ldots \ldots \ldots \ldots \ldots \ldots . \ldots \ldots$

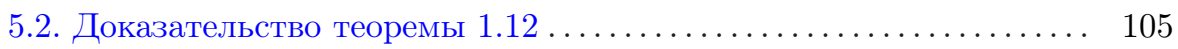

6. Критерии почти периодичности решений дифференциальных уравнений 110

7. Комментарии к основным понятиям и некоторым результатам из раз-

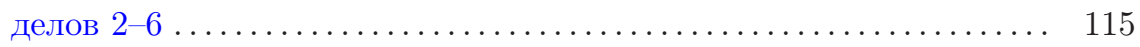

7.1. О выборе пространств и терминологии .................. 115

7.2. Комментарии к основным результатам ............... 117

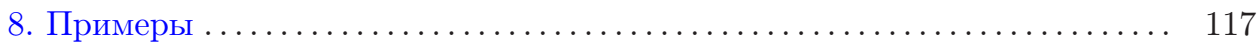

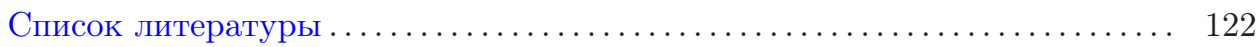

\section{1. Основные понятия и результаты}

Рассматривается линейное дифференциальное уравнение

$$
-\frac{d x}{d t}+A(t) x=f(t), \quad t \in \mathbb{J},
$$

где $\mathbb{J}$ - бесконечный промежуток вещественной оси $\mathbb{R}$ и $A(t): D(A(t)) \subset X \rightarrow X$, $t \in \mathbb{J},-$ семейство линейных замкнутых операторов, действующих в комплексном банаховом пространстве $X$. Пространство $X$ иногда будет называться $\oint a-$ зовым.

Предполагается корректная разрешимость задачи Коши $x(s)=x_{0} \in D(A(s))$, $t \geqslant s, t, s \in \mathbb{J}$, для однородного дифференциального уравнения

$$
\frac{d x}{d t}=A(t) x, \quad t \in \mathbb{J} .
$$

Она ведет к существованию семейства эволюционных операторов $\mathscr{U}: \Delta_{\mathbb{J}} \rightarrow$ End $X$, где $\Delta_{\mathbb{J}}=\{(t, s) \in \mathbb{J} \times \mathbb{J} \mid s \leqslant t\}$ и End $X$ - банахова алгебра ограниченных линейных операторов, действующих в $X$. По семейству $\mathscr{U}$ строится дифференциальный оператор

$$
L=L_{\mathscr{U}}=-\frac{d}{d t}+A(t)
$$

который действует в однородном пространстве $\mathscr{F}(\mathbb{J}, X)$ функций (см. раздел 2), определенных на $\mathbb{J}$ и принимающих значения в $X$. Одним из таких пространств является банахово пространство $C_{b}(\mathbb{J}, X)$ непрерывных и ограниченных на $\mathbb{J}$ функций. 
Данная статья посвящена вопросам существования и единственности ограниченных решений для дифференциального уравнения (1.1), где функция $f$ принадлежит одному из однородных пространств функций $\mathscr{F}(\mathbb{J}, X)$ (в частности, для ограниченной функции $f$ ). Такие вопросы относят к геометрической, или качественной, теории дифференциальных уравнений, истоки которой восходят к Пуанкаре и Ляпунову.

С вопросами существования и единственности ограниченных решений тесно связаны вопросы устойчивости решений дифференциальных уравнений. Отправляясь от идей и результатов А. М. Ляпунова, М. Г. Крейн в статье [1], а затем в ротапринтном издании [2] заметил, что многие факты теории устойчивости решений можно получить, используя теорию операторов, действующих в банаховых пространствах. Отказ от специфической теории операторов (матричного анализа) в конечномерном пространстве сделал более прозрачными и простыми многие доказательства и конструкции. Таким образом, общий подход оказал положительное влияние и на классическую теорию (см. монографии Б. П. Демидовича [3], У. Коппеля [4], Ф. Хартмана [5]). Отметим, что исследование линейных дифференциальных уравнений имеет не только самостоятельное значение, но и служит базой для изучения нелинейных уравнений по линейному приближению.

Результаты, представленные в монографиях Х. Л. Массера, Х. Х. Шеффеpa [6], Ю.Л. Далецкого, М. Г. Крейна [7], относились к случаю, когда операторы $A(t), t \in \mathbb{J}$, являются ограниченными операторами, действующими в банаховом пространстве. В связи с приложениями к параболическим дифференциальным уравнениям в частных производных остро стоял вопрос (он ставился в обеих монографиях) об исследовании качественных свойств решений с неограниченными операторными коэффициентами: "Авторы отчетливо сознают, что арена бесконечномерных пространств требует присутствия неограниченных операторов, без которых невозможна настоящая теория устойчивости систем с бесконечным числом степеней свободы” (см. [7]), “. . мы совершенно игнорируем возможность распространения теории на случай, когда значения $A$ суть неограниченные операторы в $X$. Такое обобщение теории представило бы, конечно, огромный интерес, особенно ввиду возможных приложений к уравнениям в частных производных" (см. [6]).

Существенный вклад в теорию рассматриваемых здесь уравнений был сделан В. В. Жиковым в статье [8] (см. также [9]), где была доказана эквивалентность условия обратимости оператора $L=-d / d t+A(t)$ в пространстве $C_{b}(\mathbb{R}, X)$ условию экспоненциальной дихотомии решений уравнения (1.2). Отметим также монографию Д. Хенри [10], где строилась геометрическая теория полулинейных параболических уравнений, которая существенно использует свойство обратимости оператора $L=-d / d t+A(t)$ в банаховом пространстве $C_{b}(\mathbb{R}, X)$ в предположении, что $A(t), t \in \mathbb{R},-$ секториальные операторы.

Следует отметить, что исследования по корректной разрешимости задачи Коши для уравнения вида (1.2) существенно опережали соответствующие исследования по качественной теории уравнений $(1.1),(1.2)$. Случаю, когда $A(t) \equiv A, t \in \mathbb{R}_{+}=[0, \infty),-$ постоянная операторная функция, отвечает теория полугрупп операторов (см. [11], [12]). В знаменитой теореме Хилле-Филлип- 
са-Иосида-Феллера-Миядеры (см. [11; теорема 12.3.1], [12; теорема 3.3.8]) получено необходимое и достаточное условие корректной разрешимости задачи Коши для дифференциального уравнения (1.2), используя резольвенту оператора $A: D(A) \subset X \rightarrow X$. В монографии [12] приведено большое число примеров уравнений в частных производных параболического типа, для которого соответствующий оператор является генератором сильно непрерывной полугруппы операторов (порождает корректную задачу Коши).

Современное состояние рассматриваемых в данной статье проблем тесно связано с использованием методов спектральной теории линейных замкнутых операторов, линейных отношений (многозначных линейных операторов), разностных операторов и отношений, полугрупп операторов.

Использование теории полугрупп операторов в геометрической теории дифференциальных уравнений вида (1.1), (1.2) осуществлялось в статьях [13]-[17]. Изучаемый (порождаемый уравнением (1.1)) дифференциальный оператор является генератором сильно непрерывной полугруппы разностных операторов. Таким образом, появилась возможность использования теории полугрупп операторов в качественной теории дифференциальных уравнений. Состояние соответствующей теории до 1999 г. подробно освещено в монографии К. Чиконе и Ю. Латушкина [18]. Для исследования дифференциальных операторов (уравнений), рассматриваемых в пространствах функций на полуоси, в статье [19] стали использоваться полугруппа разностных линейных отношений и линейные разностные отношения.

Исследование оператора $L$ (дифференциального уравнения (1.1)) и более общих операторов, порожденных семейством эволюционных операторов, проводится с существенным использованием разностных операторов и разностных линейных отношений, определенных на соответствующих однородных пространствах векторных последовательностей. Вводится понятие множества состояний линейных отношений (операторов) для описания определенных свойств ядра и образа линейного отношения (оператора) и тем самым проявляется степень его непрерывной обратимости. В частности, получены условия фредгольмовости разностных операторов, отношений и дифференциальных операторов. Полученные результаты тесно связаны с вопросами асимптотического поведения решений, устойчивости решений, проблемой существования ограниченных решений дифференциальных уравнений (1.1) и (1.2).

Сформулируем наиболее используемые понятия и определения (их достаточно для понимания статьи) и приведем некоторые из основных результатов.

В статье существенно используется понятие линейного отношения. Поэтому в разделе 3 приводятся некоторые понятия результатов из теории линейных отношений. Необходимость в таких понятиях возникает и при формулировании многих результатов статьи.

Пусть $\mathscr{X}, \mathscr{Y}$ - банаховы пространства, $\operatorname{Hom}(\mathscr{X}, \mathscr{Y})$ - банахово пространство линейных ограниченных операторов, действующих из $\mathscr{X}$ в $\mathscr{Y}$, End $\mathscr{X}=$ $\operatorname{Hom}(\mathscr{X}, \mathscr{X})$.

Любое линейное подпространство $\mathscr{A}$ из декартова произведения $\mathscr{X} \times \mathscr{Y}$ пространств $\mathscr{X}$ и $\mathscr{Y}$ называется линейным отношением между $\mathscr{X}$ и $\mathscr{Y ~ ( л и н е и ̆ н ы м ~}$ 
отношением на $\mathscr{X}$, если $\mathscr{X}=\mathscr{Y})$. Множество всех линейных отношений между $\mathscr{X}$ и $\mathscr{Y}$ обозначим символом $L R(\mathscr{X}, \mathscr{Y})$ (через $L R(\mathscr{X})$, если $\mathscr{Y}=\mathscr{X}$ ). Линейный оператор $A: D(A) \subset \mathscr{X} \rightarrow \mathscr{Y}$ часто отождествляется с (линейным) отношением $\mathscr{A}$ из $L R(\mathscr{X}, \mathscr{Y})$, совпадающим с графиком оператора $A$. Множество замкнутых линейных отношений из $L R(\mathscr{X}, \mathscr{Y})$ обозначается через $\operatorname{LRC}(\mathscr{X}, \mathscr{Y})$.

Для любого отношения $\mathscr{A} \in L R(\mathscr{X}, \mathscr{Y})$ определено обратное отношение $\mathscr{A}^{-1}=\{(y, x) \in \mathscr{Y} \times \mathscr{X} \mid(x, y) \in \mathscr{A}\} \in L R(\mathscr{Y}, \mathscr{X})$. Отношение $\operatorname{LRC}(\mathscr{X}, \mathscr{Y})$ называется непреръвно обратимъм, если $\mathscr{A}^{-1} \in \operatorname{Hom}(\mathscr{Y}, \mathscr{X})$, что эквивалентно одновременному выполнению условий: ядро $\operatorname{Ker} \mathscr{A}=\{x \in \mathscr{X} \mid(x, 0) \in \mathscr{A}\}$ отношения $\mathscr{A}$ нулевое и образ $\operatorname{Im} \mathscr{A}=\{y \in \mathscr{Y} \mid$ существует элемент $x \in$ $\mathscr{X}$ такой, что $(x, y) \in \mathscr{A}\}$ совпадает с $\mathscr{Y}$.

Через $D(\mathscr{A})$ обозначим область определения отношения $\mathscr{A} \in L R(\mathscr{X}, \mathscr{Y})$, т. е. $D(\mathscr{A})=\{x \in \mathscr{X} \mid(x, y) \in \mathscr{A}$ для некоторого $y \in \mathscr{Y}\}$. Через $\mathscr{A} x$, где $x \in D(\mathscr{A})$, обозначим множество $\{y \in \mathscr{Y} \mid(x, y) \in \mathscr{A}\}$ и положим $\|\mathscr{A} x\|=\inf _{y \in \mathscr{A} x}\|y\|$. Если $\mathscr{A} \in L R C(\mathscr{X}, \mathscr{Y})$, то $D(\mathscr{A})$ - банахово пространство с нормой графика $\|x\|_{\mathscr{A}}=\|x\|+\|\mathscr{A} x\|$.

В формулировке основных результатов статьи существенно используются приводимые далее определения.

ОПРЕДЕЛЕНИЕ 1.1. Пусть $\mathscr{A} \in \operatorname{LRC}(\mathscr{X}, \mathscr{Y})$. Рассмотрим следующие условия:

1) $\operatorname{Ker} \mathscr{A}=\{0\}$ (т. е. отношение $\mathscr{A}$ инъективно);

2) $1 \leqslant n=\operatorname{dim} \operatorname{Ker} \mathscr{A} \leqslant \infty$;

3) $\operatorname{Ker} \mathscr{A}$ - дополняемое подпространство либо в $D(\mathscr{A})$ (с нормой графика), либо в $\mathscr{X}$;

4) $\overline{\operatorname{Im} \mathscr{A}}=\operatorname{Im} \mathscr{A}$, что эквивалентно положительности величины (минимального модуля отношения $\mathscr{A}$ )

$$
\gamma(\mathscr{A})=\inf _{x \in D(\mathscr{A}) \backslash \operatorname{Ker} \mathscr{A}} \frac{\|\mathscr{A} x\|}{\operatorname{dist}(x, \operatorname{Ker} \mathscr{A})}=\inf _{\substack{(x, y) \in \mathscr{A} \\ x \in \operatorname{Ker} \mathscr{A}}} \frac{\|y\|}{\operatorname{dist}(x, \operatorname{Ker} \mathscr{A})},
$$

где $\operatorname{dist}(x, \operatorname{Ker} \mathscr{A})=\inf _{x_{0} \in \operatorname{Ker} \mathscr{A}}\left\|x-x_{0}\right\|$;

$5)$ отношение $\mathscr{A}$ корректно (равномерно инъективно), т. е. $\operatorname{Ker} \mathscr{A}=\{0\}$ и $\gamma(\mathscr{A})>0$

6) $\operatorname{Im} \mathscr{A}$ - замкнутое дополняемое в $\mathscr{Y}$ подпространство (в частности, величина (1.3) положительна);

7) $\operatorname{Im} \mathscr{A}$ - замкнутое подпространство из пространства $\mathscr{Y}$ коразмерности $1 \leqslant m=\operatorname{codim} \operatorname{Im} \mathscr{A} \leqslant \infty$;

8) $\operatorname{Im} \mathscr{A}=\mathscr{Y}$, т. е. $\mathscr{A}$ - сюръективное отношение;

9) отношение $\mathscr{A}$ непрерывно обратимо.

Если для отношения $\mathscr{A}$ выполнены все условия из совокупности условий $S_{0}=\left\{i_{1}, \ldots, i_{k}\right\}$, где $1 \leqslant i_{1}<\cdots<i_{k} \leqslant 9$, то будем говорить, что $\mathscr{A}$ находится в состоянии $S_{0}$. Множество состояний отношения $\mathscr{A}$ обозначим символом $\operatorname{St}_{\text {inv }}(\mathscr{A})$. 
ОПРЕДЕЛЕНИЕ 1.2. Если отношение $\mathscr{A} \in L R C(\mathscr{X}, \mathscr{Y})$ находится в одном из состояний $\{1,7\},\{2,7\},\{2,8\}$, где $n, m<\infty$, то оно называется фредгольмовым или $\Phi$-отношением. Если отношение $\mathscr{A}$ находится в одном из состояний $\{1,7\},\{2,7\},\{2,8\}$, где только одно из чисел $m, n$ конечно, то оно называется полуфредгольмовым ( $\Phi_{+}$-отношением, если $n<\infty$, и $\Phi_{-}$-отношением, если $m<\infty)$. Число ind $\mathscr{A}=\operatorname{dim} \operatorname{Ker} \mathscr{A}-\operatorname{codim} \operatorname{Im} \mathscr{A}$, где $\operatorname{codim} \operatorname{Im} \mathscr{A}=$ $\operatorname{dim}(\mathscr{Y} / \operatorname{Im} \mathscr{A})$, называется индексом фредгольмова (полуфредгольмова) отношения $\mathscr{A}$.

ОПРЕДЕЛЕНИЕ 1.3. Пара замкнутых подпространств $E, F$ из $\mathscr{X}$ называется регулярной, если $\mathscr{X}$ является их прямой суммой: $\mathscr{X}=E \oplus F$.

ОПРЕДЕЛЕНИЕ 1.4. Пусть $E, F$ - замкнутые подпространства из банахова пространства $\mathscr{X}$. Рассмотрим следующие условия:

1) $E \cap F=\{0\}$;

2) $1 \leqslant n=\operatorname{dim}(E \cap F) \leqslant \infty$;

3) $E \cap F$ - дополняемое подпространство в $\mathscr{X}$;

4) $E+F$ - замкнутое подпространство в $\mathscr{X}$, что эквивалентно положительности величины

$$
\gamma(E, F)=\inf _{x \in E, x \in F} \frac{\operatorname{dist}(x, F)}{\operatorname{dist}(x, E \cap F)} \quad(\leqslant 1),
$$

если $E$ не содержится в $F$;

5) $E \cap F=\{0\}$ и $\gamma(E, F)>0$ (корректная пара подпространств);

6) $E+F$ - замкнутое дополняемое в $\mathscr{X}$ подпространство;

7) $E+F-$ замкнутое подпространство из $\mathscr{X}$ коразмерности $1 \leqslant m \leqslant \infty$;

8) $E+F=\mathscr{X}$;

9) $\mathscr{X}=E \oplus F$.

Если для пары подпространств $E, F$ из $\mathscr{X}$ выполнены все условия из совокупности условий $S_{0}=\left\{i_{1}, \ldots, i_{k}\right\}$, где $1 \leqslant i_{1}<i_{2}<\cdots<i_{k} \leqslant 9$, то будем говорить, что пара подпространств $E, F$ находится в состоянии $S_{0}$. Множество состояний пары $E, F$ обозначим символом $\operatorname{St}_{\mathrm{reg}}(E, F)$.

ОПРЕДЕЛЕНИЕ 1.5. Если пара замкнутых подпространств $E, F$ из банахова пространства $\mathscr{X}$ находится в одном из состояний $\{1,7\},\{2,7\},\{2,8\}$, где $n, m<\infty$ (в частности, величина (1.4) положительна), то пара $E, F$ называется фредгольмовой. Если пара $E, F$ находится в одном из состояний $\{1,7\}$, $\{2,7\},\{2,8\}$, где только одно из чисел $n, m$ бесконечно, то она называется полуфредгольмовой. Число $\operatorname{ind}(E, F)=\operatorname{dim}(E \cap F)-\operatorname{codim}(E+F)$ называется индексом фредгольмовой (полуфредгольмовой) пары $E, F$.

ЗАмечание 1.1. Равенство $\operatorname{St}_{\text {inv }}(\mathscr{A})=\operatorname{St}_{\text {inv }}(\mathscr{B})$ для $\mathscr{A} \in \operatorname{LRC}\left(\mathscr{X}_{1}, \mathscr{Y}_{1}\right), \mathscr{B} \in$ $\operatorname{LRC}\left(\mathscr{X}_{2}, \mathscr{Y}_{2}\right)$, где $\mathscr{X}_{k}, \mathscr{Y}_{k}, k=1,2,-$ банаховы пространства, будет означать (в формулировке приводимых утверждений), что каждое из указанных девяти свойств определения 1.1 выполнено или не выполнено одновременно для $\mathscr{A}$ и $\mathscr{B}$. Равенство $\operatorname{St}_{\text {inv }}(\mathscr{A})=\operatorname{St}_{\text {reg }}(E, F)$ для $\mathscr{A} \in L R\left(\mathscr{X}_{1}, \mathscr{X}_{2}\right)$ и пары $E, F$ замкнутых подпространств из банахова пространства $\mathscr{X}$ означает, что если некоторое 
свойство из определения 1.1 выполнено для $\mathscr{A}$, то свойство с тем же порядковым номером из определения 1.4 выполнено и для пары подпространств $E, F$. Верно и обратное.

Определение и исследование дифференциальных и разностных операторов осуществляется с использованием семейства эволюционных операторов, причем не обязательно соответствующих дифференциальному уравнению вида (1.1).

Пусть $\mathbb{J}$ - некоторое подмножество в $\mathbb{R}$ с топологией, индуцируемой из $\mathbb{R}$, и $\Delta_{\mathbb{J}}=\left\{(t, s) \in \mathbb{J}^{2}: s \leqslant t\right\} \subset \mathbb{R}^{2}$. Отображение $\mathscr{U}: \Delta_{\mathbb{J}} \rightarrow$ End $X$ называется (сильно непрерывным и экспоненциально ограниченным) семейством эволюционных операторов ("вперед") на $\mathbb{J}$, если выполнены следующие условия:

1) $\mathscr{U}(t, t)=I$ - тождественный оператор для любого $t \in \mathbb{J}$;

2) $\mathscr{U}(t, s) \mathscr{U}(s, \tau)=\mathscr{U}(t, \tau), \tau \leqslant s \leqslant t, s, t, \tau \in \mathbb{J}$;

3) отображение $(t, s) \mapsto \mathscr{U}(t, s) x: \Delta_{\mathbb{J}} \rightarrow X$ непрерывно для любого $x \in X$;

4) существуют постоянные $M \geqslant 1$ и $\alpha \in \mathbb{R}$ такие, что

$$
\|\mathscr{U}(t, s)\| \leqslant M e^{\alpha(t-s)}, \quad s \leqslant t, \quad s, t \in \mathbb{J} .
$$

Семейство $\mathscr{U}: \Delta_{\mathbb{J}} \rightarrow$ End $X$ называется семейством эволюиионных операторов ("назад"), если для него выполнены условия 1), 3), 4) из приведенного определения, а условие 2) заменяется на условие

$\left.2^{\prime}\right) \mathscr{U}(s, \tau) \mathscr{U}(\tau, t)=\mathscr{U}(s, t)$ для всех $s \leqslant \tau \leqslant t$ из $\mathbb{J}$.

Если $\mathbb{J} \subset \mathbb{Z}$, то семейство $\mathscr{U}: \Delta_{\mathbb{J}} \rightarrow$ End $X$ назовем дискретным. Если не оговорено противное, в дальнейшем рассматриваются семейства эволюционных операторов "вперед".

Мы полагаем $\mathbb{R}_{-, a}=(-\infty, a], \mathbb{R}_{a,+}=[a, \infty), \mathbb{R}_{-}=(-\infty, 0], \mathbb{R}_{+}=[0, \infty)$.

Эволюционные семейства операторов естественным образом появляются в связи с представлением решений абстрактной задачи Коши

$$
x(s)=x_{0} \in D(A(s)), \quad t \geqslant s, \quad t, s \in \mathbb{J},
$$

для линейного дифференциального уравнения (1.2), где $\mathbb{J}$ - одно из множеств $[a, b], \mathbb{R}_{-, a}, \mathbb{R}_{a,+}, \mathbb{R}$.

Будем говорить, что семейство эволюционных операторов $\mathscr{U}: \Delta_{\mathbb{J}} \rightarrow \operatorname{End} X$ решает абстрактную задачу Коши (1.2), (1.5), если для любого $s \in \mathbb{J}$ существует плотное в $X$ подпространство $X_{s}$ из $D(A(s))$ такое, что для каждого $x_{0} \in X_{s}$ функция $x(t)=\mathscr{U}(t, s) x_{0}, t \in \mathbb{J}$, при всех $t \geqslant s$ дифференцируема, $x(t) \in D(A(t))$ для любого $t \in \mathbb{J}$ и выполнены равенства (1.5), (1.2). В этом случае будем также говорить, что семейство $\mathscr{U}$ соответствует задаче (1.5), (1.2).

Если функция $f: \mathbb{J} \rightarrow X$ принадлежит линейному пространству $L_{\text {loc }}^{1}(\mathbb{J}, X)$ локально суммируемых измеримых по Бохнеру (классов) функций, определенных на промежутке $\mathbb{J}$ и принимающих значения в $X$, то (слабым) решением уравнения (1.1) (при условии, что семейство $\mathscr{U}$ на $\mathbb{J}$ решает задачу Коши $(1.5),(1.2))$ называется любая непрерывная функция $x: \mathbb{J} \rightarrow X$, для которой при всех $s \leqslant t$ из $\mathbb{J}$ выполнено равенство

$$
x(t)=\mathscr{U}(t, s) x(s)-\int_{s}^{t} \mathscr{U}(t, \tau) f(\tau) d \tau .
$$


Особо отметим, что рассматриваемые в статье линейные операторы строятся по произвольному эволюционному семейству операторов, но для них тем не менее применяется термин "дифференциальный оператор".

Каждому семейству эволюционных операторов $\mathscr{U}: \Delta_{\mathbb{J}} \rightarrow \operatorname{End} X$, где $\mathbb{J}-$ промежуток из $\mathbb{R}$, можно поставить в соответствие линейный оператор

$$
\mathscr{L}_{\text {max }}=\mathscr{L}_{\text {max }, \mathbb{J}}: D\left(\mathscr{L}_{\max }\right) \subset L_{\text {loc }}^{1}(\mathbb{J}, X) \rightarrow L_{\text {loc }}^{1}(\mathbb{J}, X),
$$

который определяется следующим образом. Непрерывная функция $x: \mathbb{J} \rightarrow X$ включается в $D\left(\mathscr{L}_{\max }\right)$, если существует функция $f \in L_{\text {loc }}^{1}(\mathbb{J}, X)$ такая, что для всех $s \leqslant t$ из $\mathbb{J}$ верно равенство (1.6). Функция $f$ определяется однозначно, и, следовательно, определение оператора $\mathscr{L}_{\max }$ корректно (корректность определения оператора $\mathscr{L}_{\max }$ следует из того факта, что множество точек из $\mathbb{J}$, не являющихся точками Лебега суммируемой функции, имеет меру нуль).

Пусть $\mathscr{F}=\mathscr{F}(\mathbb{J}, X)$ - однородное пространство функций (см. раздел 2$)$. Однородными пространствами, в частности, являются определяемые в разделе 2 банаховы пространства

$$
L^{p}(\mathbb{J}, X), \quad p \in[1, \infty] ; \quad S^{p}(\mathbb{J}, X), \quad p \in[1, \infty] ; \quad C_{b}(\mathbb{J}, X) .
$$

В дальнейшем через $E$ обозначается замкнутое линейное подпространство из $X$.

Пусть $a \in \mathbb{R}$ и $\mathbb{J} \in\left\{\mathbb{R}_{-, a}, \mathbb{R}_{a,+}, \mathbb{R}\right\}$. В данной статье исследуются операторы

$$
\begin{aligned}
& \mathscr{L}=\mathscr{L}_{\mathscr{U}}: D(\mathscr{L}) \subset \mathscr{F}(\mathbb{R}, X) \rightarrow \mathscr{F}(\mathbb{R}, X), \\
& \mathscr{L}_{E}^{a,+}: D\left(\mathscr{L}_{E}^{a,+}\right) \subset \mathscr{F}\left(\mathbb{R}_{a,+}, X\right) \rightarrow \mathscr{F}\left(\mathbb{R}_{a,+}, X\right), \\
& \mathscr{L}_{E}^{-, a}: D\left(\mathscr{L}_{E}^{-, a}\right) \subset \mathscr{F}\left(\mathbb{R}_{-, a}, X\right) \rightarrow \mathscr{F}\left(\mathbb{R}_{-, a}, X\right),
\end{aligned}
$$

области определения которых имеют вид

$$
\begin{array}{r}
D(\mathscr{L})=\left\{x \in D\left(\mathscr{L}_{\max , \mathbb{R}}\right) \cap \mathscr{F}(\mathbb{R}, X) \mid \mathscr{L}_{\max } x \in \mathscr{F}(\mathbb{R}, X)\right\}, \\
D\left(\mathscr{L}_{E}^{a,+}\right)=\left\{x \in D\left(\mathscr{L}_{\max , \mathbb{R}}\right) \cap \mathscr{F}\left(\mathbb{R}_{a,+}, X\right) \mid x(a) \in E,\right. \\
\left.\mathscr{L}_{\max , \mathbb{R}_{a,+}} x \in \mathscr{F}\left(\mathbb{R}_{a,+}, X\right)\right\}, \\
D\left(\mathscr{L}_{E}^{-, a}\right)=\left\{x \in D\left(\mathscr{L}_{\max , \mathbb{R}_{-, a}}\right) \cap \mathscr{F}\left(\mathbb{R}_{-, a}, X\right) \mid x(a) \in E,\right. \\
\left.\mathscr{L}_{\max , \mathbb{R}_{-, a}} x \in \mathscr{F}\left(\mathbb{R}_{-, a}, X\right)\right\}
\end{array}
$$

соответственно. При этом, если $x$ принадлежит области определения соответствующего оператора, то его значение на функции $x$ полагается равным $f=\mathscr{L}_{\max , \rrbracket} x$, т. е. пара $(x, f)$ удовлетворяет равенству $(1.6)$. Если $a=0$, то положим

$$
\mathscr{L}_{E}^{+}=\mathscr{L}_{E}^{a,+}, \quad \mathscr{L}_{E}^{-}=\mathscr{L}_{E}^{-, a} .
$$

Отметим, что операторы $\mathscr{L}, \mathscr{L}_{E}^{-, a}$ замкнуты, а оператор $\mathscr{L}_{E}^{a,+}$ может быть незамкнутым (см. [19; пример 5.1]). 
Если семейство $\mathscr{U}$ решает задачу Коши (1.2), (1.5), то иногда для введенных в рассмотрение операторов будет использоваться запись $-d / d t+A(t)$.

Пусть $\mathbb{J} \in\left\{\mathbb{R}_{-}, \mathbb{R}\right\}$. Тогда в банаховом пространстве $\mathscr{F}=\mathscr{F}(\mathbb{J}, X)$ корректно определена полугруппа $T: \mathbb{R}_{+} \rightarrow$ End $\mathscr{F}$ операторов взвешенного сдвига вида

$$
(T(t) x)(s)=\mathscr{U}(s, s-t) x(s-t), \quad s \in \mathbb{J}, \quad x \in \mathscr{F}, \quad t \geqslant 0 .
$$

В случае когда $\mathbb{J}=\mathbb{R}$, а $X$ - гильбертово пространство, такая полугруппа введена в рассмотрение Дж. С. Хоулэндом [20] в гильбертовом пространстве $L^{2}(\mathbb{R}, X)$ при условии, что операторы $\mathscr{U}(t, s), t, s, \in \mathbb{R}$, унитарны. Следующие две теоремы позволяют использовать теорию полугрупп операторов, а также разностные операторы.

Tеорема 1.1 [13], [14]. Если $\mathscr{F}(\mathbb{R}, X) \in\left\{L^{p}(\mathbb{R}, X), p \in[1, \infty) ; C_{0}(\mathbb{R}, X)\right\}$, то полугруппа операторов (1.7) сильно непрерьвна, а ее генератором являетcя оператор $\mathscr{L}=\mathscr{L}_{\mathscr{U}}$.

Tеорема 1.2. Спектры операторов $T(t) \in$ End $\mathscr{F}(\mathbb{R}, X), t \in \mathbb{R}_{+}$, связаны соотношением

$$
\sigma(T(t)) \backslash\{0\}=\exp \sigma(\mathscr{L}) t=\{\exp (\lambda t) ; \lambda \in \sigma(\mathscr{L})\} .
$$

Для $\mathscr{F}(\mathbb{R}, X) \in\left\{L^{p}(\mathbb{R}, X), p \in[1, \infty) ; C_{0}(\mathbb{R}, X)\right\}$ теорема 1.2 была доказана в [13], [14], [16], [17], при этом в [13], [14] рассматривались более общие пространства. Здесь и далее символ $C_{0}(\mathbb{J}, X)$ обозначает подпространство функций $x$ из $C_{b}(\mathbb{J}, X)$, обладающих свойством $\lim _{|t| \rightarrow \infty}\|x(t)\|=0$. Для оператора $\mathscr{L}_{E}^{+}$ прямого аналога теоремы 1.1 нет, но в статье [19] введена в рассмотрение полугруппа разностных отношений на банаховом пространстве $\mathscr{F}\left(\mathbb{R}_{+}, X\right)$ и получен аналог теоремы 1.2 .

Применение разностных операторов из (1.7) и разностных отношений на пространстве $\mathscr{F}(\mathbb{J}, X)$ для исследования операторов $\mathscr{L}$ и $\mathscr{L}_{E}^{+}$вызывает затруднение в связи с проблемой использования сопряженного пространства к $\mathscr{F}(\mathbb{J}, X)$. Кроме того, для операторов $\mathscr{L}$ и $\mathscr{L}_{E}^{+}$трудно использовать спектральную теорию операторов из-за неограниченности спектральных компонент.

В данной статье изучение рассматриваемых дифференциальных операторов проводится с существенным привлечением разностных операторов и отношений на однородных пространствах векторных последовательностей. Такие операторы и отношения, как правило, имеют ограниченные спектральные компоненты, и поэтому появляется возможность построения проекторов Рисса по спектральной компоненте. Можно сказать, что при исследовании дифференциальных уравнений, рассматриваемых на бесконечном промежутке, разностные операторы и отношения играют ту же роль, что и оператор монодромии при изучении дифференциальных уравнений с периодическими коэффициентами.

Понятие однородного пространства последовательностей $\mathscr{F}\left(\mathbb{J}_{d}, X\right)$ вводится в разделе 2 .

Пусть $\mathbb{J}_{d}$ - одно из подмножеств множества целых чисел вида

$$
\begin{array}{cl}
\mathbb{Z}, & \mathbb{Z}_{-, m}=(-\infty, m] \cap \mathbb{Z}, \quad \mathbb{Z}_{m,+}=[m, \infty) \cap \mathbb{Z}, \quad m \in \mathbb{Z}, \\
\mathbb{Z}_{-}=\{n \in \mathbb{Z} \mid n \leqslant 0\}, \quad \mathbb{Z}_{+}=\{n \in \mathbb{Z} \mid n \geqslant 0\} .
\end{array}
$$


Одним из однородных пространств является банахово пространство $\ell^{p}\left(\mathbb{J}_{d}, X\right)$, $p \in[1, \infty]$, последовательностей $x: \mathbb{J}_{d} \rightarrow X$, для которых конечна величина (принимаемая за норму)

$$
\|x\|_{p}=\left(\sum_{k \in \mathbb{J}_{d}}\|x(k)\|^{p}\right)^{1 / p}, \quad p \in[1, \infty) ; \quad\|x\|_{\infty}=\sup _{k \in \mathbb{J}_{d}}\|x(k)\|, \quad p=\infty .
$$

Последовательность $x: \mathbb{N} \rightarrow X$ будет иногда обозначаться символом $\left(x_{n}\right)$.

Пусть $U: \mathbb{J}_{d} \rightarrow$ End $X$ - ограниченная функция и $E$ - замкнутое подпространство банахова пространства $X$.

Изучаются линейные разностные операторы

$$
\mathscr{D} \in \operatorname{End} \mathscr{F}(\mathbb{Z}, X), \quad \mathscr{D}_{E}^{-, a}: D\left(\mathscr{D}_{E}^{-, a}\right) \subset \mathscr{F}\left(\mathbb{Z}_{-, a}, X\right) \rightarrow \mathscr{F}\left(\mathbb{Z}_{-, a}, X\right), \quad a \in \mathbb{Z},
$$

и разностные отношения $\mathscr{D}_{E}^{a,+} \in \operatorname{LRC}\left(\mathscr{F}\left(\mathbb{Z}_{a,+}, X\right)\right), a \in \mathbb{Z}$, которые определяются равенствами

$$
\begin{aligned}
& (\mathscr{D} x)(n)=x(n)-U(n) x(n-1), \quad n \in \mathbb{Z}, \quad x \in \mathscr{F}(\mathbb{Z}, X), \\
& \left(\mathscr{D}_{E}^{-, a} x\right)(n)=x(n)-U(n) x(n-1), \quad n \leqslant a, \quad x \in D\left(\mathscr{D}_{E}^{-, a}\right),
\end{aligned}
$$

где $D\left(\mathscr{D}_{E}^{-, a}\right)=\left\{x \in \mathscr{F}\left(\mathbb{Z}_{-, a}, X\right) \mid x(a) \in E\right\}$,

$$
\begin{gathered}
\left(\mathscr{D}_{E}^{a,+}\right)=\left\{(x, y) \in \mathscr{F}\left(\mathbb{Z}_{a,+}, X\right) \mid y(n)=x(n)-U(n) x(n-1), n \geqslant a+1,\right. \\
\left.y(a)=x(a)+x_{0} \text { для некоторого } x_{0} \in E\right\} .
\end{gathered}
$$

Если $a=0$, то положим $\mathscr{D}_{E}^{-}=\mathscr{D}_{E}^{-, a}, \mathscr{D}_{E}^{+}=\mathscr{D}_{E}^{a,+}$. Отметим, что для определения разностного отношения $\mathscr{D}_{E}^{a,+}$ функцию $U$ можно считать определенной на $\mathbb{Z}_{a+1,+}$.

При изучении операторов $\mathscr{D}, \mathscr{D}_{E}^{-, a}$ и отношения $\mathscr{D}_{E}^{a,+}$ используются соответствующие дискретные семейства эволюционных операторов $\mathscr{U}=\mathscr{U}_{d}=\mathscr{U}_{\mathbb{J}_{d}}$ : $\Delta_{\mathbb{J}_{d}} \rightarrow$ End $X$ вида

$$
\mathscr{U}_{d}(n, m)=\mathscr{U}_{\mathbb{J}_{d}}(n, m)= \begin{cases}U(n) U(n-1) \cdots U(m+1), & m<n, \\ I, & m=n,\end{cases}
$$

где $m, n \in \mathbb{J}_{d} \in\left\{\mathbb{Z}, \mathbb{Z}_{-, a}, \mathbb{Z}_{a,+}\right\}$. Результаты для разностных операторов и отношений получены без предположения, что семейство $\mathscr{U}_{d}$ порождается семейством эволюционных операторов $\mathscr{U}: \Delta_{\mathbb{J}} \rightarrow$ End $X$, где $\mathbb{J} \in\left\{\mathbb{R}_{-}, \mathbb{R}_{+}, \mathbb{R}\right\}$.

Исследование свойств из определения 1.1 для рассматриваемых дифференциальных операторов и разностных операторов (отношений) ведется с привлечением понятия экспоненциальной дихотомии семейства эволюционных операторов.

ОПРЕДЕЛЕНИЕ 1.6. Пусть $\mathbb{J}$ - подмножество в $\mathbb{R}$ с топологией, индуцируемой из $\mathbb{R}$. Будем говорить, что семейство эволюционных операторов $\mathscr{U}: \Delta_{\mathbb{J}} \rightarrow$ End $X$ допускает экспоненциальную дихотомию на множестве $\Omega$ из $\mathbb{J}$, если существуют ограниченная сильно непрерывная проекторнозначная функция 
$P: \Omega \rightarrow$ End $X$ и постоянные $M_{0} \geqslant 1, \gamma>0$ такие, что выполнены следующие свойства:

1) $\mathscr{U}(t, s) P(s)=P(t) \mathscr{U}(t, s), t \geqslant s, t, s \in \Omega$;

2) $\|\mathscr{U}(t, s) P(s)\| \leqslant M_{0} \exp (-\gamma(t-s))$ для $s \leqslant t, s, t \in \Omega$;

$3)$ для $s \leqslant t, s, t \in \Omega$, сужение $\mathscr{U}_{t, s}: X(s) \rightarrow X(t)$ оператора $\mathscr{U}(t, s)$ на область значений $X(s)=\operatorname{Im} Q(s)$ дополнительного к $P(s)$ проектора $Q(s)=$ $I-P(s)$ есть изоморфизм подпространств $X^{\prime}(s)$ и $X^{\prime}(t)=\operatorname{Im} Q(t)$ (мы полагаем оператор $\mathscr{U}(s, t) \in$ End $X$ равным $\mathscr{U}_{t, s}^{-1}$ на $X^{\prime}(t)$ и равным нулевому оператору на $X(t)=\operatorname{Im} P(t) \subset \mathscr{X})$;

4) $\|\mathscr{U}(s, t)\| \leqslant M_{0} \exp \gamma(s-t)$ для всех $t \geqslant s$ из $\Omega$.

Пару проекторнозначных функций, участвующих в определении 1.6, назовем расщепляющей парой для семейства $\mathscr{U}$. Если $P=0$ или $Q=0$, то будем говорить, что для $\mathscr{U}$ имеет место тривиальная экспоненииальная дихотомия на $\Omega$.

Аналогичное определение экспоненциальной дихотомии дается и в случае, когда $\mathscr{U}$ - семейство эволюционных операторов "назад".

Teорема 1.3. Пусть $\mathscr{L}_{E}^{+}: D\left(\mathscr{L}_{E}^{+}\right) \subset \mathscr{F}\left(\mathbb{R}_{+}, X\right) \rightarrow \mathscr{F}\left(\mathbb{R}_{+}, X\right)-$ замкнутый линейный оператор, действующий в однородном пространстве функицй $\mathscr{F}\left(\mathbb{R}_{+}, X\right)$. Тогда

$$
\operatorname{St}_{\text {inv }}\left(\mathscr{L}_{E}^{+}\right)=\operatorname{St}_{\text {inv }}\left(\mathscr{D}_{E}^{+}\right)
$$

где отношение $\mathscr{D}_{E}^{+}$рассматривается на ассоциированном с $\mathscr{F}\left(\mathbb{R}_{+}, X\right)$ однородном пространстве последовательностей $\mathscr{F}\left(\mathbb{Z}_{+}, X\right)$ (см. замечание 2.1$)$. $B$ частности, $\mathscr{L}_{E}^{+}$и $\mathscr{D}_{E}^{+}$одновременно фредгольмовы (полуфредголъмовы) и при условии фредгольмовости (полуфредгольмовости) одного из них верны равенства

$$
\begin{gathered}
\operatorname{dim} \operatorname{Ker} \mathscr{L}_{E}^{+}=\operatorname{dim} \operatorname{Ker} \mathscr{D}_{E}^{+}, \quad \operatorname{codim} \operatorname{Im} \mathscr{L}_{E}^{+}=\operatorname{codim} \operatorname{Im} \mathscr{D}_{E}^{+}, \\
\text {ind } \mathscr{L}_{E}^{+}=\operatorname{ind} \mathscr{D}_{E}^{+} .
\end{gathered}
$$

Эта теорема доказывается в конце раздела 4. При этом в замечании 4.1 для оператора $\mathscr{L}_{E}^{+}$уточняется содержание свойства 3$)$ из определения 1.1. Следующая теорема является непосредственным следствием теорем 1.3, 1.7-1.9.

Теорема 1.4. Свойство фредголъмовости оператора $\mathscr{L}_{E}^{+}$и его индекс не зависят от выбора однородного пространства $\mathscr{F}\left(\mathbb{R}_{+}, X\right)$.

Включение $\operatorname{St}_{\text {inv }}\left(\mathscr{L}_{E}^{+}\right) \subset \operatorname{St}_{\text {inv }}\left(\mathscr{D}_{E}^{+}\right)$было установлено в [19; теорема 5.8]. Kроме того, в [19] было доказано, что любое из девяти свойств в определении 1.1, выполненное для $\mathscr{D}_{E}^{+}$, исключая, быть может, свойство 3) о дополняемости ядра, выполнено и для $\mathscr{L}_{E}^{+}$.

Аналоги теорем 1.3 и 1.4 верны и для операторов $\mathscr{L}_{E}^{-}$и $\mathscr{D}_{E}^{-}$.

Tеорема 1.5. Для линейного оператора $\mathscr{L}: D(\mathscr{L}) \subset \mathscr{F}(\mathbb{R}, X) \rightarrow \mathscr{F}(\mathbb{R}, X)$, действующего в однородном пространстве $\mathscr{F}(\mathbb{R}, X)$, и разностного оператора $\mathscr{D} \in$ End $\mathscr{F}(\mathbb{Z}, X)$, действующего в ассоииированном с $\mathscr{F}(\mathbb{R}, X)$ однородном пространстве последовательностей $\mathscr{F}(\mathbb{Z}, X)$, имеет место равенство их множеств состояний

$$
\operatorname{St}_{\text {inv }}(\mathscr{L})=\operatorname{St}_{\text {inv }}(\mathscr{D})
$$


$B$ частности, операторы $\mathscr{L}$ и $\mathscr{D}$ одновременно фредгольмовы (полуфредгольмови) и при условии фредгольмовости (полуфредгольмовости) одного из них вернь равенства

$\operatorname{dim} \operatorname{Ker} \mathscr{L}=\operatorname{dim} \operatorname{Ker} \mathscr{D}, \quad \operatorname{codim} \operatorname{Im} \mathscr{L}=\operatorname{codim} \operatorname{Im} \mathscr{D}, \quad$ ind $\mathscr{L}=$ ind $\mathscr{D}$.

Теорема 1.6. Свойство фредгольмовости оператора $\mathscr{L}$ и его индекс не зависят от выбора однородного пространства $\mathscr{F}(\mathbb{R}, X)$.

Отметим, что теорема 1.6 непосредственно следует из теорем $1.5,1.13,1.15$, 1.16 .

ОПРЕДЕЛЕНИЕ 1.7. Пусть подмножество $\mathbb{J}$ из $\mathbb{R}$ содержит последовательность $\left(t_{n}\right)$ со свойством $\lim _{n \rightarrow \infty} t_{n}=\infty$ (со свойством $\left.\lim _{n \rightarrow \infty} t_{n}=-\infty\right)$. Будем говорить, что семейство эволюционных операторов $\mathscr{U}: \Delta_{\mathbb{J}} \rightarrow \operatorname{End} X$ несингулярно на $+\infty$ (соответственно на $-\infty$ ), если существует такое число $a \in \mathbb{J}$, что семейство $\mathscr{U}$ допускает экспоненциальную дихотомию на множестве $\mathbb{J} \cap[a, \infty)$ (соответственно на множестве $\mathbb{J} \cap(-\infty, a])$.

Теорема 1.7. Пусть семейство $\mathscr{U}: \Delta_{\mathbb{Z}_{+}} \rightarrow$ End $X$ допускает экспоненииальную дихотомию на множестве $\mathbb{Z}_{a,+}$, әде $a \in \mathbb{Z}_{+}$, с расщепляющей парой проекторнозначных функиий $P_{+}, Q_{+}: \mathbb{Z}_{a,+} \rightarrow$ End $X$. Тогда

$$
\operatorname{St}_{\text {inv }}\left(\mathscr{D}_{E}^{+}\right)=\operatorname{St}_{\text {inv }}\left(\mathscr{N}_{a}^{+}\right),
$$

где оператор $\mathscr{N}_{a}^{+}: E \rightarrow \operatorname{Im} Q_{+}(a)$ определен формулой

$$
\mathscr{N}_{a}^{+} x=Q_{+}(a) \mathscr{U}(a, 0) x, \quad x \in E .
$$

ТЕОрема 1.8. Пусть семейство эволюиионных операторов $\mathscr{U}: \Delta_{\mathbb{R}_{a,+}} \rightarrow$ End $X$ допускает экспоненииальную дихотомию на множестве $\mathbb{R}_{a,+}$, где $a \in \mathbb{R}_{+}$, с расщепляющей парой проекторнозначнъх функций $P_{+}, Q_{+}: \mathbb{R}_{a,+} \rightarrow$ End $X$. Tогдa

$$
\operatorname{St}_{\text {inv }}\left(\mathscr{L}_{E}^{+}\right)=\operatorname{St}_{\text {inv }}\left(\mathscr{N}_{a}^{+}\right)
$$

где оператор $\mathscr{N}_{a}^{+}: E \rightarrow \operatorname{Im} Q_{+}($a) определен формулой (1.17).

Tеорема 1.9. Следующие условия эквивалентнъ:

1) оператор $\mathscr{L}_{E}^{+}: D\left(\mathscr{L}_{E}^{+}\right) \subset \mathscr{F}\left(\mathbb{R}_{+}, X\right) \rightarrow \mathscr{F}\left(\mathbb{R}_{+}, X\right)$ фредгольмов;

2) существует число $a \in \mathbb{R}_{+}$такое, что семейство $\mathscr{U}$ допускает экспоненииальную дихотомию на $[a, \infty)$ с такой расщепляющей парой проекторнозначнъцх функиий $P_{+}, Q_{+}:[a, \infty) \rightarrow$ End $X$, что оператор $\mathscr{N}_{a}^{+}: E \rightarrow \operatorname{Im} Q_{+}(a)$, определенный формулой (1.17), является фредгольмовым.

Если выполнено условие 2), то имеют место равенства

$$
\begin{gathered}
\operatorname{dim} \operatorname{Ker} \mathscr{L}_{E}^{+}=\operatorname{dim} \operatorname{Ker} \mathscr{N}_{a}^{+}, \quad \operatorname{codim} \operatorname{Im} \mathscr{L}_{E}^{+}=\operatorname{codim} \operatorname{Im} \mathscr{N}_{a}^{+}, \\
\text {ind } \mathscr{L}_{E}^{+}=\operatorname{ind} \mathscr{N}_{a}^{+} .
\end{gathered}
$$


ТЕОрема 1.10. Пусть все операторы $\mathscr{U}(t, s), s<t, s, t \in \mathbb{R}_{+}$, компактны и семейство эволюиионных операторов $\mathscr{U}: \Delta_{\mathbb{R}_{+}} \rightarrow$ End $X$ несингулярно на $+\infty$. Тогда следуюшие условия эквивалентны:

1) оператор $\mathscr{L}_{E}^{+}: D\left(\mathscr{L}_{E}^{+}\right) \subset \mathscr{F}\left(\mathbb{R}_{+}, X\right) \rightarrow \mathscr{F}\left(\mathbb{R}_{+}, X\right)$ фредгольмов;

2) отношение $\mathscr{D}_{E}^{+} \in \operatorname{LRC}\left(\mathscr{F}\left(\mathbb{Z}_{+}, X\right)\right)$ фредгольмово;

3) $E$ - конечномерное подпространство из $X$.

Теорема 1.11. Пусть семейство эволюиионнъх операторов $\mathscr{U}: \Delta_{\mathbb{R}_{+}} \rightarrow X$ допускает экспоненциальную дихотомию на $\mathbb{R}_{+}$с расщепляющей парой проекторнозначных функций $P, Q: \mathbb{R}_{+} \rightarrow$ End $X$. Тогда

$$
\operatorname{St}_{\text {inv }}\left(\mathscr{L}_{E}^{+}\right)=\operatorname{St}_{\text {inv }}\left(\mathscr{D}_{E}^{+}\right)=\operatorname{St}_{\text {reg }}(E, \operatorname{Im} P(0)) .
$$

Важно отметить, что если семейство эволюционных операторов $\mathscr{U}: \Delta_{\mathbb{R}_{+}} \rightarrow$ End $X$ строится для дифференциального уравнения (1.2) с функцией $A$, принадлежащей $S^{1}\left(\mathbb{R}_{+}\right.$, End $\left.X\right)$, то из его несингулярности на $+\infty$ следует, что $\mathscr{U}$ допускает экспоненциальную дихотомию на $\mathbb{R}_{+}$. Однако это свойство теряется для более общих семейств эволюционных операторов. Если оператор $\mathscr{L}_{E}^{+}$ фредгольмов, то семейство $\mathscr{U}$ несингулярно на $+\infty$, но $\mathscr{U}$ может не обладать свойством экспоненциальной дихотомии на $\mathbb{R}_{+}$(на $\mathbb{Z}_{+}$; см. пример 8.6).

Многие утверждения настоящей статьи для операторов $\mathscr{D} \in \operatorname{End} \mathscr{F}(\mathbb{Z}, X)$, $\mathscr{L}: D(\mathscr{L}) \subset \mathscr{F}(\mathbb{R}, X) \rightarrow \mathscr{F}(\mathbb{R}, X)$ получены при условии, что выполнено следующее предположение.

ПреДПОЛОЖеНИЕ 1.1. Пусть $\mathbb{J} \in\{\mathbb{Z}, \mathbb{R}\}$. Существуют иисла $a, b \in \mathbb{J}$, $a \leqslant b$, такие, что семейство эволюиионных операторов $\mathscr{U}: \Delta_{\mathbb{J}} \rightarrow$ End $X$ допускает экспоненциальную дихотомию на множествах $\mathbb{J}_{-, a}=\{t \in \mathbb{J} \mid t \leqslant a\}$, $\mathbb{J}_{b,+}=\{t \in \mathbb{J} \mid t \geqslant b\}$ с расщепляющими парами проекторнозначных функиий $P_{-}, Q_{-}: \mathbb{J}_{-, a} \rightarrow$ End $X, P_{+}, Q_{+}: \mathbb{J}_{b,+} \rightarrow$ End $X$.

Важные результаты об операторе $\mathscr{L}$ были получены В. В. Жиковым в статье [8], где была доказана эквивалентность условия обратимости оператора $\mathscr{L}$ в пространстве $C_{b}(\mathbb{R}, X)$ условию экспоненциальной дихотомии семейства $\mathscr{U}$. Затем эти результаты были изложены в монографии [9]. Кроме того, отметим, что в [8], [9] систематическим стало использование теории операторов в исследовании качественных свойств решений дифференциальных уравнений в банаховом пространстве.

Из формулировки приведенных утверждений следует, что исследование рассматриваемых дифференциальных и разностных операторов (уравнений) ведется с существенным использованием понятия экспоненциальной дихотомии семейства эволюционных операторов, по которому определяется изучаемый оператор. Свойство экспоненциальной дихотомии семейства эволюционных операторов наиболее естественно выявляется при рассмотрении разностного непрерывно обратимого оператора $\mathscr{D}=\mathscr{D}_{\mathscr{U}}$, определенного формулой (1.8). В этом случае спектр $\sigma(\mathscr{K})$ оператора взвешенного сдвига $\mathscr{K}=I-\mathscr{D}$ не содержит точек единичной окружности $\mathbb{T}=\{\lambda \in \mathbb{C}|| \lambda \mid=1\}$. Следовательно, $\sigma(\mathscr{K})=\sigma_{\text {int }} \cup \sigma_{\text {out }}$, где $\sigma_{\text {int }}=\{\lambda \in \sigma(\mathscr{K})|| \lambda \mid 1\}$. Такое представление множества $\sigma(\mathscr{K})$ позволяет построить по спектральным множествам $\sigma_{\text {int }}$, 
$\sigma_{\text {out }}$ проекторы Рисса $\mathscr{P}_{\text {int }}, \mathscr{P}_{\text {out }}=I-\mathscr{P}_{\text {int }}$ такие, что $\sigma\left(\mathscr{K} \mid \mathscr{F}_{\text {int }}\right)=\sigma_{\text {int }}$, $\sigma\left(\mathscr{K} \mid \mathscr{F}_{\text {out }}\right)=\sigma_{\text {out }}$, где $\mathscr{F}_{\text {int }}=\operatorname{Im} \mathscr{P}_{\text {int }}, \mathscr{F}_{\text {out }}=\operatorname{Im} \mathscr{P}_{\text {out }}$. При этом устанавливается, что $\mathscr{P}_{\text {int }}, \mathscr{P}_{\text {out }}$ являются операторами умножения на ограниченные операторнозначные функции. Следовательно, $r\left(\mathscr{K}_{\text {int }}\right)<1$ (где $r(\cdot)$ - спектральный радиус и $\mathscr{K}_{\text {int }}=\mathscr{K} \mid \mathscr{F}_{\text {int }}-$ сужение оператора $\mathscr{K}$ на $\left.\mathscr{F}_{\text {int }}\right)$, оператор $\mathscr{K}_{\text {out }}=\mathscr{K} \mid \mathscr{F}_{\text {out }}$ непрерывно обратим и $r\left(\mathscr{K}_{\text {out }}^{-1}\right)<1$. Расшифровка перечисленных свойств (она подробна осуществлена в [1; теорема 6.1]) приводит к свойствам 1)-4) из определения 1.6 экспоненциальной дихотомии на множестве $\mathbb{Z}$ семейства эволюционных операторов $\mathscr{U}_{d}$, определенных равенствами (1.11). Если свойство $\mathscr{U}_{d}$ является сужением на $\Delta_{\mathbb{Z}}$ семейства эволюционных операторов $\mathscr{U}: \Delta_{\mathbb{R}} \rightarrow \operatorname{End} X$, то свойством экспоненциальной дихотомии будет обладать и $\mathscr{U}$. Итак, следующие свойства эквивалентны (см. статьи [13], [14]):

1) оператор $\mathscr{D} \in$ End $\mathscr{F}(\mathbb{Z}, X)$ непрерывно обратим;

2) оператор $\mathscr{L}=\mathscr{L}_{\mathscr{U}}: D(\mathscr{L}) \subset \mathscr{F}(\mathbb{R}, X) \rightarrow \mathscr{F}(\mathbb{R}, X)$ непрерывно обратим;

3) семейство эволюционных операторов $\mathscr{U}: \Delta_{\mathbb{R}} \rightarrow$ End $X$ допускает экспоненциальную дихотомию на $\mathbb{R}$.

Замена условия непрерывной обратимости оператора $\mathscr{D}$ (оператора $\mathscr{L}$ ) на условие его фредгольмовости влечет в силу теоремы 1.15 (теоремы 1.16) выполнение предположения 1.1 для семейства $\mathscr{U}_{d}$ (семейства $\mathscr{U}$ ). В примере 8.6 построено семейство эволюционных операторов $\mathscr{U}: \Delta_{\mathbb{R}} \rightarrow$ End $X$, обладающее свойством экспоненциальной дихотомии на $\mathbb{R}_{-}$и $[1, \infty)$, но не обладающее таким свойством на $\mathbb{R}$. В силу теорем $1.15,1.16$, при выполнении предположения 1.1 за свойство фредгольмовости операторов $\mathscr{D}_{\mathscr{U}}$ и $\mathscr{L}_{\mathscr{U}}$ "отвечает" линейный оператор

$$
\mathscr{N}_{b, a}: \operatorname{Im} Q_{-}(a) \rightarrow \operatorname{Im} Q_{+}(b), \quad \mathscr{N}_{b, a} x=Q_{+}(b) \mathscr{U}(b, a) x, \quad x \in \operatorname{Im} Q_{-}(a) .
$$

Он был определен в статьях [20], [21] и назван узловым. Важность его рассмотрения обусловлена тем, что он действует между подпространствами фазового пространства $X$ (а не в $\mathscr{F}(\mathbb{J}, X)$ ). Согласно формулируемым далее теоремам $1.12,1.13$, а также вытекающей из них теореме 1.14 , свойства операторов $\mathscr{D}$ и $\mathscr{L}$ теснейшим образом связаны со свойствами оператора $\mathscr{N}_{b, a}$ (они имеют одинаковое множество состояний).

Далее рассматриваются разностный оператор $\mathscr{D} \in \operatorname{End} \mathscr{F}(\mathbb{Z}, X)$, определенный формулой $(1.8)$, и оператор $\mathscr{L}=\mathscr{L}_{\mathscr{U}}: D(\mathscr{L}) \subset \mathscr{F}(\mathbb{R}, X) \rightarrow \mathscr{F}(\mathbb{R}, X)$. Оператор $\mathscr{D}$ строится по ограниченной функции $U: \mathbb{Z} \rightarrow$ End $X$, и далее используется семейство эволюционных операторов $\mathscr{U}=\mathscr{U}_{d}: \Delta_{\mathbb{Z}} \rightarrow \operatorname{End} X$, определенное равенствами (1.11).

ТеОрема 1.12. Пусть для семейства $\mathscr{U}=\mathscr{U}_{d}: \Delta_{\mathbb{Z}} \rightarrow$ End $X$ выполнено предположение 1.1. Тогда

$$
\operatorname{St}_{\text {inv }}(\mathscr{D})=\operatorname{St}_{\text {inv }}\left(\mathscr{N}_{b, a}\right) \text {. }
$$

$B$ частности, оператор $\mathscr{D}$ фредгольмов (полуфредгольмов) тогда и только тогда, когда фредголъмовым (полуфредголвмовым) является узловой оператор $\mathscr{N}_{b, a}$. Если оператор $\mathscr{D}$ фредгольмов, то

$$
\operatorname{dim} \operatorname{Ker} \mathscr{D}=\operatorname{dim} \operatorname{Ker} \mathscr{N}_{b, a}, \quad \operatorname{codim} \operatorname{Im} \mathscr{D}=\operatorname{codim} \operatorname{Im} \mathscr{N}_{b, a}, \quad \text { ind } \mathscr{D}=\operatorname{ind} \mathscr{N}_{b, a} .
$$


Утверждение следующей теоремы вытекает из теорем 1.5 и 1.12.

Теорема 1.13. Пусть для семейства эволюиионных операторов $\mathscr{U}: \Delta_{\mathbb{R}} \rightarrow$ End $X$ выполнено предположение 1.1. Тогда

$$
\operatorname{St}_{\text {inv }}(\mathscr{L})=\operatorname{St}_{\text {inv }}\left(\mathscr{N}_{b, a}\right)
$$

В частности, оператор $\mathscr{L}$ фредгольмов (полуфредгольмов) тогда и только тогда, когда фредголвмовым (полуфредгольмовым) является узловой оператор $\mathscr{N}_{b, a}$. Если оператор $\mathscr{L}$ фредгольмов (полуфредгольмов), то

$\operatorname{dim} \operatorname{Ker} \mathscr{L}=\operatorname{dim} \operatorname{Ker} \mathscr{N}_{b, a}, \quad \operatorname{codim} \operatorname{Im} \mathscr{L}=\operatorname{codim} \operatorname{Im} \mathscr{N}_{b, a}, \quad$ ind $\mathscr{L}=\operatorname{ind} \mathscr{N}_{b, a}$.

СледСтвиЕ 1.1. Если оператор $\mathscr{D}$ (оператор $\mathscr{L})$ фредголвмов (полуфредгольмов) в одном из однородных пространств последовательностей (функций), то он фредгольмов (полуфредгольмов) в любом однородном пространстве и его индекс (а также размерность ядра, коразмерность образа) не зависит от вида однородного пространства, где он действует.

ПредПоложение 1.2. Семейство $\mathscr{U}: \Delta_{\mathbb{J}} \rightarrow$ End $X$, где $\mathbb{J} \in\{\mathbb{Z}, \mathbb{R}\}$, допускает экспоненииальную дихотомию на множествах

$$
\mathbb{J}_{-}=\mathbb{J} \cap \mathbb{R}_{-}, \quad \mathbb{J}_{+}=\mathbb{J} \cap \mathbb{R}_{+}
$$

с расщепляющими парами проекторнозначных функиий $P_{-}, Q_{-}: \mathbb{J}_{-} \rightarrow$ End $X$, $P_{+}, Q_{+}: \mathbb{J}_{+} \rightarrow$ End $X$.

Теорема 1.14. Пусть для семейства эволющионных операторов $\mathscr{U}: \Delta_{\mathbb{R}} \rightarrow$ End $X$ выполнено предположение 1.2. Тогда

$$
\operatorname{St}_{\text {inv }}(\mathscr{L})=\operatorname{St}_{\text {reg }}\left(\operatorname{Im} Q_{-}(0), \operatorname{Im} P_{+}(0)\right) .
$$

$B$ частности, оператор $\mathscr{L}$ фредгольмов (полуфредгольмов) тогда и только тогда, когда подпространства $\operatorname{Im} P_{-}(0), \operatorname{Im} Q_{+}(0)$ образуют фредгольмову (полуфредгольмову) пару. Если оператор $\mathscr{L}$ фредгольмов (полуфредгольмов), то

$$
\begin{aligned}
\operatorname{dim} \operatorname{Ker} \mathscr{L} & =\operatorname{dim}\left(\operatorname{Im} P_{-}(0) \cap \operatorname{Im} Q_{+}(0)\right), \\
\operatorname{codim} \operatorname{Im} \mathscr{L} & =\operatorname{codim}\left(\operatorname{Im} P_{-}(0)+\operatorname{Im} Q_{+}(0)\right), \\
\text { ind } \mathscr{L} & =\operatorname{ind}\left(\operatorname{Im} P_{-}(0), \operatorname{Im} Q_{+}(0)\right) .
\end{aligned}
$$

В теореме 5.4 получен соответствующий теореме 1.14 результат для оператора $\mathscr{D}$. Равенство (1.21) позволяет заключить, что множество $\operatorname{St}_{\text {inv }}(\mathscr{L})$ не зависит от однородного пространства, где действует оператор $\mathscr{L}$. Утверждение теоремы 1.14 следует непосредственно из теоремы 1.5 и теоремы 5.4.

Первые результаты о фредгольмовости обыкновенного дифференциального оператора

$$
\mathscr{L}=-\frac{d}{d t}+A(t): C_{b}^{1}(\mathbb{R}, X) \subset C_{b}(\mathbb{R}, X) \rightarrow C_{b}(\mathbb{R}, X),
$$


где $A \in C_{b}(\mathbb{R}$, End $X), \operatorname{dim} X<\infty$, были получены Э. Мухамадиевым в [22] и его докторской диссертации [23]. Результаты о фредгольмовости оператора $\mathscr{L}$ были получены им в терминах предельных операторов и предельных решений однородного уравнения (1.2). Первые (но оставшиеся незамеченными) результаты о фредгольмовости оператора $\mathscr{L}$ с использованием свойства экспоненциальной дихотомии семейства эволюционных операторов были получены Ю.Я. Исаенко [24]. Им было доказано, что оператор $\mathscr{L}$ фредгольмов тогда и только тогда, когда семейство эволюционных операторов допускает экспоненциальную дихотомию на $\mathbb{R}_{+}$и $\mathbb{R}_{-}$. Этот же результат для оператора $\mathscr{L}=-d / d t+A(t)$ в банаховом пространстве $C_{b}\left(\mathbb{R}, \mathbb{C}^{n}\right)$ был получен в [25] K. Пальмером и в статье [26], в которой оператор $\mathscr{L}$ рассматривался в гильбертовом пространстве $L^{2}\left(\mathbb{R}, \mathbb{C}^{n}\right)$.

В следующих двух теоремах получены необходимые и достаточные условия фредгольмовости разностного оператора $\mathscr{D} \in \operatorname{End} \mathscr{F}(\mathbb{Z}, X)$, определенного формулой $(1.8)$, и оператора $\mathscr{L}=\mathscr{L}_{\mathscr{U}}: D(\mathscr{L}) \subset \mathscr{F}(\mathbb{R}, X) \rightarrow \mathscr{F}(\mathbb{R}, X)$.

Tеорема 1.15. Onератор $\mathscr{D} \in$ End $\mathscr{F}(\mathbb{Z}, X)$ фредголъмов тогда и только тогда, когда для семейства $\mathscr{U}=\mathscr{U}_{d}: \Delta_{\mathbb{Z}} \rightarrow$ End $X$ выполнено предположение 1.1 и узловой оператор $\mathscr{N}_{b, a}: \operatorname{Im} Q_{-}(a) \rightarrow \operatorname{Im} Q_{+}(b)$ фредгольмов. При условии фредгольмовости одного из них их индексы совпадают, а также совпадают размерности ядер и коразмерности образов.

Tеорема 1.16. Oператор $\mathscr{L}=\mathscr{L}_{\mathscr{U}} \oint$ федгольмов тогда и толъко тогда, когда для семейства эволюционных операторов $\mathscr{U}: \Delta_{\mathbb{R}} \rightarrow$ End $X$ выполнено предположение 1.1 и узловой оператор $\mathscr{N}_{b, a}: \operatorname{Im} Q_{-}(a) \rightarrow \operatorname{Im} Q_{+}(b)$ фредгольмов.

Достаточность условий теорем 1.15 и 1.16 была установлена в статьях [43], [21], она также следует из теорем 1.12, 1.13.

Доказательство необходимости условий теоремы вначале осуществлялось при дополнительных условиях (см. [27] для рефлексивного пространства $X$ и [17], где была приведена схема доказательства). В [28] было замечено, что эти дополнительные условия являются лишними.

В статье [29] необходимость условий теоремы 1.16 была доказана для пространств $L^{p}(\mathbb{R}, X), p \in[1, \infty)$, и $C_{0}(\mathbb{R}, X)$. Теорема 1.16 является новой для пространства $C_{b}(\mathbb{R}, X)$. Более подробно история вопроса, связанная с фредгольмовостью дифференциальных операторов, освещена в [19], [27]-[29], там же рассматривались примеры фредгольмовых операторов.

СлеДСтвие 1.2. Если оператор $\mathscr{D}$ (оператор $\mathscr{L})$ фредголъмов в одном из однородных пространств последовательностей (функиий), то он фредгольмов в любом однородном пространстве и его индекс (а также размерность ядра, коразмерность образа) не зависит от вида однородного пространства, где он действует.

Теорема 1.17. Если для семейства эволючионнъх операторов $\mathscr{U}: \Delta_{\mathbb{R}} \rightarrow$ End $X$ выполнено предположение 1.1 с $Q_{-}=0, Q_{+}=0$ (на $\mathbb{R}_{-, a} u \mathbb{R}_{b,+}$ имеет место тривиальная дихотомия), то оператор $\mathscr{L}$ непрерьвно обратим. 
Аналогичный результат верен и для оператора $\mathscr{D}$. Он получен в теореме 5.4. Теорема 1.17 будет следовать из теоремы 5.4 и теоремы 1.5.

СЛЕДСТВИЕ 1.3. Пусть для семейства эволюиионных операторов $\mathscr{U}: \Delta_{\mathbb{R}} \rightarrow$ End $X$ существуют в равномерной операторной топологии предель

$$
\lim _{t \rightarrow \pm \infty} \mathscr{U}(t, t-1)=\mathscr{B}_{ \pm} \in \text { End } X
$$

со спектральными радиусами $r\left(\mathscr{B}_{ \pm}\right)<1$. Тогда рассматриваемый оператор $\mathscr{L}: D(\mathscr{L}) \subset \mathscr{F}(\mathbb{R}, X) \rightarrow \mathscr{F}(\mathbb{R}, X)$ непрерьвно обратим в любом однородном пространстве $\mathscr{F}(\mathbb{R}, X)$.

СледствиЕ 1.4. Пусть семейство $\mathscr{U}: \Delta_{\mathbb{R}} \rightarrow$ End $X$ удовлетворяет условиям теоремы 1.17 и $B \in C_{0}(\mathbb{R}$, End $X)$. Тогда оператор $\mathscr{L}+\mathscr{B}$, где $\mathscr{B}$ оператор умножения в $\mathscr{F}(\mathbb{R}, X)$ на функиию $B$, непрерывно обратим.

Возможные приложения к уравнениям с частными производными достаточно подробно описаны в работах [8], [9], [19], [14]-[17], [27]-[29]. Приложения к исследованию уравнений Винера-Хопфа обсуждаются в [19], [28]. В разделе 8 рассматривается несколько примеров операторов, к которым применимы приведенные результаты.

\section{2. Однородные пространства функций}

2.1. Основные функциональные пространства. Рассмотрим несколько наиболее используемых нами пространств векторных функций, определенных на промежутке $\mathbb{J} \in\{[a, b],(-\infty, a],[a, \infty), \mathbb{R}\}$. Все они являются банаховыми и содержатся в линейном пространстве $L_{\text {loc }}^{1}(\mathbb{J}, X)$ локально суммируемых (измеримых по Бохнеру) на $\mathbb{J}$ функций со значениями в банаховом пространстве $X$. Приведем некоторые из них:

$L^{p}=L^{p}(\mathbb{J}, X), p \in[1, \infty],-\left(\right.$ банахово) пространство функций $x \in L_{\mathrm{loc}}^{1}(\mathbb{J}, X)$, для которых конечна величина (принимаемая за норму в соответствующем пространстве)

$$
\|x\|_{p}=\left(\int_{\mathbb{J}}\|x(\tau)\|^{p} d \tau\right)^{1 / p}, \quad p \neq \infty, \quad\|x\|_{\infty}=\operatorname{essip}_{\tau \in \mathbb{J}}\|x(\tau)\|, \quad p=\infty
$$

$S^{p}=S^{p}(\mathbb{J}, X), p \in[1, \infty),-$ пространство Степанова, состоящее из функций $x \in L_{\text {loc }}^{1}(\mathbb{J}, X)$, для которых конечна величина

$$
\|x\|_{S^{p}}=\sup _{t \in \mathbb{J}}\left(\int_{0}^{1}\|x(s+t)\|^{p} d s\right)^{1 / p}
$$

если $\mathbb{J} \in\left\{\mathbb{R}_{+}, \mathbb{R}\right\} ;$ для $\mathbb{J}=\mathbb{R}_{-a}$ промежуток интегрирования заменяется на отрезок $[a-1, a]$, а для $\mathbb{J}=\mathbb{R}_{a,+}-$ на отрезок $[a, a+1]$;

$C_{0}=C_{0}(\mathbb{J}, X)$ - подпространство функций $x$ из $C_{b}(\mathbb{J}, X)$, для которых выполнено свойство $\lim _{t \in \mathbb{J},|t| \rightarrow \infty}\|x(t)\|=0$.

Далее через $\mathbb{J}_{d}$ обозначается одно из множеств $\mathbb{Z}_{-}, \mathbb{Z}_{+}, \mathbb{Z}$, при этом $\mathbb{J}_{d}=\mathbb{Z}_{-}$, если $\mathbb{J}=\mathbb{R}_{-, a}, \mathbb{J}_{d}=\mathbb{Z}_{+}$, если $\mathbb{J}=\mathbb{Z}_{a,+}$, и $\mathbb{J}_{d}=\mathbb{Z}$, если $\mathbb{J}=\mathbb{R}$. 
Введем в рассмотрение еще одно семейство банаховых пространств (винеровская амальгама):

$$
L^{p, q}=L^{p, q}(\mathbb{J}, X), \quad p=0 \text { или } p \in[1, \infty], \quad q \in[1, \infty],
$$

где $\mathbb{J} \in\left\{\mathbb{R}_{-, a}, \mathbb{R}_{a,+}, \mathbb{R}\right\}$. Банахово пространство $L^{p, q}(\mathbb{R}, X)$ состоит из функций $x \in L_{\text {loc }}^{1}(\mathbb{R}, X)$, описываемых следующим образом. Функции $x$ поставим в соответствие последовательность $\left(x_{n}\right), n \in \mathbb{Z}_{+}$, где $x_{n} \in L^{1}([0,1], X)$, $x_{n}(s)=x(s+n), s \in[0,1]$. Функцию $x$ отнесем к пространству $L^{p, q}(\mathbb{R}, X)$, если $x_{n} \in L^{q}([0,1], X), n \in \mathbb{Z}$, а сама последовательность $\left(x_{n}\right)$ принадлежит $\ell^{p}\left(\mathbb{Z}, L^{q}([0,1], X)\right)$. При $p=0$ последовательность $\left(x_{n}\right)$ считается принадлежащей пространству $c_{0}\left(\mathbb{Z}, L^{q}([0,1], X)\right)$. Нормой функции $x \in L^{p, q}(\mathbb{R}, X)$ полагаем

$$
\|x\|_{p, q}= \begin{cases}\left\|\left(x_{n}\right)\right\|_{p}=\left(\sum_{n=\infty}^{\infty}\left\|x_{n}\right\|_{q}^{p}\right)^{1 / p}, & \text { если } p \in[1, \infty), q \in[1, \infty], \\ \sup _{n \in \mathbb{Z}}\left\|\left(x_{n}\right)\right\|_{q}, & \text { если } p \in\{0, \infty\}, q \in[1, \infty] .\end{cases}
$$

Аналогично определяются пространства $L^{p, q}(\mathbb{J}, X)$ для $\mathbb{J} \in\left\{\mathbb{R}_{-, a}, \mathbb{R}_{a,+}\right\}$. Для $x \in L^{p, q}(\mathbb{J}, X)$ последовательность $\left(x_{n}\right)$ будет односторонней. Ясно, что $L^{p, p}(\mathbb{J}, X)=L^{p}(\mathbb{J}, X), p \in[1, \infty]$, и банахово пространство $L^{\infty, q}(\mathbb{J}, X)$ совпадает с пространством Степанова $S^{q}(\mathbb{J}, X), q \in[1, \infty)$. Если $X \in\{\mathbb{R}, \mathbb{C}\}$, то в обозначениях пространств убирается символ $X$. Например, $L^{p, q}(\mathbb{J})=L^{p}(\mathbb{J}, X)$.

2.2. Однородные пространства функций и последовательностей. Далее аксиоматизируется класс функциональных пространств, в которых действуют линейные операторы, построенные по семейству эволюционных операторов $\mathscr{U}: \Delta_{\mathbb{J}} \rightarrow$ End $X$, где $\mathbb{J} \in\left\{\mathbb{R}_{-, a}, \mathbb{R}_{a,+}, \mathbb{J}\right\}, a \in \mathbb{R}$. Через $\mathbb{J}(n)$ обозначается промежуток вида

$$
\mathbb{J}(n)= \begin{cases}{[n, n+1), n \in \mathbb{Z},} & \text { если } \mathbb{J}=\mathbb{R}, \\ {[a+n, a+n+1), n \in \mathbb{Z}_{+},} & \text {если } \mathbb{J}=\mathbb{R}_{a,+}, \\ (a+n-1, a+n], n \in \mathbb{Z}_{-}, & \text {если } \mathbb{J}=\mathbb{R}_{-, a} .\end{cases}
$$

ОПредЕЛЕНиЕ 2.1. Банахово пространство $\mathscr{F}(\mathbb{J})$ измеримых на $\mathbb{\Xi}$ вещественных функций назовем однородным пространством измеримых функций, если выполнены следующие условия:

1) $\mathscr{F}(\mathbb{J})$ содержится в $L_{\mathrm{loc}}^{1}(\mathbb{J})=L_{\mathrm{loc}}^{1}(\mathbb{J}, \mathbb{R})$, причем $\int_{b}^{b+1}|x(\tau)| d \tau \leqslant c\|x\|_{\mathscr{F}(\mathbb{R})}$, $x \in \mathscr{F}(\mathbb{R})$, где $c>0$, если $[b, b+1] \subset \mathbb{J} ;$

2) $\mathscr{F}(\mathbb{J})$ - банахова решетка; это означает, что если $\psi \in \mathscr{F}(\mathbb{J})$ и $\varphi: \mathbb{J} \rightarrow \mathbb{R}-$ измеримая функция такая, что $|\varphi(t)| \leqslant|\psi(t)|$ для почти всех $t \in \mathbb{R}_{+}$, то $\varphi \in$ $\mathscr{F}(\mathbb{J})$, причем $\|\varphi\| \leqslant\|\psi\| ;$

3) для $\mathbb{J}=\mathbb{R}$ определены и ограничены операторы сдвига функций $S(t) \in$ End $\mathscr{F}(\mathbb{J})$ вида

$$
(S(t) x)(\tau)=x(\tau+t), \quad \tau, t \in \mathbb{J}, \quad x \in \mathscr{F}(\mathbb{J}) ;
$$


для $\mathbb{J}=\mathbb{R}_{a,+}$ определены и ограничены операторы вида $(2.1)$, а для $t<0$ из $\mathbb{J}$ - также операторы

$$
\left(S_{+}(t) x\right)(\tau)= \begin{cases}x(\tau+t), & \text { если } \tau+t \geqslant a, \\ 0, & \text { если } a<\tau+t\end{cases}
$$

для $\mathbb{J}=\mathbb{R}_{-, a}$ и $t \leqslant 0$ определены и ограничены операторы $(2.1)$, а для $t>0$ из $\mathbb{J}$ - операторы

$$
\left(S_{-}(t) x\right)(\tau)= \begin{cases}0, & \text { если } \tau+t>a, \\ x(\tau+t), & \text { если } \tau+t \geqslant a ;\end{cases}
$$

при этом $\sup _{t \in \mathbb{R}}\|S(t)\|<\infty, \sup _{t 0}\left\|S_{-}(t)\right\|<\infty$;

4) характеристическая функция $\chi_{[b, b+1]}$ отрезка $[b, b+1](b=0$, если $\mathbb{J}=\mathbb{R}$; $b=a$, если $\mathbb{J}=\mathbb{R}_{a,+} ; b=-a$, если $\left.\mathbb{J}=\mathbb{R}_{-, a}\right)$ принадлежит пространству $\mathscr{F}(\mathbb{J}, X)$, и для любой функции $x \in \mathscr{F}(\mathbb{J})$ функция $\widetilde{x}: \mathbb{R} \rightarrow \mathbb{R}$, определенная равенствами

$$
\widetilde{x}(t)=\left\|\chi_{\mathbb{J}(n)} x\right\|_{S^{1}(\mathbb{J})}, \quad t \in \mathbb{J}(n), \quad n \in \mathbb{J}_{d},
$$

принадлежит пространству $\mathscr{F}(\mathbb{J})$ и $\|\widetilde{x}\| \leqslant\|x\|$.

Рассмотрим линейное подпространство $\mathscr{F}_{s}(\mathbb{J})$ функций из однородного пространства $\mathscr{F}(\mathbb{J})$, принимающих постоянное значение на каждом интервале $\mathbb{J}(n)$, $n \in \mathbb{J}_{d}$. По этому замкнутому подпространству $\mathscr{F}_{s}(\mathbb{J})$ определим банахово пространство $\mathscr{F}_{s}\left(\mathbb{J}_{d}\right)$ вещественных последовательностей $x: \mathbb{J}_{d} \rightarrow \mathbb{R}$ таких, что функция $\widetilde{x}: \mathbb{J} \rightarrow \mathbb{R}$, определенная равенством $\widetilde{x}(t)=x(n)$, если $t \in \mathbb{J}(n), n \in \mathbb{J}_{d}$, принадлежит пространству $\mathscr{F}_{s}(\mathbb{J})$, и положим $\|x\|=\|\widetilde{x}\|_{\mathscr{F}(\mathbb{J})}$. Получившееся банахово пространство последовательностей $\mathscr{F}\left(\mathbb{J}_{d}\right)$ назовем пространством, ассоииированным с $\mathscr{F}(\mathbb{J})$. Оно в силу своего определения обладает свойствами, аналогичными свойствам однородного пространства $\mathscr{F}(\mathbb{J})$.

ОПРЕДЕЛЕНИЕ 2.2. Банахово пространство $\mathscr{F}(\mathbb{J}, X)$ измеримых (по Бохнеру) функций $x: \mathbb{J} \rightarrow X$, для которых функция $\bar{x}: \mathbb{J} \rightarrow \mathbb{R}, \bar{x}(t)=\|x(t)\|$, $t \in \mathbb{J}$, принадлежит $\mathscr{F}(\mathbb{J})$ и $\|x\|=\|\bar{x}\|_{\mathscr{F}(\mathbb{J})}$, назовем однородным пространством измеримых векторных функиий, соответствующих однородному пространству $\mathscr{F}(\mathbb{J})$.

ЗАмЕчАниЕ 2.1. Если $\mathscr{F}(\mathbb{J}, X)$ - однородное пространство функций, отвечающих однородному пространству $\mathscr{F}(\mathbb{J})$, то оно обладает соответствующими свойствами 1)-4) из определения 2.1. Через $\mathscr{F}\left(\mathbb{J}_{d}, X\right)$ обозначим банахово пространство последовательностей $x: \mathbb{J}_{d} \rightarrow X$, обладающих следующими свойствами: последовательность $\widetilde{x}(n)=\|x(n)\|, n \in \mathbb{J}_{d}$, принадлежит пространству $\mathscr{F}\left(\mathbb{J}_{d}\right)$ и $\|x\|_{\mathscr{F}\left(\mathbb{J}_{d}, X\right)}=\|\bar{x}\|_{\mathscr{F}\left(\mathbb{J}_{d}\right)}$. Банахово пространство $\mathscr{F}\left(\mathbb{J}_{d}, X\right)$ можно определить как совокупность последовательностей функций $x: \mathbb{J}_{d} \rightarrow X$ таких, что функция $y: \mathbb{J} \rightarrow X, y(t)=x(n), t \in \mathbb{J}(n), n \in \mathbb{J}_{d}$, принадлежит $\mathscr{F}(\mathbb{J}, X)$. Положим $\|x\|_{\mathscr{F}\left(\mathbb{J}_{d}, X\right)}=\|y\|_{\mathscr{F}(\mathbb{J})}$. Получившееся пространство последовательностей будем называть однородным пространством последовательностей, ассоциированным с $\mathscr{F}(\mathbb{J}, X)$. Пара пространств $\mathscr{F}(\mathbb{J}, X), \mathscr{F}\left(\mathbb{J}_{d}, X\right)$ называется ассоииированной парой. 
ЗАмечАниЕ 2.2. Банаховы пространства

$$
S^{p}(\mathbb{J}, X), \quad p \in[1, \infty) ; \quad L^{p, q}(\mathbb{J}, X), \quad p=0 \text { или } p \in[1, \infty], \quad q \in[1, \infty],
$$

являются однородными пространствами измеримых функций, а ассоциированными с ними пространствами последовательностей являются соответственно пространства $\ell^{\infty}\left(\mathbb{J}_{d}, X\right)$ и $\ell^{p}\left(\mathbb{J}_{d}, X\right)$, если $p \neq 0$. Если $p=0$, то пространству $L^{0, q}(\mathbb{J}, X)$ соответствует пространство $c_{0}\left(\mathbb{J}_{d}, X\right)$.

ЗАмечАниЕ 2.3. Непосредственно из определения 2.2 следует, что верны включения

$$
L^{1, \infty}(\mathbb{J}, X) \subset \mathscr{F}(\mathbb{J}, X) \subset S^{1}(\mathbb{J}, X)
$$

для любого однородного пространства $\mathscr{F}(\mathbb{J}, X)$ и включения

$$
\ell^{1}\left(\mathbb{J}_{d}, X\right) \subset \mathscr{F}\left(\mathbb{J}_{d}, X\right) \subset \ell^{\infty}\left(\mathbb{J}_{d}, X\right)
$$

для однородного пространства последовательностей $\mathscr{F}\left(\mathbb{J}_{d}, X\right)$ (ассоциированного с $\mathscr{F}(\mathbb{J}, X))$.

ЗАмЕчАниЕ 2.4. Если $\mathscr{F}(\mathbb{R}, X)$ - однородное пространство функций, то для любых $\alpha \neq 0$ и $\beta$ из $\mathbb{R}$ пространство функций $\mathscr{F}_{\alpha, \beta}(\mathbb{R}, X)=\{y(t)=x(\alpha t+\beta)$, $t \in \mathbb{R} \mid x \in \mathscr{F}(\mathbb{R}, X)\}$ с нормой $\|y\|=\|x\|$ также является однородным.

Кроме однородных пространств измеримых функций в статье рассматривается банахово пространство ограниченных непрерывных функций $C_{b}(\mathbb{J}, X)$ и его подпространство $C_{0}(\mathbb{J}, X)=\left\{x \in C_{b}(\mathbb{J}, \mathbb{X}) \mid \lim _{|t| \rightarrow \infty}\|x(t)\|=0\right\}$.

Символом $\mathscr{F}(\mathbb{J}, X)$ мы будем обозначать как однородное пространство измеримых функций, так и $C_{b}(\mathbb{J}, X)$, а также $C_{0}(\mathbb{J}, X)$. Ассоциированными с пространствами $C_{b}(\mathbb{J}, X), C_{0}(\mathbb{J}, X)$ будут являться пространства последовательностей $\ell^{\infty}\left(\mathbb{J}_{d}, X\right)$ и $c_{0}\left(\mathbb{J}_{d}, X\right)$ соответственно. В [1] были введены понятия однородного пространства измеримых функций $\mathscr{F}\left(\mathbb{R}_{+}, X\right)$ и однородного пространства векторных последовательностей. В [1] были выделены подпространства, важные для исследования рассматриваемых операторов и отношений, а также получены оценки норм операторов, действующих в однородных пространствах функций и последовательностей. Все эти понятия и результаты естественным образом по аналогии вводятся и получаются и для однородных пространств функций и последовательностей, определенных на $\mathbb{J} \in\left\{\mathbb{R}_{-, a}, \mathbb{R}\right\}, \mathbb{J}_{d} \in\left\{\mathbb{Z}_{-}, \mathbb{Z}\right\}$ соответственно. При доказательствах утверждений используются приводимые в [1] оценки норм.

Пусть $\mathscr{F}(\mathbb{J}, X)$ - однородное пространство измеримых функций или же одно из пространств $C_{b}(\mathbb{J}, X), C_{0}(\mathbb{J}, X)$. Символом $\mathscr{F}_{c}(\mathbb{J}, X)$ обозначим наименьшее замкнутое подпространство из $\mathscr{F}(\mathbb{J}, X)$, содержащее функции из $\mathscr{F}(\mathbb{J}, X)$ с компактным носителем. Если $\mathscr{F}\left(\mathbb{J}_{d}, X\right)$ - однородное пространство последовательностей, то через $\mathscr{F}_{c}\left(\mathbb{J}_{d}, X\right)$ обозначается наименьшее подпространство из $\mathscr{F}\left(\mathbb{J}_{d}, X\right)$, содержащее финитные последовательности из $\mathscr{F}\left(\mathbb{J}_{d}, X\right)$. Если $\mathscr{F}(\mathbb{J}, X)=C_{b}(\mathbb{J}, X)$, то положим $\mathscr{F}_{c}(\mathbb{J}, X)=C_{0}(\mathbb{J}, X)$.

Однородные пространства $\mathscr{F}=L^{p, q}(\mathbb{J}, X), p=0, p \in[1, \infty), q \in[1, \infty]$, и $\mathscr{F}=$ $L^{p}(\mathbb{J}, X), p \in[1, \infty)$, таковы, что для них $\mathscr{F}=\mathscr{F}_{c}$. Пространство $\left(S^{p}(\mathbb{J}, X)\right)_{c}$, $p \in[1, \infty)$, совпадает с $L^{0, p}(\mathbb{J}, X)$, а $\left(L^{\infty}(\mathbb{J}, X)\right)_{c}=L^{0, \infty}(\mathbb{J}, X)$. Кроме того, отметим, что $\left(\ell^{p}\left(\mathbb{J}_{d}, X\right)\right)_{c}=\left(\ell^{p}\left(\mathbb{J}_{d}, X\right)\right), p \in[1, \infty)$, и $\left(\ell^{\infty}\left(\mathbb{J}_{d}, X\right)\right)_{c}=c_{0}\left(\mathbb{J}_{d}, X\right)$. 


\section{3. О состояниях оператора $\mathscr{N}_{a}^{+}$и отношения $\mathscr{D}_{E}^{+}$; доказательство теорем 1.7-1.11}

Большинство используемых понятий и результатов теории линейных отношений содержатся в статьях [19], [30] (см. также монографии [31], [32]). Для линейного отношения $\mathscr{A} \in L R(\mathscr{X}, \mathscr{Y})$ его сопряженным является отношение $\mathscr{A}^{*} \in L R\left(\mathscr{Y}^{*}, \mathscr{X}^{*}\right)$, определенное формулой

$$
\mathscr{A}^{*}=\left\{(\eta, \xi) \in \mathscr{Y}^{*} \times \mathscr{X}^{*} \mid\langle y, \eta\rangle=\langle x, \xi\rangle \forall(x, y) \in \mathscr{A}\right\} .
$$

Используемые свойства сопряженного отношения имеются в работах [19], [30]-[38].

3.1. О ядрах и образах оператора $\mathscr{N}_{a}^{+}$и отношения $\mathscr{D}_{E}^{+}$. В первой части раздела 3 рассматривается дискретное семейство эволюционных операторов $\mathscr{U}=\mathscr{U}_{d}: \Delta_{\mathbb{Z}_{+}} \rightarrow$ End $X$, для которого выполнено следующее предположение.

ПреДПОЛОЖеНИЕ 3.1. Существует число $а \in \mathbb{Z}_{+}$такое, что семейство $\mathscr{U}$ допускает экспоненциальную дихотомию на множестве $\mathbb{Z}_{a,+}$ с расщепляющей парой проекторнозначных функций $P_{+}, Q_{+}: \mathbb{Z}_{a,+} \rightarrow$ End $X$.

Без ограничения общности будем далее считать, что $a=0$, т. е. далее будет рассматриваться отношение $\mathscr{D}_{E}^{+}$. Его ядро допускает представление

$$
\operatorname{Ker} \mathscr{D}_{E}^{+}=\left\{x \in \mathscr{F}\left(\mathbb{Z}_{+}, X\right) \mid x(n)=\mathscr{U}(n, 0) x_{0}, n \geqslant 0, x_{0} \in E\right\} .
$$

Введем в рассмотрение ограниченный оператор $B_{d}: X \rightarrow \mathscr{F}\left(\mathbb{Z}_{+}, X\right)$ вида

$$
\left(B_{d} x\right)(n)=\left\{\begin{array}{ll}
\mathscr{U}(n, 0) x, & 0 \leqslant n \leqslant a-1, \\
\mathscr{U}(n, 0) P_{+}(a) x, & n \geqslant a,
\end{array} \quad n \in \mathbb{Z}_{+}, \quad x \in X .\right.
$$

ЗАмечАниЕ 3.1. Непосредственно из вида оператора $B_{d}$ следует, что последовательность $B_{d} x$ принадлежит замкнутому подпространству Ker $\mathscr{D}_{E}^{+}$из $\mathscr{F}\left(\mathbb{Z}_{+}, X\right)$ тогда и только тогда, когда вектор $x \in X$ принадлежит подпространству $\operatorname{Ker} \mathscr{N}_{a}^{+}$из $X$, где оператор $\mathscr{N}_{a}^{+}$определен формулой (1.17).

Из замечания 3.1 вытекает следующее утверждение.

Лемма 3.1. Оператор $B_{d}: X \rightarrow \mathscr{F}\left(\mathbb{Z}_{+}, X\right)$ осуществляет изоморфизм подпространств $\operatorname{Ker} \mathscr{N}_{a}^{+} u \operatorname{Ker} \mathscr{D}_{E}^{+}$.

Из леммы 3.1 и замечания 3.1 вытекает следующее утверждение.

Лемма 3.2. Если подпространство $\operatorname{Ker} \mathscr{D}_{E}^{+}$из $\mathscr{F}\left(\mathbb{Z}_{+}, X\right)$ является дополняемым в $\mathscr{F}\left(\mathbb{Z}_{+}, X\right)$ и $\mathscr{F}_{0}$ - его замкнутое дополнение, т.е.

$$
\mathscr{F}\left(\mathbb{Z}_{+}, X\right)=\operatorname{Ker} \mathscr{D}_{E}^{+} \oplus \mathscr{F}_{0},
$$

то подпространство $\operatorname{Ker} \mathscr{N}_{a}^{+}$из $X$ дополняемо в $X$ и имеет место равенство

$$
X=\operatorname{Ker} \mathscr{N}_{a}^{+} \oplus B_{d}^{-1}\left(\mathscr{F}_{0}\right) .
$$


При описании подпространства $\operatorname{Im} \mathscr{D}_{E}^{+}$будут использоваться линейные ограниченные операторы $\Phi_{a}^{0}, \Phi_{a}: \mathscr{F}\left(\mathbb{Z}_{+}, X\right) \rightarrow X$, определенные формулами

$$
\begin{gathered}
\Phi_{a}^{0} x=Q_{+}(a) \sum_{k=0}^{a-1} \mathscr{U}(a, k) x(k)+\sum_{k \geqslant a+1} \mathscr{U}(a, k) Q_{+}(k) x(k), \\
\Phi_{a} x=\Phi_{a}^{0} x+Q_{+}(a) x(a), \quad x \in \mathscr{F}\left(\mathbb{Z}_{+}, X\right) .
\end{gathered}
$$

Лемма 3.3. Для образа $\operatorname{Im} \mathscr{D}_{E}^{+}$отношения $\mathscr{D}_{E}^{+}$справедливы равенства

$$
\begin{aligned}
\operatorname{Im} \mathscr{D}_{E}^{+}= & \left\{z \in \mathscr{F}\left(\mathbb{Z}_{+}, X\right) \mid z(n)=y(n), n \neq a, u z(a)=y_{a}-\Phi_{a}^{0} y+\mathscr{N}_{a}^{+} x_{0}\right. \\
& \text { } \left.\text { лля некоторьх y } \in \mathscr{F}\left(\mathbb{Z}_{+}, X\right), y_{a} \in \operatorname{Im} P_{+}(a) u x_{0} \in E\right\}, \\
\operatorname{Im} \mathscr{D}_{E}^{+}= & \Phi_{a}^{-1}\left(\operatorname{Im} \mathscr{N}_{a}^{+}\right) .
\end{aligned}
$$

3.2. Доказательство теорем 1.7 и 1.8. Совпадение состояний отношений $\mathscr{D}_{E}^{+}$и оператора $\mathscr{N}_{a}^{+}$(равенства (1.16)) следует из лемм 3.1-3.3. Из леммы 3.1 следует совпадение размерностей ядер отношения $\mathscr{D}_{E}^{+}$и оператора $\mathscr{N}_{a}^{+}$. Из леммы 3.3 следует совпадение коразмерностей подпространств $\operatorname{Im} \mathscr{D}_{E}^{+}$ и $\operatorname{Im} \mathscr{N}_{a}^{+}$соответственно в $\mathscr{F}\left(\mathbb{Z}_{+}, X\right)$ и $\operatorname{Im} Q_{+}(a)$.

Для доказательства теоремы 1.8 достаточно сослаться на доказанную теорему 1.7 и теорему 1.3, которая (независимо) доказывается в разделе 4.

3.3. Доказательство теорем 1.9-1.11. Из теоремы 5.7 статьи [19] следует, что оператор $\mathscr{L}_{E}^{+}$и отношение $\mathscr{D}_{E}^{+}$одновременно фредгольмовы и их индексы совпадают. Нетрудно видеть, что фредгольмовость отношения $\mathscr{D}_{E}^{+}$влечет экспоненциальную дихотомию семейства $\mathscr{U}$ на $\mathbb{Z}_{a,+}$ при некотором $a \in \mathbb{Z}_{+}$. Из [19; лемма 6.2] получаем, что $\mathscr{U}$ допускает экспоненциальную дихотомию на $\mathbb{R}_{a,+}$. Равенства (1.19) следуют из равенства (1.18) теоремы 1.8. Теорема 1.9 доказана.

Теоремы 1.10 и 1.11 непосредственно следуют из теоремы 1.9.

\section{4. Доказательство теорем 1.2, $1.3,1.5$}

Рассматриваются ассоциированная пара $\mathscr{F}(\mathbb{R}, X), \mathscr{F}(\mathbb{Z}, X)$ однородных пространств и операторы $\mathscr{L}: D(\mathscr{L}) \subset \mathscr{F}(\mathbb{R}, X) \rightarrow \mathscr{F}(\mathbb{R}, X), \mathscr{D} \in \operatorname{End} \mathscr{F}(\mathbb{Z}, X)$, определенные в разделе 1 (разностный оператор $D$ определялся формулой (1.8)) по семейству эволюционных операторов $\mathscr{U}: \Delta_{\mathbb{R}} \rightarrow$ End $X$. Устанавливается ряд лемм и теорем об их ядрах и образах, из которых будет следовать теорема 1.5. Соответствующие леммы и теоремы для оператора $\mathscr{L}_{E}^{+}$и отношения $\mathscr{D}_{E}^{+}$были установлены в [19; §5]. Поскольку здесь мы придерживаемся той же схемы доказательств и часть утверждений для операторов $\mathscr{L}$ и $\mathscr{D}$ (для широкого класса однородных пространств) была фактически установлена в статье [14], то доказательство некоторых утверждений будет либо существенно сокращено, либо опущено. При этом делаются ссылки на соответствующие утверждения из [14] и [19]. 
4.1. Доказательство теорем 1.2, 1.5. Доказательство теоремы 1.2 проводится аналогично доказательству теоремы 2 из статьи [14]. Ограниченность рассматриваемых в $\mathscr{F}(\mathbb{R}, X)$ операторов фактически установлена в [19; 2$]$.

Доказательство теоремы 1.5 использует оператор $B: \mathscr{F}(\mathbb{Z}, X) \rightarrow \mathscr{F}(\mathbb{R}, X)$, $(B x)(s)=-\varphi(s) \mathscr{U}(s, n-1) x(n-1), n \in \mathbb{Z}, s \in[n-1, n]$ (где $\varphi: \mathbb{R} \rightarrow \mathbb{R}-$ периодическая периода 1 функция, для которой $\varphi(s)=6 s(1-s), s \in[0,1])$, осуществляющего изоморфизм ядер $\operatorname{Ker} \mathscr{L}$ и $\operatorname{Ker} \mathscr{D}$ операторов $\mathscr{L}$ и $\mathscr{D}$. Для описания образов операторов $\mathscr{L}$ и $\mathscr{D}$ применяются ограниченные операторы

$$
\begin{gathered}
\mathscr{B}: \mathscr{F}(\mathbb{Z}, X) \rightarrow \mathscr{F}(\mathbb{R}, X), \quad \mathscr{B} x=-\varphi B x, \quad x \in \mathscr{F}(\mathbb{Z}, X), \\
C: \mathscr{F}(\mathbb{R}, X) \rightarrow \mathscr{F}(\mathbb{Z}, X), \\
(C y)(n)=-\int_{n-1}^{n} \mathscr{U}(n, \tau) y(\tau) d \tau, \quad n \in \mathbb{Z}, \quad y \in \mathscr{F}(\mathbb{R}, X) .
\end{gathered}
$$

Применение этих операторов к описанию образов $\operatorname{Im} \mathscr{L}, \operatorname{Im} \mathscr{D}$ операторов осуществляется по той же схеме, что и в леммах 5.7-5.12 и теоремах 5.1-5.6 из статьи [1], где рассматривался оператор $\mathscr{L}_{E}^{+}$и отношение $\mathscr{D}_{E}^{+}$. Непосредственно из аналогов этих результатов следует утверждение теоремы 1.5 , т. е. верны равенства (1.14), (1.15).

\section{2. Доказательство теоремы 1.3.}

ЗАмечАниЕ 4.1. Рассматриваемый в теореме 1.3 оператор $\mathscr{L}_{E}^{+}: D\left(\mathscr{L}_{E}^{+}\right) \subset$ $\mathscr{F}\left(\mathbb{R}_{+}, X\right) \rightarrow \mathscr{F}\left(\mathbb{R}_{+}, X\right)$ может быть незамкнутым (в [19] приведен соответствующий пример), и он замкнут тогда и только тогда, когда замкнуто его ядро $\operatorname{Ker} \mathscr{L}_{E}^{+}$. В связи с этим фактом возникает проблема интерпретации в формулировке теоремы 1.3 свойства 3 ) из определения 1.1. В теореме 1.3 это свойство по отношению к оператору $\mathscr{L}_{E}^{+}$понимается так: $\operatorname{Ker} \mathscr{L}_{E}^{+}-$дополняемое подпространство либо в $\mathscr{F}\left(\mathbb{R}_{+}, X\right)$, либо в $D\left(\mathscr{L}_{E}^{+}\right)$с нормой (см. [19])

$$
\|x\|=\|x(0)\|_{X}+\|x\|_{\mathscr{F}}+\left\|\mathscr{L}_{E}^{+} x\right\|_{\mathscr{F}}, \quad x \in D\left(\mathscr{L}_{E}^{+}\right) .
$$

Пространство $D\left(\mathscr{L}_{E}^{+}\right)$с такой нормой является банаховым. Если $\mathscr{F}\left(\mathbb{R}_{+}, X\right)=$ $C_{b}\left(\mathbb{R}_{+}, X\right)$, то оператор $\mathscr{L}_{E}^{+}$замкнут и норма $(4.1)$ в $D\left(\mathscr{L}_{E}\right)$ эквивалентна норме графика в $D\left(\mathscr{L}_{E}^{+}\right)$. В последующих утверждениях рассматривается пространство $D\left(\mathscr{L}_{E}^{+}\right)$с нормой (4.1). Подпространство Ker $\mathscr{L}_{E}^{+}$является замкнутым в $D\left(\mathscr{L}_{E}^{+}\right)$с нормой $(4.1)$.

При доказательстве следующего утверждения используется представление функций из $D\left(\mathscr{L}_{E}^{+}\right)$, полученное в $\left[19 ;\right.$ формула (5.4)]. Пусть $\lambda_{0} \in \mathbb{C}$ выбрано так, что $\operatorname{Re} \lambda_{0}>\alpha$, где число $\alpha \in \mathbb{R}$ взято из определения семейства эволюционных операторов (см. раздел 1). Тогда оператор $\mathscr{L}_{\{0\}}^{+}-\lambda_{0} I$ непрерывно обратим и каждая функция $x \in D\left(\mathscr{L}_{E}^{+}\right)$представима в виде

$$
\begin{aligned}
x(t) & =\left(\left(\mathscr{L}_{\{0\}}^{+}-\lambda_{0} I\right)^{-1}\left(\mathscr{L}_{E}^{+}-\lambda_{0} I\right) x\right)(t)+e^{-\lambda_{0} t} \mathscr{U}(t, 0) x(0) \\
& =-\int_{0}^{t} e^{-\lambda_{0}(t-\tau)} \mathscr{U}(t, \tau)\left(\left(\mathscr{L}_{E}^{+}-\lambda_{0} I\right) x\right)(\tau) d \tau+e^{-\lambda_{0} t} \mathscr{U}(t, 0) x(0), \quad t \geqslant 0,
\end{aligned}
$$


где $x(0) \in E$. Из представления (4.2) следует корректность определения и ограниченность оператора

$$
J_{+}: D\left(\mathscr{L}_{E}^{+}\right) \rightarrow \mathscr{F}\left(\mathbb{Z}_{+}, X\right), \quad\left(J_{+} x\right)(n)=x(n), \quad n \in \mathbb{Z}_{+}, \quad x \in D\left(\mathscr{L}_{E}^{+}\right) .
$$

Теорема 4.1. Если подпространство $\operatorname{Ker} \mathscr{D}_{E}^{+}$дополняемо в $\mathscr{F}\left(\mathbb{Z}_{+}, X\right)$ u

$$
\mathscr{F}\left(\mathbb{Z}_{+}, X\right)=\operatorname{Ker} \mathscr{D}_{E}^{+} \oplus \mathscr{F}_{0},
$$

где $\mathscr{F}_{0}$ - замкнутое подпространство из $\mathscr{F}\left(\mathbb{Z}_{+}, X\right)$, то Ker $\mathscr{L}_{E}^{+}-$дополняемое подпространство в $\mathscr{D}\left(\mathscr{L}_{E}^{+}\right)$(с нормой $\left.(4.1)\right)$ и

$$
D\left(\mathscr{L}_{E}^{+}\right)=\operatorname{Ker} \mathscr{L}_{E}^{+} \oplus J_{+}^{-1}\left(\mathscr{F}_{0}\right)
$$

ДоказАтельство. Из определения оператора $J_{+}$следует, что он осуществляет изоморфизм подпространств $\operatorname{Ker} \mathscr{L}_{E}^{+}, \operatorname{Ker} \mathscr{D}_{E}^{+}$. Если $y \in \operatorname{Ker} \mathscr{L}_{E}^{+} \cap J_{+}^{-1}\left(\mathscr{F}_{0}\right)$, то $J_{+} y \in \mathscr{F}_{0}$ и $J_{+} y \in \operatorname{Ker} \mathscr{D}_{E}^{+}$. Поэтому $y=0$. Из отмеченного следует, что разложение (4.4) вытекает из разложения (4.3). Теорема доказана.

В следующей теореме используется линейный оператор $\Phi_{E}: \mathscr{D}_{E}^{+} \rightarrow D\left(\mathscr{L}_{E}^{+}\right)$, построенный в [19; §5], для которого в лемме 5.9 из [19] было установлено равенство

$$
\mathscr{L}_{E}^{+} \Phi_{E}\left(x_{0}, f\right)=\mathscr{B}^{+} f, \quad\left(x_{0}, f\right) \in \mathscr{D}_{E}^{+},
$$

где оператор $\mathscr{B}^{+}: \mathscr{F}\left(\mathbb{Z}_{+}, X\right) \rightarrow \mathscr{F}\left(\mathbb{R}_{+}, X\right)$ был определен таким же образом, как и оператор $\mathscr{B}: \mathscr{F}(\mathbb{Z}, X) \rightarrow \mathscr{F}(\mathbb{R}, X)$.

Теорема 4.2. Пусть $\operatorname{Ker} \mathscr{L}_{E}^{+}$- замкнутое дополняемое в $D\left(\mathscr{L}_{E}^{+}\right)$с нормой (4.1) подпространство и

$$
D\left(\mathscr{L}_{E}^{+}\right)=\operatorname{Ker} \mathscr{L}_{E}^{+} \oplus \widetilde{\mathscr{F}},
$$

где $\widetilde{\mathscr{F}}$ - замкнутое подпространство из $D\left(\mathscr{D}_{E}^{0}\right)$. Тогда банахово пространство $\mathscr{F}\left(\mathbb{Z}_{+}, X\right)$ представимо в виде прямой суммы

$$
\mathscr{F}\left(\mathbb{Z}_{+}, X\right)=\operatorname{Ker} \mathscr{D}_{E}^{+} \oplus \widetilde{\mathscr{F}_{d}},
$$

где

$$
\begin{gathered}
\widetilde{\mathscr{F}}_{d}=\mathscr{P}_{1} \mathscr{D}_{E}^{0}, \quad \mathscr{D}_{E}^{0}=\Phi_{E}^{-1}(\widetilde{\mathscr{F}}), \\
\mathscr{P}_{1}: \mathscr{F}\left(\mathbb{Z}_{+}, X\right) \times \mathscr{F}\left(\mathbb{Z}_{+}, X\right) \rightarrow \mathscr{F}\left(\mathbb{Z}_{+}, X\right), \quad \mathscr{P}_{1}\left(x_{1}, x_{2}\right)=x_{1}, \\
\left(x_{1}, x_{2}\right) \in \mathscr{F}\left(\mathbb{Z}_{+}, X\right) \times \mathscr{F}\left(\mathbb{Z}_{+}, X\right) .
\end{gathered}
$$

ДокАЗАтЕльство. Из равенства (4.5) следует непрерывность оператора $\Phi_{E}: \mathscr{D}_{E}^{+} \rightarrow D\left(\mathscr{L}_{E}^{+}\right)$. Поскольку подпространство $\mathscr{D}_{E}^{0}$ замкнуто в $\mathscr{F}\left(\mathbb{Z}_{+}, X\right) \times$ $\mathscr{F}\left(\mathbb{Z}_{+}, X\right)$, то из определения оператора $\mathscr{P}_{1}$ следует замкнутость подпространства $\widetilde{\mathscr{F}} d$. Теперь для доказательства равенства (4.6) достаточно заметить, что областью определения отношения $\mathscr{D}_{E}^{+}$является все пространство $\mathscr{F}\left(\mathbb{Z}_{+}, X\right)$ и для любой пары $x_{0}, f \in \mathscr{F}\left(\mathbb{Z}_{+}, X\right)$ выполнено свойство: $\Phi_{E}\left(x_{0}, f\right) \in \operatorname{Ker} \mathscr{L}_{E}^{+}$ тогда и только тогда, когда $x_{0} \in \operatorname{Ker} \mathscr{D}_{E}^{+}$. Оно следует из способа построения оператора $\Phi_{E}$. Теорема доказана. 
ДОКАЗАТЕЛЬСтво тЕОРЕмЫ 1.3. ОДновременное совпадение свойств из определения 1.1 оператора $\mathscr{L}_{E}^{+}$и отношения $\mathscr{D}_{E}^{+}$и, в частности, равенства $(1.12)$, (1.13) следуют из результатов статьи $[19 ; \S 5]$ и доказанных здесь теорем 4.1 и 4.2. Теорема доказана.

\section{5. О состояниях оператора $\mathscr{D}$ и узлового оператора $\mathscr{N}_{b, a}$; доказательство теорем 1.15, 1.12}

Рассматривается разностный оператор $\mathscr{D} \in \operatorname{End} \mathscr{F}(\mathbb{Z}, X)$, определенный формулой (1.8), который строится по ограниченной функции $U: \mathbb{Z} \rightarrow \operatorname{End} X$. Cоответствующее семейство эволюционных операторов $\mathscr{U}_{d}: \Delta_{\mathbb{Z}} \rightarrow$ End $X$ определено равенствами (1.11).

Пусть $m \geqslant 2, m \in \mathbb{N}$. По оператору $\mathscr{D}$ построим оператор $\mathscr{D}_{m} \in$ End $\mathscr{F}(\mathbb{Z}, X)$. Он строится по функции $U_{m}: \mathbb{Z} \rightarrow$ End $X$, где $U_{m}(n)=U(n m) U(n m-1) \cdots$ $U(n m-m+1)=\mathscr{U}(n m,(n-1) m+1), n \in \mathbb{Z}$, а $\mathscr{U}: \Delta_{\mathbb{Z}} \rightarrow$ End $X-$ семейство эволюционных операторов вида (1.11).

Оператор $\mathscr{D}_{m}$ определяется формулой

$$
\left(\mathscr{D}_{m} x\right)(x)=x(n)-U_{m}(n) x(n-1), \quad n \in \mathbb{Z}, \quad x \in \mathscr{F}(\mathbb{Z}, X) .
$$

Семейство $\mathscr{U}_{m}$ эволюционных операторов, отвечающих функции $U_{m}$, имеет вид $\mathscr{U}_{m}(n, k)=\mathscr{U}(m n, m k), k \leqslant n$.

Следующая теорема существенно используется при исследовании оператоpa $\mathscr{D}$.

ТеОрема 5.1. Имеет место равенство

$$
\operatorname{St}_{\text {inv }}\left(\mathscr{D}_{m}\right)=\operatorname{St}_{\text {inv }}(\mathscr{D})
$$

ЗАмЕчАниЕ 5.1. Пусть $\mathscr{F}\left(\mathbb{Z}_{-}, X\right)$ - однородное пространство последовательностей. Тогда однородным пространством последовательностей будет являться пространство

$$
\begin{aligned}
& \mathscr{F}\left(\mathbb{Z}_{+}, X\right)=\left\{y: \mathbb{Z}_{+} \rightarrow X \mid\right. y(k)=x(-k), k \in \mathbb{Z}_{+}, \\
&\text {где } \left.x \in \mathscr{F}\left(\mathbb{Z}_{-}, X\right) \text { с }\|y\|=\|x\|_{\mathscr{F}\left(\mathbb{Z}_{-}, X\right)}\right\} .
\end{aligned}
$$

Аналогично (с помощью отображения отражения) определяется однородное пространство функций $\mathscr{F}\left(\mathbb{R}_{+}, X\right)$ по однородному пространству функций $\mathscr{F}\left(\mathbb{R}_{-}, X\right)$. Построенные при определении пространств $\mathscr{F}\left(\mathbb{Z}_{+}, X\right), \mathscr{F}\left(\mathbb{R}_{+}, X\right)$ изометрии (отражения) обозначим через

$$
\mathscr{V}_{d}: \mathscr{F}\left(\mathbb{Z}_{-}, X\right) \rightarrow \mathscr{F}\left(\mathbb{Z}_{+}, X\right), \quad \mathscr{V}: \mathscr{F}\left(\mathbb{R}_{-}, X\right) \rightarrow \mathscr{F}\left(\mathbb{R}_{+}, X\right)
$$

Рассмотрим разностный оператор $\mathscr{D}_{E}^{-}: D\left(\mathscr{D}_{E}^{-}\right) \subset \mathscr{F}\left(\mathbb{Z}_{-}, X\right) \rightarrow \mathscr{F}\left(\mathbb{Z}_{-}, X\right)$ (см. формулу (1.8)). Непосредственный подсчет показывает, что имеет место равенство

$$
\widetilde{\mathscr{D}}_{E}^{+}=\mathscr{V}_{d} \mathscr{D}_{E}^{-} \mathscr{V}_{d}^{-1}
$$


где оператор $\widetilde{\mathscr{D}}_{E}^{+}: D\left(\widetilde{\mathscr{D}}_{E}^{+}\right) \subset \mathscr{F}\left(\mathbb{Z}_{+}, X\right) \rightarrow \mathscr{F}\left(\mathbb{Z}_{+}, X\right)$ имеет вид

$$
\begin{aligned}
\left(\widetilde{\mathscr{D}}_{E}^{+} x\right)(n) & =x(n)-U(n+1) x(n+1), \quad n \in \mathbb{Z}_{+}, \quad x \in D\left(\widetilde{\mathscr{D}}_{E}^{+}\right), \\
D\left(\widetilde{\mathscr{D}}_{E}^{+}\right) & =\left\{x \in \mathscr{F}\left(\mathbb{Z}_{+}, X\right) \mid x(0) \in E\right\} .
\end{aligned}
$$

Таким образом, изучение оператора $\mathscr{D}_{E}^{-}$может быть сведено к изучению оператора $\widetilde{\mathscr{D}}_{E}^{+}$(они подобны и, значит, $\left.\operatorname{St}_{\text {inv }}\left(\mathscr{D}_{E}^{-}\right)=\operatorname{St}_{\text {inv }}\left(\widetilde{\mathscr{D}}_{E}^{+}\right)\right)$. Верно и обратное заключение.

Свойства отношения $\mathscr{D}_{E}^{+}($см. формулу $(1.10))$ рассматривались в статьях [39]-[42]. Если $\mathscr{F}=\mathscr{F}(\mathbb{Z}, X)=\mathscr{F}_{c}(\mathbb{Z}, X)$, то сопряженное к $\mathscr{F}$ пространство $\mathscr{F}^{*}$ отождествляется с двойственным к нему пространством $\mathscr{F}^{\prime}=\mathscr{F}^{\prime}\left(\mathbb{Z}_{-}, X^{*}\right)$ (используется теорема 3.1 из [19]).

Предположим, что $\mathscr{F}=\mathscr{F}\left(\mathbb{Z}_{-}, X\right)=\mathscr{F}_{c}\left(\mathbb{Z}_{-}, X\right)$, что позволяет отождествить (в силу теоремы 3.1 из [19]) сопряженное к нему пространство $\mathscr{F}\left(\mathbb{Z}_{-}, X\right)^{*}$ с двойственным пространством $\mathscr{F}^{\prime}=\mathscr{F}^{\prime}\left(\mathbb{Z}_{-}, X^{*}\right)$. Из определения сопряженного отношения следует, что отношение $\left(\mathscr{D}_{E}^{-}\right)^{*} \in L R C\left(\mathscr{F}^{\prime}\right)$ состоит из пар $(\eta, \xi) \in \mathscr{F}^{\prime} \times \mathscr{F}^{\prime}$ таких, что

$$
\langle y, \eta\rangle=\sum_{n \leqslant 0}\langle y(n), \eta(n)\rangle=\sum_{n \leqslant 0}\langle x(n), \xi(n)\rangle=\langle x, \xi\rangle
$$

для всех $x \in \mathscr{F}$ с $x(0) \in E$, для которых $\mathscr{D}_{E}^{-} x=y$. Поэтому отношение $\left(\mathscr{D}_{E}^{-}\right)^{*}$ определяется равенством

$$
\begin{array}{r}
\left(\mathscr{D}_{E}^{-}\right)^{*}=\left\{(\eta, \xi) \in \mathscr{F}^{\prime} \times \mathscr{F}^{\prime} \mid \xi(n)=\eta(n)-U(n+1)^{*} \eta(n+1),\right. \\
\left.n \leqslant-1, \xi(0)+\eta_{0}, \eta_{0} \in E^{\perp}=F\right\} .
\end{array}
$$

Ясно, что $D\left(\left(\mathscr{D}_{E}^{-}\right)^{*}\right)=\mathscr{F}^{\prime}$ и $\left(\mathscr{D}_{E}^{-}\right)^{*} 0=\left\{\xi \in \mathscr{F}^{\prime} \mid \xi(0) \in E^{\perp}, \xi(k)=0, k \leqslant-1\right\}$. Оператор $\left(\mathscr{D}_{X}^{-}\right)^{*}$ принадлежит End $\mathscr{F}^{\prime}$. Таким же образом устанавливается, что сопряженное отношение к оператору $\widetilde{\mathscr{D}}_{E}^{+}$(подобному оператору $\mathscr{D}_{E}^{-}$) из замечания 5.3 ниже имеет вид

$$
\begin{gathered}
\left(\widetilde{\mathscr{D}}_{E}^{+}\right)^{*}=\left\{(\eta, \xi) \in \mathscr{F}^{\prime}\left(\mathbb{Z}_{+}, X^{*}\right) \times \mathscr{F}^{\prime}\left(\mathbb{Z}_{+}, X^{*}\right) \mid \xi(n)=\eta(n)-U(-n+1)^{*} \eta(n-1),\right. \\
\left.n \geqslant 1, \xi(0)=\eta(0)+\eta_{0}, \eta_{0} \in E^{\perp}\right\} .
\end{gathered}
$$

ЗАмечАниЕ 5.2. Отношения $\left(\mathscr{D}_{E}^{-}\right)^{*},\left(\widetilde{D}_{E}^{+}\right)^{*}$ подобны и связаны равенством

$$
\left(\widetilde{\mathscr{D}}_{E}^{+}\right)^{*}=\widetilde{\mathscr{V}}_{d}\left(\mathscr{D}_{E}^{-}\right)^{*} \widetilde{\mathscr{V}}_{d}^{-1},
$$

где оператор $\widetilde{\mathscr{V}}_{d}: \mathscr{F}^{\prime}\left(\mathbb{Z}_{-}, X^{*}\right) \rightarrow \mathscr{F}^{\prime}\left(\mathbb{Z}_{+}, X^{*}\right)$ имеет вид $\left(\widetilde{\mathscr{V}}_{d} \xi\right)(n)=\xi(-n), n \leqslant 0$, $\xi \in \mathscr{F}^{\prime}\left(\mathbb{Z}_{-}, X^{*}\right)$. Важно отметить, что отношение $\left(\widetilde{\mathscr{D}}_{E}^{+}\right)^{*} \in \operatorname{LRC}\left(\mathscr{F}^{\prime}\left(\mathbb{Z}_{+}, X^{*}\right)\right)$ совпадает с отношением $\mathscr{D}_{E^{\perp}}^{+}$, построенным по функции $\widetilde{U}(n)=U(-n+1)^{*}$, $n \in \mathbb{Z}_{+}$, т. е. относится к классу изученных нами в разделе 4 и статье [19] разностных отношений. Итак, появляется возможность использования полученных там результатов.

Таким образом, для отношения $\mathscr{D}_{E}^{-}$имеют место аналоги всех результатов, полученных в [19] и в разделе 3 настоящей статьи. 
5.1. Доказательство теоремы 1.15. В статье [43] (см. также [27]) для изучения оператора $\mathscr{D}$ использовались два разностных оператора $\mathscr{D}^{-}=D_{X}^{-,-1} \in$ End $\mathscr{F}\left(\mathbb{Z}_{-} \backslash\{0\}, X\right), \mathscr{D}^{+}=\mathscr{D}_{\{0\}}^{+} \in$ End $\mathscr{F}\left(\mathbb{Z}_{+}, X\right)$, которые определяются равенствами

$$
\begin{aligned}
& \left(\mathscr{D}^{-} x\right)(n)=x(n)-U(n) x(n-1), \quad n \leqslant-1, \quad x \in \mathscr{F}_{-}=\mathscr{F}\left(\mathbb{Z}_{-} \backslash\{0\}, X\right), \\
& \left(\mathscr{D}^{+} x\right)(n)=\left(\mathscr{D}_{\{0\}}^{+} x\right)(n)= \begin{cases}x(0), & n=0, \\
x(n)-U(n) x(n-1), & n \geqslant 1, x \in \mathscr{F}_{+}=\mathscr{F}\left(\mathbb{Z}_{+}, X\right),\end{cases}
\end{aligned}
$$

а также оператор

$$
\mathscr{D}_{0}: \mathscr{F}_{-} \rightarrow \mathscr{F}_{+}, \quad\left(\mathscr{D}_{0} x\right)(n)=\left\{\begin{array}{ll}
U(0) x(-1), & n=0, \\
0, & n \geqslant 1,
\end{array} \quad x \in \mathscr{F}_{-} .\right.
$$

Однородные пространства $\mathscr{F}$ - и $\mathscr{F}+$ получены из $\mathscr{F}(\mathbb{Z}, X)$ сужением последовательностей из $\mathscr{F}(\mathbb{Z}, X)$ на $\mathbb{Z}_{-} \backslash\{0\}$ и $\mathbb{Z}_{+}$соответственно. Таким образом, $\mathscr{F}=\mathscr{F}(\mathbb{Z}, X)=\mathscr{F}_{-} \times \mathscr{F}_{+}$. Пространства $\mathscr{F}_{-}, \mathscr{F}_{+}$удобно рассматривать также как подпространства из $\mathscr{F}$, и тогда $\mathscr{F}=\mathscr{F}_{-} \oplus \mathscr{F}_{+}$. Относительно любого из представлений пространства $\mathscr{F}$ оператор $\mathscr{D}$ задается матрицей

$$
\left(\begin{array}{cc}
\mathscr{D}^{-} & 0 \\
\mathscr{D}_{0} & \mathscr{D}^{+}
\end{array}\right)
$$

Далее используется следующая лемма из [44; р. 23], относящаяся к операторам, имеющим матричное представление (5.2).

Лемма 5.1. Пусть банахово пространство $\mathscr{X}$ есть прямая сумма $\mathscr{X}_{1} \oplus \mathscr{X}_{2}$ двух замкнутых подпространств $\mathscr{X}_{1}, \mathscr{X}_{2}$ и оператор $A \in$ End $\mathscr{X}$ допускает матричное представление

$$
\left(\begin{array}{cc}
A_{11} & 0 \\
A_{21} & A_{22}
\end{array}\right), \quad A_{11} \in \text { End } \mathscr{X}_{1}, \quad A_{21} \in \operatorname{Hom}\left(\mathscr{X}_{1}, \mathscr{X}_{2}\right), \quad A_{22} \in \text { End } \mathscr{X}_{2} .
$$

Тогда оператор А фредгольмов тогда и только тогда, когда выполнены следующие условия:

1) $\operatorname{Im} A_{11}$ - замкнутое подпространство из $\mathscr{X}_{1}$ и codim $\operatorname{Im} A_{11}<\infty$;

2) $\operatorname{Im} A_{22}$ - замкнутое подпространство в $\mathscr{X}_{2} u \operatorname{dim} \operatorname{Ker} A_{22}<\infty$;

3) подпространство $\mathscr{X}_{1}^{0}=\left\{x \in \mathscr{X}_{1} \mid x \in \operatorname{Ker} A_{11} u A_{21} x \in \operatorname{Im} A_{22}\right\}$ uмеeт конечную размерность в $\mathscr{X}_{1}$;

4) подпространство $\mathscr{X}_{2}^{0}=\operatorname{Im} A_{22}+A_{21}\left(\operatorname{Ker} A_{11}\right)$ имеет конечную коразмерность в $\mathscr{X}_{2}$.

Если выполнены свойства 1)-4), то

$\operatorname{dim} \operatorname{Ker} A=\operatorname{dim} \operatorname{Ker} A_{22}+\operatorname{dim} \mathscr{X}_{1}^{0}, \quad \operatorname{codim} \operatorname{Im} A=\operatorname{codim} \operatorname{Im} A_{11}+\operatorname{codim} \mathscr{X}_{2}^{0}$.

ДоказАтельство теоремы 1.15. Пусть оператор $\mathscr{D}$ фредгольмов. Рассмотрим подпространство $E=U(0) X_{0}(-1)$, где $X_{0}(-1)=\left\{x(-1) \mid x \in \operatorname{Ker} \mathscr{D}_{-}\right\}$. Отметим замкнутость подпространства $\mathscr{D}\left(\operatorname{Ker} \mathscr{D}_{-}\right) \cap \mathscr{F}_{+}(\mathbb{Z}, X)$. Оно состоит из последовательностей $y \in \mathscr{F}_{+}(\mathbb{Z}, X)$, представимых в виде: $y(k)=0, k \geqslant 1$, 
$y(0)=-U(0) x(-1), x(-1) \in X_{0}(-1)$. Следовательно, $E$ - замкнутое подпространство из $X$. Непосредственно из определения подпространства $E$ следует равенство

$$
\operatorname{Im} \mathscr{D}_{+}+\mathscr{D}_{0}\left(\operatorname{Ker} \mathscr{D}_{-}\right)=\operatorname{Im} \mathscr{D}_{E}^{+} .
$$

Теперь из свойства 4) в лемме 5.1 получаем, что образ $\operatorname{Im} \mathscr{D}_{E}^{+}$отношения $\mathscr{D}_{E}^{+} \in L R C\left(\mathscr{F}_{+}\right)$имеет конечную коразмерность в $\mathscr{F}_{+}$.

Докажем конечномерность ядра $\operatorname{Ker} \mathscr{D}_{E}^{+}$отношения $\mathscr{D}_{E}^{+}$. Пусть $\widetilde{x} \in \operatorname{Ker} \mathscr{D}_{E}^{+}$. Следовательно, $\widetilde{x}(0) \in E$ и $\widetilde{x}(n)=\mathscr{U}(n, 0) x_{0}, n \geqslant 0$. Из определения подпространства $E$ следует, что вектор $\widetilde{x}(0)$ представим в виде $\widetilde{x}(0)=U(0) x_{0}$, где $x_{0} \in X_{0}(-1)$. Поэтому существует последовательность $x \in \mathscr{F}$ - такая, что $x \in \operatorname{Ker} \mathscr{D}_{-}$и $x(-1)=x_{0}$.

Последовательность $\widetilde{x}$ продолжим на $\mathbb{Z}$ - до последовательности $y \in \mathscr{F}(\mathbb{Z}, X)$, положив $y(n)=\widetilde{x}(n), n \geqslant 0$, и $y(n)=x(n), n \leqslant-1$. Из ее определения следует, что $y \in \operatorname{Ker} \mathscr{D}$. Следовательно, $\operatorname{dim} \operatorname{Ker} \mathscr{D}_{E}^{+} \leqslant \operatorname{dim} \operatorname{Ker} \mathscr{D}<\infty$.

Итак, установлена фредгольмовость отношения $\mathscr{D}_{E}^{+}$. Следовательно, существует $m \in \mathbb{Z}_{+}$такое, что семейство $\mathscr{U}$ допускает экспоненциальную дихотомию на $\mathbb{Z}_{m,+}$ с некоторой расщепляющей парой $P_{+}, Q_{+}: \mathbb{Z}_{m,+} \rightarrow$ End $X$.

Переходя, если необходимо, к подобному оператору $S(-m) \mathscr{D} S(m)$, определяемому операторной функцией $U_{m}(n)=U(n-m), n \in \mathbb{Z}$ (см. замечание 5.3 в п. 5.2), далее будем считать, что $m=0$.

Докажем фредгольмовость оператора $\mathscr{D}_{F}^{-,(-1)}=\mathscr{D}_{F,-}$, определенного в $\mathscr{F}_{-}$ формулой (1.9), где $E=F=U(0)^{-1} \operatorname{Im} P_{+}(0)$, с областью определения

$$
D\left(\mathscr{D}_{F,-}\right)=\left\{x \in \mathscr{F}_{-} \mid x(-1) \in U(0)^{-1}\left(\operatorname{Im} P_{+}(0)\right)=F\right\} .
$$

Рассмотрим подпространство $\mathscr{F}_{-}^{0}$ из $\mathscr{F}(\mathbb{Z}, X)$ вида

$$
\mathscr{F}_{-}^{0}=\left\{x \in \mathscr{F}(\mathbb{Z}, X) \mid x(k)=0, k \geqslant 1, x(0) \in \operatorname{Ker} Q_{+}(0)=\operatorname{Im} P_{+}(0)\right\} .
$$

Докажем равенство $\operatorname{Im} \mathscr{D}_{F,-}=\{\widetilde{y} \in \mathscr{F} \mid \widetilde{y}$ - сужение некоторой последовательности $y$ из $\left.\operatorname{Im} \mathscr{D} \cap \mathscr{F}_{-}^{0}\right\}$.

Пусть $y \in \operatorname{Im} \mathscr{D} \cap \mathscr{F}_{-}^{0}$. Тогда существует последовательность $x \in \mathscr{F}(\mathbb{Z}, X)$ такая, что

$y(n)=x(n)-U(n) x(n-1), \quad n \leqslant 0 ; \quad y(n)=0, \quad n \geqslant 1 ; \quad y(0) \in \operatorname{Im} P_{+}(0)$.

Тогда $P_{+}(0) y(0)=y(0)=P_{+}(0) x(0)-P_{+}(0) U(0) x(-1)$. Из равенств $x(n)=$ $\mathscr{U}(n, 0) x(0), n \geqslant 0$, и наличия у $\mathscr{U}$ экспоненциальной дихотомии на $\mathbb{Z}_{+}$следует, что $x(0) \in \operatorname{Im} P_{+}(0)$. Поскольку $y(0) \in \operatorname{Im} P_{+}(0)$, то из равенства $x(0)-y(0)=$ $U(0) x(-1)$ получаем, что $U(0) x(-1) \in \operatorname{Im} P_{+}(0)$, т. е. $x(-1) \in F$.

Пусть теперь $y_{-} \in \operatorname{Im} \mathscr{D}_{F,-}$. Тогда существует последовательность $x_{-} \in \mathscr{F}_{-}$, для которой $x_{-}(-1) \in F$ и имеют место равенства $y_{-}(n)=x_{-}(n)-U(n) x_{-}(n-$ $1), n \leqslant-1$. Положим $x(n)=x_{-}(n), n \leqslant-1$, и $x(n)=U(n) x(n-1), n \geqslant 0$. Ясно, что $\mathscr{D} x=y \in \mathscr{F}(\mathbb{Z}, X)$, где сужением последовательности на $\mathbb{Z}-\backslash\{0\}$ является последовательность $y_{-}$.

Из доказанного равенства и замечания 5.1 следует, что $\operatorname{Im} \mathscr{D}_{F,-}$ имеет конечную коразмерность в $\mathscr{F}_{-}$, не превосходящую codim $\operatorname{Im} \mathscr{D}$. 
Докажем конечномерность ядра $\operatorname{Ker} \mathscr{D}_{F,-}$ рассматриваемого оператора $\mathscr{D}_{F,-}$. Пусть $x_{0} \in \operatorname{Ker} \mathscr{D}_{F,-}$. Тогда имеют место следующие равенства: $x_{0}(-1)=$ $\mathscr{U}(0, n) x_{0}(n), n \leqslant-1$, причем $U(0) x_{0}(-1) \in \operatorname{Im} P_{+}(0)$. Рассмотрим последовательность $\widetilde{x}_{0} \in \mathscr{F}(\mathbb{Z}, X)$, для которой $\widetilde{x}_{0}(n)=x_{0}(n), n \leqslant-1$, и $\widetilde{x}_{0}(n)=$ $\mathscr{U}(n, 0) U(0) x(-1), n \geqslant 0$. Поскольку $U(0) x(-1) \in \operatorname{Im} P_{+}(0)$, то $\widetilde{x}_{0}$ экспоненциально убывает при $n \rightarrow \infty$. Поэтому $\widetilde{x}_{0} \in \mathscr{F}(\mathbb{Z}, X)$. Из ее построения следует, что $\widetilde{x}_{0} \in \operatorname{Ker} \mathscr{D}$. Из доказанного получаем, что $\operatorname{dim} \operatorname{Ker} \mathscr{D}_{F,-} \leqslant \operatorname{dim} \operatorname{Ker} \mathscr{D}<\infty$.

Итак, установлена фредгольмовость оператора $\mathscr{D}_{F,-}$. Теперь из замечания 5.1 следует, что семейство $\mathscr{U}$ несингулярно на $-\infty$, т. е. доказано существование чисел $a \leqslant b, a, b \in \mathbb{Z}$, таких, что семейство $\mathscr{U}$ допускает экспоненциальную дихотомию на $\mathbb{Z}_{-, a}$ и $\mathbb{Z}_{b,+}$ с расщепляющими парами $P_{-}, Q_{-}: \mathbb{Z}_{-, a} \rightarrow$ End $X$ и $P_{+}, Q_{+}: \mathbb{Z}_{b,+} \rightarrow$ End $X$ проекторнозначных функций. В этом случае в статье [43] для оператора $\mathscr{D}$, действующего в любом из пространств $l^{p}(\mathbb{Z}, X)$, $p \in[1, \infty]$, и $c_{0}(\mathbb{Z}, X)$, была доказана фредгольмовость узлового оператора $\mathscr{N}_{b, a}: \operatorname{Im} Q_{-}(a) \rightarrow \operatorname{Im} Q_{+}(a)$, определенного формулой (1.20). Доказательство, приведенное в [43], проходит без изменений для сужения оператора $\mathscr{D}$ на подпространство $\mathscr{F}_{c}(\mathbb{Z}, X)$, которое является инвариантным для $\mathscr{D}$. Фредгольмовость оператора $\mathscr{N}_{b, a}$ будет также следовать из теоремы 1.12. Из статьи [43] и теоремы 1.12 будет следовать достаточность условия доказываемой теоремы. Достаточность условий теоремы также следует из теоремы 5.3 ниже. Теорема 1.15 доказана.

5.2. Доказательство теоремы 1.12. Далее (до конца этого раздела) считаются выполненными условия несингулярности семейства $\mathscr{U}_{d}: \Delta_{\mathbb{Z}} \rightarrow$ End $X$ на $\pm \infty$, т. е. считается выполненным следующее предположение.

ПреДПОЛОЖенИЕ 5.1. Существуют целье числа $a, b, a \leqslant b$, такие, что семейство $\mathscr{U}_{d}$ допускает экспоненциальную дихотомию на множествах $\mathbb{Z}_{-, a}$ $u \mathbb{Z}_{b,+}$ с расщепляющими парами проекторнозначных функций $P_{-}, Q_{-}: \mathbb{Z}_{-, a} \rightarrow$ End $X u P_{+}, Q_{+}: \mathbb{Z}_{b,+} \rightarrow$ End $X$.

Рассматривается узловой оператор

$$
\mathscr{N}_{b, a}: \operatorname{Im} Q_{-}(a) \rightarrow \operatorname{Im} Q_{+}(b), \quad \mathscr{N}_{a, b} x=Q_{+}(b) \mathscr{U}(b, a) x, \quad x \in \operatorname{Im} Q_{-}(a) .
$$

Приводимое далее замечание показывает, что можно ограничиться случаем $a=$ $-1, b=0$.

ЗАмЕчАниЕ 5.3. Оператор $(S(b) \mathscr{D} S(-b) x)(n)=x(n)-U(n+b) x(n-1)$, $n \in \mathbb{Z}, x \in \mathscr{F}(\mathbb{Z}, X)$, подобен оператору $\mathscr{D}$, и поэтому

$$
\operatorname{St}_{\text {inv }}(\mathscr{D})=\operatorname{St}_{\text {inv }}(S(b) \mathscr{D} S(-b)) .
$$

Семейство эволюционных операторов $\mathscr{U}_{b}$, которое строится по функциям $U_{b}(n)=U(n+b), n \in \mathbb{Z}, b \in \mathbb{Z}$, допускает экспоненциальную дихотомию на $\mathbb{Z}_{-}$ и $\mathbb{Z}_{m,+}$, где $m=b-a$, с расщепляющими парами $P_{ \pm}(n+b), Q_{ \pm}(n+b), n \in \mathbb{Z}$. Теперь к разностному оператору $S(b) \mathscr{D} S(-b)$ применим теорему 5.1 , из которой будет следовать, что он (и, следовательно, оператор $\mathscr{D})$ имеет одинаковое множество состояний обратимости с оператором $\widetilde{\mathscr{D}} \in \operatorname{End} \mathscr{F}(\mathbb{Z}, X)$, который 
строится с помощью функций $\widetilde{U}_{m}(n)=U(m n+b) \cdots U(m n-m+b+1)=$ $\mathscr{U}(m n+b, m(n-1)+b)$. Соответствующее семейство эволюционных операторов $\widetilde{\mathscr{U}_{d}}$ имеет вид

$$
\widetilde{\mathscr{U}_{d}}(n, k)=\mathscr{U}_{d}((b-a) n+b,(b-a) k+b), \quad k \leqslant n, \quad k, n \in \mathbb{Z} .
$$

Семейство (5.3) допускает экспоненциальную дихотомию на $\mathbb{Z}_{-,-1}=\mathbb{Z}_{-} \backslash\{0\}$, $\mathbb{Z}_{+}$с расщепляющими парами проекторнозначных функций

$$
\begin{aligned}
& \widetilde{P}_{-}, \widetilde{Q}_{-}: \mathbb{Z}_{-} \backslash\{0\} \rightarrow \text { End } X, \quad \widetilde{P}_{+}, \widetilde{Q}_{+}: \mathbb{Z}_{+} \rightarrow \text { End } X, \\
& \widetilde{P}_{-}(n)=P_{-}((b-a) n+b), \quad n \leqslant-1, \quad \widetilde{P}_{+}(n)=P_{+}((b-a) n+b), \quad n \geqslant 1, \\
& \widetilde{Q}_{-}=I-\widetilde{P}_{-}, \quad \widetilde{Q}_{+}=I-\widetilde{P}_{+} .
\end{aligned}
$$

Узловой оператор, определяемый семейством $\widetilde{\mathscr{U}}: \Delta_{\mathbb{Z}} \rightarrow \operatorname{End} X$, имеет вид

$$
\begin{gathered}
\widetilde{\mathscr{N}}: \operatorname{Im} \widetilde{Q}_{-}(-1) \rightarrow \operatorname{Im} \widetilde{Q}_{+}(0), \\
\widetilde{\mathscr{N} x}=\widetilde{Q}_{+}(0) \widetilde{\mathscr{U}_{d}}(0,-1) x=Q_{+}(b) \mathscr{U}_{d}(b, a) x=\mathscr{N}_{b, a}, \quad x \in \operatorname{Im} Q_{-}(a)=\operatorname{Im} \widetilde{Q}_{-}(0),
\end{gathered}
$$

т. е. совпадает с узловым оператором $\mathscr{N}_{b, a}$. Таким образом, $\operatorname{St}_{\text {inv }}(\mathscr{D})=\operatorname{St}_{\text {inv }}(\widetilde{\mathscr{D}})$ и $\mathscr{N}_{b, a}=\widetilde{\mathscr{N}}$.

Из сделанного замечания следует, что без ограничения общности можно считать, что рассматриваемое семейство эволюционных операторов $\mathscr{U}$ допускает экспоненциальную дихотомию на множествах $\mathbb{Z}_{-} \backslash\{0\}, \mathbb{Z}_{+}$с расщепляющими парами $P_{-}, Q_{-}: \mathbb{Z}_{-} \backslash\{0\}, P_{+}, Q_{+}: \mathbb{Z}_{+} \rightarrow$ End $X$ и узловым оператором

$$
\mathscr{N}: \operatorname{Im} Q_{-}(-1) \rightarrow \operatorname{Im} Q_{+}(0), \quad \mathscr{N} x=Q_{+}(0) U(0) x, \quad x \in Q_{-}(-1),
$$

где учитывается, что $\mathscr{U}(0,-1)=U(0)$.

Именно такие условия на $\mathscr{U}$ считались выполненными в статье [43] при исследовании разностного оператора $\mathscr{D} \in \operatorname{End} l^{p}(\mathbb{Z}, X), p \in[1, \infty]$, которые были получены с использованием узлового оператора. Поскольку доказательства большинства утверждений из [43] верны и для оператора $\mathscr{D}$, действующего в однородном пространстве, то некоторые из этих утверждений будут использоваться в дальнейшем.

Tеорема 5.2. Пусть для семейства $\mathscr{U}_{d}: \Delta_{\mathbb{Z}} \rightarrow$ End $X$ выполнено предположение 1.1 с $Q^{ \pm}=0$. Тогда оператор $\mathscr{D}$ непрерывно обратим.

ДокАЗАТЕЛЬство. В силу сделанного замечания 5.1 достаточно рассмотреть случай, когда $a=-1, b=0$. Матрицу (5.1) оператора $\mathscr{D} \in \mathscr{F}(\mathbb{Z}, X)$ представим в виде

$$
\left(\begin{array}{cc}
\mathscr{D}^{-} & 0 \\
\mathscr{D}_{0} & \mathscr{D}^{+}
\end{array}\right)=\left(\begin{array}{cc}
\mathscr{D}^{-} & 0 \\
0 & I
\end{array}\right)\left(\begin{array}{cc}
I & 0 \\
\mathscr{D}_{0} & I
\end{array}\right)\left(\begin{array}{cc}
I & 0 \\
0 & \mathscr{D}^{+}
\end{array}\right) .
$$

При такой факторизации оператора $\mathscr{D}$ каждый из сомножителей (в силу условий $\left.Q_{-}=0, Q_{+}=0\right)$ является непрерывно обратимым оператором. Следовательно, таким является оператор $\mathscr{D}$. Теорема доказана. 
Рассмотрим линейный оператор $K: \operatorname{Ker} \mathscr{N} \rightarrow \operatorname{Ker} \mathscr{D}$ вида

$$
\left(K x_{0}\right)(n)=\left\{\begin{array}{ll}
\mathscr{U}_{d}(n,-1) x_{0}=\mathscr{U}_{d}(n,-1) Q_{-}(-1) x_{0}, & n \leqslant-1, \\
\mathscr{U}_{d}(n, 0) U(0) x_{0}, & n \geqslant 0,
\end{array} \quad x_{0} \in \operatorname{Ker} \mathscr{N} .\right.
$$

Теорема 5.3. Оператор $K$ корректно определен, ограничен и осуществляет изоморфизм подпространств $\operatorname{Ker} \mathscr{N}, \operatorname{Ker} \mathscr{D}$.

Сформулированная теорема следует из [43; теорема 4]. При этом отметим, что если $x_{0} \in \operatorname{Ker} \mathscr{D}$, то из наличия экспоненциальной дихотомии для $\mathscr{U}$ на $\mathbb{Z}_{-} \backslash\{0\}$ и $\mathbb{Z}_{+}$следует существование постоянных $M_{0}>0, q_{0} \in(0,1)$ таких, что $\left\|x_{0}(n)\right\| \leqslant M_{0} q_{0}^{|n|}, n \in \mathbb{Z}$. Следовательно, ядро Ker $\mathscr{D}$ оператора не зависит от выбора однородного пространства $\mathscr{F}(\mathbb{Z}, X)$.

Лемма 5.2. Пусть подпространство $\operatorname{Ker} \mathscr{N} \subset \operatorname{Im} Q_{-}(-1)$ дополняемо $в$ $\operatorname{Im} Q_{-}(-1)$, причем

$$
\operatorname{Im} Q_{-}(-1)=\operatorname{Ker} \mathscr{N} \oplus X_{1},
$$

где $X_{1}$ - замкнутое подпространство из $\operatorname{Im} Q_{-}(-1)$. Тогда

$$
\mathscr{F}(\mathbb{Z}, X)=\operatorname{Ker} \mathscr{D} \oplus \mathscr{F} 1,
$$

где $\mathscr{F}_{1}=\left\{x \in \mathscr{F} \mid x(-1) \in \widetilde{X}_{1}\right\}, \widetilde{X}_{1}=X_{1} \oplus \operatorname{Im} P_{-}(-1)$.

ДокАзАТЕльство. Достаточно заметить, что представление (5.6) следует из (5.5) и представления (5.4) последовательностей из Ker $\mathscr{D}$.

Введем в рассмотрение оператор $\mathscr{A}: \mathscr{F}(\mathbb{Z}, X) \rightarrow \mathscr{F}(\mathbb{Z}, X)$ вида

$$
(\mathscr{A} x)(n)=\left\{\begin{array}{ll}
\mathscr{U}_{d}(n,-1) Q_{-}(-1) x(-1), & n \leqslant-1, \\
\mathscr{U}_{d}(n, 0) P_{+}(0) U(0) Q_{-}(-1) x(-1), & n \geqslant 0,
\end{array} \quad x \in \mathscr{F}(\mathbb{Z}, X) .\right.
$$

Из его определения следует, что $\mathscr{A} \in \operatorname{End} \mathscr{F}(\mathbb{Z}, X)$ и $\mathscr{A}^{2}=\mathscr{A}$, т. е. $\mathscr{A}-$ проектор. В частности, $\operatorname{Im} \mathscr{A}$ - замкнутое подпространство из $\mathscr{F}(\mathbb{Z}, X)$.

Лемма 5.3. Имеет место равенство $\mathscr{A} x=x$ для любого $x \in \operatorname{Ker} \mathscr{D}$.

ДоказАтеЛьство. Пусть $x \in \operatorname{Ker} \mathscr{D}$. Тогда из (5.4) следует, что

$$
x(n)= \begin{cases}\mathscr{U}_{d}(n,-1) Q_{-}(-1) x_{0}, & n \leqslant-1, \\ \mathscr{U}_{d}(n, 0) U(0) x_{0}, & n \geqslant 0\end{cases}
$$

где $x_{0} \in \operatorname{Ker} \mathscr{N}$ и

$$
\begin{aligned}
& (\mathscr{A} x)(n)=\mathscr{U}_{d}(n,-1) Q_{-}(-1) x_{0}, \\
& n \leqslant-1 \text {, } \\
& (\mathscr{A} x)(n)=\mathscr{U}_{d}(n, 0) P_{+}(0) U(0) Q_{-}(-1) x_{0}=\mathscr{U}_{d}(n, 0) U(0) Q_{-}(-1) x_{0}, \quad n \geqslant 0 .
\end{aligned}
$$

Следовательно, $\mathscr{A} x=x$. Лемма доказана. 
Пусть пространство $\mathscr{F}(\mathbb{Z}, X)$ допускает разложение

$$
\mathscr{F}(\mathbb{Z}, X)=\operatorname{Ker} \mathscr{D} \oplus \mathscr{F}_{1},
$$

где $\mathscr{F}_{1}$ - замкнутое подпространство из $\mathscr{F}(\mathbb{Z}, X)$. Тогда в соответствии с этим разложением из леммы 5.2 получаем представление

$$
\operatorname{Im} \mathscr{A}=\operatorname{Ker} \mathscr{D} \oplus \mathscr{F}_{0} .
$$

Поскольку $\mathscr{A} \operatorname{Ker} \mathscr{D}=\operatorname{Ker} \mathscr{D}$, то и $\mathscr{F}_{0}-$ замкнутое подпространство из $\operatorname{Im} \mathscr{A}$. Рассмотрим оператор $A_{0}: \operatorname{Im} \mathscr{A} \rightarrow \operatorname{Im} Q_{-}(-1)$, заданный формулой $A_{0} x=$ $x(-1) \in \operatorname{Im} Q_{-}(-1), x \in \operatorname{Im} \mathscr{A}$. Из вида $\operatorname{Im} \mathscr{A}$ следует, что $A_{0}$ является изоморфизмом. Применяя к обеим частям равенства (5.8) оператор $A_{0}^{-1}$, получим разложение

$$
\operatorname{Im} Q_{-}(-1)=\operatorname{Ker} \mathscr{N} \oplus A_{0}^{-1}\left(\mathscr{F}_{0}\right)
$$

Итак, доказана следующая лемма.

Лемма 5.4. Пусть ядро оператора $\mathscr{D}$ дополняемо в $\mathscr{F}(\mathbb{Z}, X)$ (имеет место разложение (5.7)). Тогда ядро узлового оператора $\mathscr{N}$ дополняемо в $\operatorname{Im} Q_{-}(-1)$ и верно разложение (5.9).

Вернемся к матричному представлению (5.1) оператора $\mathscr{D}$ относительно разложения $\mathscr{F}(\mathbb{Z}, X)=\mathscr{F}-\oplus \mathscr{F}_{+}$. Из этого представления следует, что верна (см. доказательство теоремы 1.15 и теорему 5.1) следующая лемма.

Лемма 5.5. Имеют место равенства

$$
\operatorname{Im} \mathscr{D}_{-}=\operatorname{Im} \mathscr{D} \cap \mathscr{F}_{-},
$$

$\operatorname{Im} \mathscr{D}_{+}+\mathscr{D}_{0}\left(\operatorname{Ker} \mathscr{D}_{-}\right)=\operatorname{Im} \mathscr{D} \cap \mathscr{F}_{+}=\operatorname{Im} \mathscr{D}_{E}^{+} \in L R C\left(\mathscr{F}_{+}\right)$,

где $E=U(0)\left(\operatorname{Im} Q_{-}(-1)\right)$. Подпространство $\operatorname{Im} \mathscr{D}$ замкнуто тогда и только тогда, когда замкнут образ $\operatorname{Im} \mathscr{D}_{E}^{+}$отношения $\mathscr{D}_{E}^{+}$.

Отметим, что в условиях леммы 5.5 отношение $\mathscr{D}_{E}^{+}$определяется равенствами (1.10), где $a=0$, и $E-$ не обязательно замкнутое подпространство из $X$.

Лемма 5.6. Если $\operatorname{Im} \mathscr{D}_{E}^{+}-$замкнутое подпространство из $\mathscr{F}_{+}$, то подпространство $E \subset X$ замкнуто.

ДокАзАтельство. Пусть $\left(x_{n}\right)$ - сходящаяся к вектору $x_{0} \in X$ последовательность векторов из $E$. Тогда последовательность $\widetilde{x}_{n} \in \mathscr{F}_{+}, \widetilde{x}_{n}(0)=x_{n}$, $\widetilde{x}_{n}(k)=0, k \geqslant 1$, принадлежит $\operatorname{Im} \mathscr{D}_{E}^{+}$и является сходящейся к $\widetilde{x}_{0} \in \mathscr{F}_{+}$, $\widetilde{x}_{0}(0)=x_{0}, \widetilde{x}_{0}(k)=0, k \geqslant 1$. Следовательно, $\widetilde{x}_{0} \in \operatorname{Im} \mathscr{D}_{E}^{+}$, и поэтому $x_{0} \in E$. Лемма доказана.

Лемма 5.7. Имеет место следующее свойство:

$$
\overline{\operatorname{Im} \mathscr{D}}=\operatorname{Im} \mathscr{D} \quad \Leftrightarrow \quad \overline{\operatorname{Im} \mathscr{N}}=\operatorname{Im} \mathscr{N} .
$$


ДокАЗАТЕЛЬство. Пусть $\overline{\operatorname{Im} \mathscr{D}}=\operatorname{Im} \mathscr{D}$. Тогда из леммы 5.5 получаем замкнутость подпространства $\operatorname{Im} \mathscr{D}_{E}^{+}$в $\mathscr{F}_{+}$, а из леммы 5.6 следует замкнутость $E$ в $X$. Поскольку семейство $\mathscr{U}$ допускает экспоненциальную дихотомию на $\mathbb{Z}_{+}$, то из теоремы 1.11 вытекает, что $\operatorname{St}_{\text {inv }}\left(\mathscr{D}_{E}^{+}\right)=\operatorname{St}_{\text {reg }}\left(\operatorname{Im} P_{+}(0), E\right)$. Поэтому замкнуто подпространство $\operatorname{Im} P_{+}(0)+E$ (см. свойство 4) из определения 1.4). Замкнутость $\operatorname{Im} \mathscr{N}$ следует из представления подпространства $\operatorname{Im} P_{+}(0)+E$ в виде

$$
\begin{aligned}
\operatorname{Im} P_{+}(0)+E & =\operatorname{Im} P_{+}(0)+\operatorname{Im}\left(U(0) Q_{-}(-1)\right) \\
& =\operatorname{Im} P_{+}(0)+\operatorname{Im}\left(P_{+}(0) U(0) Q_{-}(-1)\right)+\operatorname{Im} \mathscr{N}=\operatorname{Im} P_{+}(0) \oplus \operatorname{Im} \mathscr{N}
\end{aligned}
$$

Обратно, пусть $\overline{\operatorname{Im} \mathscr{N}}=\operatorname{Im} \mathscr{N}$. Тогда $\operatorname{Im} P_{+}(0)+E-$ замкнутое в $X$ подпространство в силу той же теоремы 1.11. Следовательно, $\overline{\operatorname{Im} \mathscr{D}_{E}}=\operatorname{Im} \mathscr{D}_{E}=$ $\operatorname{Im} \mathscr{D} \cap \mathscr{F}_{+}$. Поскольку $\operatorname{Im} \mathscr{D} \cap \mathscr{F}_{-}=\mathscr{F}_{-}$, то замкнутым в $\mathscr{F}(\mathbb{Z}, X)$ будет подпространство $\operatorname{Im} \mathscr{D}$. Лемма доказана.

Лемма 5.8. Если $\operatorname{Im} \mathscr{D}$ - замкнутое дополняемое в $\mathscr{F}(\mathbb{Z}, X)$ подпространство и

$$
\mathscr{F}(\mathbb{Z}, X)=\operatorname{Im} \mathscr{D} \oplus \widetilde{\mathscr{F}},
$$

где $\widetilde{\mathscr{F}}$ - замкнутое подпространство, то подпространство $\operatorname{Im} \mathscr{N}$ из $\operatorname{Im} Q_{+}(0)$ замкнуто и дополняемо в $\operatorname{Im} Q_{+}(0)$.

ДокАЗАТЕЛЬство. Замкнутость подпространства $\operatorname{Im} \mathscr{N}$ следует из леммы 5.7. Пусть $\mathscr{P}_{-}, \mathscr{P}_{+}-$пара проекторов, осуществляющих разложение $\mathscr{F}(\mathbb{Z}, X)=\mathscr{F}_{-} \oplus \mathscr{F}_{+}$, т. е. $\operatorname{Im} \mathscr{P}_{\mp}=\mathscr{F}_{\mp}$. Тогда из леммы 5.5 и разложения (5.11) следует, что

$$
\mathscr{F}_{+}=\operatorname{Im} \mathscr{D}_{E}^{+} \oplus \mathscr{P}_{+}(\widetilde{\mathscr{F}}),
$$

где $\mathscr{P}_{+}(\widetilde{\mathscr{F}})$ и $\operatorname{Im} \mathscr{D}_{E}^{+}$будут замкнутыми подпространствами. Поскольку семейство $\mathscr{U}$ допускает экспоненциальную дихотомию на $\mathbb{Z}_{+}$, то из теоремы 1.11 следует, что $\operatorname{St}_{\text {inv }}\left(\mathscr{D}_{E}^{+}\right)=\operatorname{St}_{\text {reg }}\left(E, \operatorname{Im} P_{+}(0)\right)$. Поэтому из (5.12) и свойства 6) определения 1.4 следует, что $E+\operatorname{Im} P_{+}(0)$ - замкнутое (замкнутость следует из леммы 5.6) дополняемое в $X$ подпространство. Теперь из представления (5.10) подпространства $E+\operatorname{Im} P_{+}(0)$ следует дополняемость $\operatorname{Im} \mathscr{N}$ в $\operatorname{Im} Q_{+}(0)$. Лемма доказана.

Лемма 5.9. Если $\operatorname{Im} \mathscr{N}$ - замкнутое дополняемое в $\operatorname{Im} Q_{+}(0)$ подпространство, то подпространство $\operatorname{Im} \mathscr{D}$ замкнуто и дополняемо в $\mathscr{F}(\mathbb{Z}, X)$.

ДокАЗАТЕЛЬство. Из леммы 5.7 следует, что подпространство $\operatorname{Im} \mathscr{D}_{E}^{+}$замкнуто, и, следовательно, $E$ - замкнутое подпространство из $X$. Дополняемость подпространства $\operatorname{Im} \mathscr{N}$ в $\operatorname{Im} Q_{+}(0)$ в силу равенств (5.10) ведет к замкнутости и дополняемости в $X$ подпространства $E+\operatorname{Im} P_{+}(0)$. Следовательно, из теоремы 1.11 следует дополняемость в $\mathscr{F}_{+}$подпространства $\operatorname{Im} \mathscr{D}_{E}^{+}$. Теперь из леммы 5.5 следует, что и подпространство $\operatorname{Im} \mathscr{D}$ дополняемо в $\mathscr{F}(\mathbb{Z}, X)$. Лемма доказана. 
ДОКАЗАТЕЛЬСТво ТЕОРЕмы 1.12. Совпадение свойств 1)-3) из определения 1.1 о ядрах операторов $\mathscr{D}$ и $\mathscr{N}_{b, a}$ следует из теоремы 5.1 и лемм $5.1,5.4$. Из лемм 5.7, 5.8, 5.9 следует совпадение остальных свойств из определения 1.1 для рассматриваемых операторов $\mathscr{D}$ и $\mathscr{N}_{b, a}$. Теорема доказана.

Теорема 5.4. Пусть для семейства эволюиионных операторов $\mathscr{U}_{d}: \Delta_{\mathbb{Z}} \rightarrow$ End $X$ выполнено предположение 1.2. Тогда

$$
\operatorname{St}_{\text {inv }}(\mathscr{D})=\operatorname{St}_{\text {reg }}\left(\operatorname{Im} Q_{-}(0), \operatorname{Im} P_{+}(0)\right) .
$$

Оператор $\mathscr{D}$ фредгольмов (полуфредголъмов) тогда и только тогда, когда подпространства $\operatorname{Im} Q_{-}(0), \operatorname{Im} P_{+}(0)$ образуют фредгольмову (полуфредголвмову) пару. Если оператор $\mathscr{D}$ фредгольмов (полуфредгольмов), то

$$
\begin{aligned}
\operatorname{dim} \operatorname{Ker} \mathscr{D} & =\operatorname{dim}\left(\operatorname{Im} Q_{-}(0) \cap \operatorname{Im} P_{+}(0)\right), \\
\operatorname{codim} \operatorname{Im} \mathscr{L} & =\operatorname{codim}\left(\operatorname{Im} Q_{-}(0)+\operatorname{Im} P_{+}(0)\right), \\
\text { ind } \mathscr{L} & =\operatorname{ind}\left(\operatorname{Im} Q_{-}(0), \operatorname{Im} P_{+}(0)\right) .
\end{aligned}
$$

ДокАЗАТЕЛЬСтво. В данном случае $a=b=0$, и поэтому узловой оператор $\mathscr{N}$ будет иметь вид

$$
\mathscr{N}: \operatorname{Im} Q_{-}(0) \rightarrow \operatorname{Im} Q_{+}(0), \quad \mathscr{N} x=Q_{+}(0) x, \quad x \in \operatorname{Im} Q_{-}(0) .
$$

Все утверждения этого раздела, связанные со свойствами ядер и образов $\mathscr{D}$ и $\mathscr{N}$, остаются в силе. Поэтому имеет место равенство

$$
\operatorname{St}_{\text {inv }}(\mathscr{D})=\operatorname{St}_{\text {inv }}(\mathscr{N})
$$

Непосредственно из вида оператора $\mathscr{N}$ и определений $1.1,1.4$ следует, что $\operatorname{St}_{\text {inv }}(\mathscr{N})=\operatorname{St}_{\text {reg }}\left(\operatorname{Im} Q_{-}(0), \operatorname{Im} P_{+}(0)\right)$. Теорема доказана.

\section{6. Критерии почти периодичности решений дифференциальных уравнений}

При изучении дифференциального уравнения (1.1) с постоянным операторным коэффициентом $A(t) \equiv A: D(A) \subset X \rightarrow X$, являющимся генератором полугруппы $U: \mathbb{R}_{+} \rightarrow \operatorname{End} X$ класса $C_{0}$ [11], [12], [45], [46], особое значение приобретают методы гармонического анализа.

Приведем несколько используемых далее определений.

Пусть $T: \mathbb{R} \rightarrow$ End $\mathscr{X}$ ( $\mathscr{X}-$ комплексное банахово пространство) - сильно непрерывное изометрическое представление. Далее банахово пространство $L^{1}(\mathbb{R})=L^{1}(\mathbb{R}, \mathbb{C})$ рассматривается в качестве банаховой алгебры со сверткой функций в качестве умножения. Формула

$$
f x=\int_{\mathbb{R}} f(\tau) T(-\tau) x d \tau, \quad f \in L^{1}(\mathbb{R}), \quad x \in X,
$$

наделяет банахово пространство $\mathscr{X}$ структурой банахова $L^{1}(\mathbb{R})$-модуля (см. [46]-[48]). Далее через $\widehat{f}: \mathbb{R} \rightarrow \mathbb{C}$ обозначается преобразование Фурье функции $f \in L^{1}(\mathbb{R})$. 
ОПРЕДЕЛЕниЕ 6.1. Спектром Бёрлинга вектора $x$ из $\mathscr{X}$ называется множество чисел $\Lambda(x)$ из $\mathbb{R}$ вида $\Lambda(x)=\left\{\lambda_{0} \in \mathbb{R} \mid f x \neq 0\right.$ для любой функции $f \in L^{1}(\mathbb{R})$, для которой $\left.\widehat{f}\left(\lambda_{0}\right) \neq 0\right\}$.

Используемые далее свойства спектра Бёрлинга векторов из $\mathscr{X}$ можно найти в [46], [47].

ОПредЕЛЕНиЕ 6.2. Пусть $\lambda_{0} \in \mathbb{R}$. Ограниченная направленность функций $\left(f_{\alpha}\right)$ из $L^{1}(\mathbb{R})$ ( $\alpha$ пробегает некоторое направленное множество $\Omega$ ) называется $\lambda_{0}$-направленностъю, если выполнены условия:

1) $\widehat{f_{\alpha}}(0)=1$ для любого $\alpha \in \Omega$;

2) $\lim f_{\alpha} * f=0$ для любой $f \in L^{1}(\mathbb{R})$ с $\widehat{f}\left(\lambda_{0}\right)=0$.

Примерами $\lambda_{0}$-направленностей в алгебре $L^{1}(\mathbb{R})$ являются направленности: $g_{\alpha}(t)=f_{\alpha}(t) \exp \left(i \lambda_{0} t\right), \psi_{\alpha}(t)=\varphi_{\alpha}(t) \exp \left(i \lambda_{0} t\right), \alpha>0$, где 0-направленности $\left(f_{\alpha}\right)$ и $\left(\varphi_{\alpha}\right)$ определены формулами

$$
\begin{aligned}
f_{\alpha}(t) & = \begin{cases}\alpha \exp (-\alpha t), & t \geqslant 0, \\
0, & t<0,\end{cases} \\
\varphi_{\alpha}(t) & = \begin{cases}(2 \alpha)^{-1}, & t \in[-\alpha, \alpha], \\
0, & t \notin[-\alpha, \alpha],\end{cases}
\end{aligned}
$$

При этом множество $\Omega=(0, \infty)$ для $\left(f_{\alpha}\right)$ направлено по убыванию чисел, а для $\left(\varphi_{\alpha}\right)$ - по возрастанию.

ОПРЕДЕЛЕНИЕ 6.3. Число $\lambda_{0}$ из спектра Бёрлинга $\Lambda\left(x_{0}\right)$ вектора $x_{0} \in \mathscr{X}$ называется эргодической точкой спектра вектора $x_{0}$, если для некоторой $\lambda_{0}$-направленности $\left(f_{\alpha}\right)$ из алгебры $L^{1}(\mathbb{R})$ (и, следовательно, для любой $\lambda_{0}$-направленности) существует $\lim f_{\alpha} x$.

Для множества эргодических точек вектора $x_{0} \in \mathscr{X}$ используют обозначение $\Lambda_{\text {erg }}\left(x_{0}\right)$. Множество

$$
\begin{aligned}
& \Lambda_{B}(x)=\left\{\lambda_{0} \in \Lambda_{\mathrm{erg}}(x) \mid \lim f_{\alpha} x\right.=x_{0} \neq 0 \\
&\text { для некоторой } \left.\lambda_{0} \text {-направленности }\left(f_{\alpha}\right)\right\}
\end{aligned}
$$

назовем спектром Бора вектора $x \in \mathscr{X}$. Поскольку этот предел не зависит от выбора направленности $\left(f_{\alpha}\right)$ из алгебры $L^{1}(\mathbb{R})($ см. [47; гл. II]), то

$$
\begin{aligned}
\lim _{\alpha \rightarrow \infty} 2 \alpha^{-1} \int_{-\alpha}^{\alpha} T(s) e^{-i \lambda s} x d s & =\lim _{\varepsilon \rightarrow 0} \varepsilon \int_{0}^{\varepsilon^{-1}} e^{-\varepsilon \tau} T(\tau) e^{-i \lambda \tau} x d \tau \\
& =\lim _{0<\varepsilon \rightarrow 0} \varepsilon R(\varepsilon+i \lambda, i A) x,
\end{aligned}
$$

где $i A: D(A) \subset X \rightarrow X-$ генератор рассматриваемой группы операторов $T: \mathbb{R} \rightarrow$ End $\mathscr{X}$. Отметим, что $\sigma(A) \subset \mathbb{R}$, а в равенствах (6.3) используются направленности, определенные формулами (6.1), (6.2).

ОПредЕЛЕНИЕ 6.4. Вектор $x \in \mathscr{X}$ называется почти периодическим вектором, если его орбита $\{T(\tau) x, \tau \in \mathbb{R}\}$ предкомпактна в $\mathscr{X}$. 
Множество почти периодических векторов из $\mathscr{X}$ образует замкнутое подпространство в $\mathscr{X}$, обозначаемое далее через $A P(\mathscr{X})=A P(\mathscr{X}, T)$. Приводимые понятия и результаты о почти периодических векторах содержатся в работах [47], [48].

Если $x \in A P(\mathscr{X})$, то через $x \sim \sum_{\lambda \in \Lambda_{B}(x)} x_{\lambda}$ обозначается ряд Фуръе вектора $x$. Вектор $x_{\lambda}, \lambda \in \Lambda_{B}(x)$, определяется формулой $x_{\lambda}=\lim f_{\alpha} x$ для некоторой $\lambda$-направленности $\left(f_{\alpha}\right)$. Отметим, что множество $\Lambda_{B}(x)$ не более чем счетно и

$$
T(\tau) x_{\lambda}=\exp (i \lambda \tau) x_{\lambda}, \quad \tau \in \mathbb{R}, \quad \lambda \in \Lambda_{B}(x) .
$$

Если $\mathscr{X}_{0}$ - замкнутый подмодуль из $\mathscr{X}$, инвариантный относительно операторов $T(t), t \in \mathbb{R}$, то факторпространство $\mathscr{X} / \mathscr{X}_{0}$ также наделяется структурой банахова модуля по представлению $\widetilde{T}(t) \widetilde{x}=\widetilde{T(t)} x=T(t) x+\mathscr{X}_{0}$, т. е. $f \widetilde{x}=\widetilde{f x}$, для любого $x \in \mathscr{X}$. Спектр Бёрлинга $\Lambda(\widetilde{x})$ класса эквивалентности $\widetilde{x}=x+\mathscr{X}_{0}$, содержащего вектор $x$, обозначим символом $\Lambda\left(x, \mathscr{X}_{0}\right)$. Если $\mathscr{X}_{0}=A P(\mathscr{X})$, то множество $\Lambda\left(x, \mathscr{X}_{0}\right)$ назовем множеством не почти периодичности вектора $x \in \mathscr{X}$.

Теорема 6.1 [48]. Пусть для вектора $x \in \mathscr{X}$ множество $\Lambda(x, A P(\mathscr{X}))$ не более чем счетно. Для его почти периодичности необходимо и достаточно, чтобы любая предельная точка множества $\Lambda(x, A P(\mathscr{X}))$ была эргодической для вектора $x$. В частности, если множество $\Lambda(x, A P(\mathscr{X}))$ не имеет конечных предельных точек, то $x \in A P(\mathscr{X})$.

Пусть теперь $\mathscr{X}=C_{b, u}(\mathbb{R}, X) / C_{0}(\mathbb{R}, X)$. В $\mathscr{X}$ действует сильно непрерывная изометрическая группа операторов

$$
T(t) \widetilde{x}=\widetilde{S(t) x}=S(t) x+C_{0}(\mathbb{R}, X), \quad t \in \mathbb{R} .
$$

Следовательно, $\mathscr{X}$ по представлению $T$ наделяется структурой банахова $L^{1}(\mathbb{R})$ модуля.

Эта модульная структура определяется формулой

$$
\varphi \widetilde{x}=\widetilde{\varphi * x}, \quad(\varphi * x)(t)=\int_{\mathbb{R}} \varphi(t-s) x(s) d s, \quad t \in \mathbb{R},
$$

где $\varphi \in L^{1}(\mathbb{R}), x \in C_{b, u}, \widetilde{x}=x+C_{0}(\mathbb{R}, \mathbb{X})$.

ЗАмечАнИЕ 6.1. Каждую функцию $x_{+} \in C_{b, u}\left(\mathbb{R}_{+}, X\right)$ продолжим до функции $x \in C_{b, u}(\mathbb{R}, X)$ так, чтобы $\lim _{t \rightarrow-\infty} x(t)=0$. Тогда класс (эквивалентности) $\widetilde{x} \in \mathscr{X}$ не зависит от способа такого продолжения на $\mathbb{R}$ и, следовательно, банахово пространство $C_{b, u}\left(\mathbb{R}_{+}, X\right) / C_{0}\left(\mathbb{R}_{+}, X\right)$ изометрически вкладывается в $\mathscr{X}=C_{b, u}(\mathbb{R}, X) / C_{0}(\mathbb{R}, X)$ в качестве замкнутого подмодуля, которое обозначим через $\mathscr{X}_{+}$. При этом группа операторов $T(t), t \in \mathbb{R}$, корректно определена на $\mathscr{X}_{+}$.

Далее через $\mathbb{J}$ обозначается один из промежутков $\mathbb{R}_{+}, \mathbb{R}$. 
ОПРЕДЕЛЕНИЕ 6.5. Функция $x \in C_{b, u}(\mathbb{J}, X)$ называется почти периодической на бесконечности (на $\infty)$, если $x \in A P(\mathscr{X})\left(x \in A P\left(\mathscr{X}_{+}\right)\right.$, если $x \in$ $\left.C_{b, u}\left(\mathbb{R}_{+}, X\right)\right)$. Множество функций из $C_{b, u}(\mathbb{J}, X)$, почти периодических на бесконечности, обозначим символом $A P_{\infty}(\mathbb{J}, X)$.

ОПРЕДЕЛЕНИЕ 6.6. Функция $x \in C_{b, u}(\mathbb{J}, X)$ называется медленно меняющейся на бесконечности (на $\infty)$, если $S(t) x-x \in C_{0}(\mathbb{J}, X)$ для любого $t \in \mathbb{J}$.

Множество медленно меняющихся на $\infty$ функций из $C_{b, u}(\mathbb{J}, X)$, обозначаемое через $C_{s \ell, \infty}(\mathbb{J}, X)$, образует замкнутое подпространство. Примером функции из $C_{s \ell, \infty}(\mathbb{R}, \mathbb{C})$ является функция $x(t)=\sin \ln \left(1+t^{2}\right), t \in \mathbb{R}$.

Из определения спектра Бёрлинга векторов и определения 6.6 вытекает следующая лемма.

Лемма 6.1. Функция $x \in C_{b, u}(\mathbb{J}, X)$ медленно меняется на бесконечности тогда и только тогда, когда выполнено одно из следующих двух эквивалентнъхх условий: 1) $\Lambda(\widetilde{x})=\{0\} ; 2) f \widetilde{x}=\widehat{f}(0) \widetilde{x}$ для любой функции $f \in L^{1}(\mathbb{R})$, где

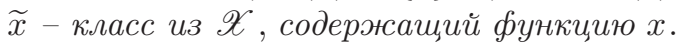

Отметим, что подпространство $C_{s \ell, \infty}\left(\mathbb{R}_{+}, X\right)$ совпадает с множеством функций из $C_{b, u}\left(\mathbb{R}_{+}, X\right)$, стационарных на бесконечности (определение см. [7; гл. III, $\S 6])$.

ОПРЕДЕЛЕНИЕ 6.7 . Пусть $x \in A P_{\infty}(\mathbb{J}, X)$ и

$$
\widetilde{x} \sim \sum_{n \geqslant 1} y_{n}, \quad \Lambda_{B}(\widetilde{x})=\left\{\lambda_{1}, \lambda_{2}, \ldots\right\}, \quad \Lambda\left(y_{n}\right)=\left\{\lambda_{n}\right\}
$$

- ряд Фурье класса $\widetilde{x} \in A P(\mathscr{X})$. Ряд

$$
x \sim \sum_{n \geqslant 1} x_{n}
$$

где $x_{n}$ - некоторый представитель класса $y_{n} \in A P(\mathscr{X})$, называется рядом $\Phi y$ ръе функщии $x$.

Отметим неединственность ряда Фурье (6.6) любой функции $x \in A P_{\infty}(\mathbb{J}, X)$. Из равенств (6.5) и леммы 6.1 следует, что каждая из функций $x_{n}, n \geqslant 1$, представима в виде $x_{n}(t)=x_{n}^{0}(t) \exp \left(i \lambda_{n} t\right), t \in \mathbb{R}$, где $x_{n}^{0} \in C_{s \ell, \infty}(\mathbb{J}, X)$.

Следовательно, имеет место (используется теорема 3.67 из [47]) следующая теорема.

ТЕОРема 6.2. Для того чтобы функиця $C_{b, u}(\mathbb{J}, X)$ была почти периодической на $\infty$, необходимо и достаточно, чтобы для любого $\epsilon>0$ существовали функции $x_{1}, \ldots, x_{n} \in C_{b, u}(\mathbb{J}, X)$, представимые в виде $x_{k}(t)=x_{k}^{0}(t) \exp \left(i \lambda_{k} t\right)$, $t \in \mathbb{R}, \lambda_{k} \in \mathbb{R}, x_{k}^{0} \in C_{s \ell, \infty}(\mathbb{R}, X)$, такие, что

$$
\sup _{t \in \mathbb{J}}\left\|x(t)-\sum_{k=1}^{n} x_{k}^{0}(t) \exp \left(i \lambda_{k} t\right)\right\|<\epsilon .
$$


Перейдем к рассмотрению дифференциального уравнения (1.1) с функцией $f \in A P_{\infty}(\mathbb{J}, X)$ с постоянным операторным коэффициентом $A(t) \equiv A, t \in \mathbb{J}$, являющимся генератором полугруппы $U: \mathbb{R}_{+} \rightarrow \operatorname{End} X$ класса $C_{0}$. Под ограниченным (слабым) решением этого уравнения понимается функция $x \in C_{b}(\mathbb{J}, X)$, для которой верны равенства

$$
x(t)=U(t-s) x(s)-\int_{s}^{t} U(t-\tau) f(\tau) d \tau, \quad t, s \in \mathbb{J}, \quad s \leqslant t,
$$

т. е. $x \in D(\mathscr{L}), \mathscr{L}=-d / d t+A$, если $\mathbb{J}=\mathbb{R}$, и $x \in D\left(\mathscr{L}_{X}^{+}\right)$, если $\mathbb{J}=\mathbb{R}_{+}$. Из (6.7) следует, что $x \in C_{b, u}(\mathbb{J}, X)$. Следовательно, подпространство $C_{b, u}(\mathbb{J}, X)$ является инвариантным для операторов $\mathscr{L}($ если $\mathbb{J}=\mathbb{R})$ и $\mathscr{L}_{X}^{+}\left(\right.$если $\left.\mathbb{J}=\mathbb{R}_{+}\right)$. Далее рассматриваются их сужения на $C_{b, u}(\mathbb{J}, X)$, которые будут обозначаться теми же символами $\mathscr{L}, \mathscr{L}_{X}$.

ТЕОРема 6.3. Для любого решения $x_{0} \in C_{b, u}(\mathbb{J}, X)$ дифференииального уравнения (1.1) с $f \in A P_{\infty}(\mathbb{J}, X)$ имеет место включение

$$
\Lambda\left(\widetilde{x}_{0}, A P_{\infty}(\mathbb{J}, X)\right) \subset \sigma(A) \cap i \mathbb{R} .
$$

Функиия $x_{0}$ почти периодична на бесконечности, если множество $\sigma(A) \cap i \mathbb{R}$ не более чем счетно и каждая предельная точка множества $\Lambda\left(\widetilde{x}_{0}, A P_{\infty}(\mathbb{J}, X)\right)$ является эргодической для класса $x_{0}+C_{0}(\mathbb{J}, X) . B$ частности, $x_{0} \in A P_{\infty}(\mathbb{J}, X)$, если множество $\sigma(A) \cap i \mathbb{R}$ не имеет конечных предельных точек.

ДоказАТЕльство. Пусть вначале $\mathbb{J}=\mathbb{R}$. Число $\lambda_{0} \in \mathbb{R}$ выберем так, чтобы $\lambda_{0}>\alpha$, где число $\alpha$ взято из определения семейства эволюционных операторов (см. раздел 1$)$. Тогда оператор $\mathscr{L}-\lambda_{0} I$ непрерывно обратим и обратный $B=$ $\left(\mathscr{L}-\lambda_{0} I\right)^{-1} \in$ End $C_{b, u}(\mathbb{R}, X)$ представим в виде $B x=G_{0} * x, x \in C_{b, u}(\mathbb{R}, X)$, где $G_{0}(\tau)=U(\tau) \exp \left(-\lambda_{0} \tau\right)$ при $\tau \geqslant 0$ и $G_{0}(\tau)=0$ при $\tau<0$. Следовательно, оператор $B$ перестановочен с операторами свертки, т. е.

$$
B(\varphi * x)=\varphi * B x, \quad \varphi \in L^{1}(\mathbb{R}), \quad x \in C_{b, u}(\mathbb{R}, X) .
$$

Поэтому тем же свойством обладает оператор $\mathscr{L}$. В частности, $\varphi * x_{0} \in D(\mathscr{L})$ и

$$
\mathscr{L}\left(\varphi * x_{0}\right)=\varphi * f \in A P_{\infty}(\mathbb{R}, X)
$$

для любой $\varphi \in L^{1}(\mathbb{R})$. Пусть $i \mu \bar{\epsilon} \sigma(A) \cap i \mathbb{R}$, где $\mu_{0} \in \mathbb{R}$. Тогда резольвента оператора $A$ определена в некоторой окрестности $i V_{0} \subset i \mathbb{R}$ точки $i \mu_{0}$. Пусть $\left[\mu_{0}-\delta, \mu_{0}+\delta_{0}\right] \subset V_{0}$, где $\delta_{0}>0$. Рассмотрим бесконечно дифференцируемую функцию $\widehat{\varphi}_{0}: \mathbb{R} \rightarrow \mathbb{C}$, для которой $\widehat{\varphi}_{0}\left(\mu_{0}\right) \neq 0$ и $\operatorname{supp} \widehat{\varphi}_{0} \subset\left[\mu_{0}-\delta, \mu_{0}+\delta\right]$. Тогда она является преобразованием Фурье некоторой функции $\varphi_{0} \in L^{1}(\mathbb{R})$, а функция

$$
\widehat{F}(\lambda)= \begin{cases}\widehat{\varphi}_{0}(\lambda) R(i \lambda, A), & \lambda \in\left[\mu_{0}-\delta, \mu_{0}+\delta\right], \\ 0, & \lambda \bar{\epsilon}\left[\mu_{0}-\delta, \mu_{0}+\delta\right],\end{cases}
$$

есть преобразование некоторой суммируемой функции $F: \mathbb{R} \rightarrow \operatorname{End} X$. Тогда из (6.9) получаем

$$
F * \mathscr{L}\left(\varphi * x_{0}\right)=\varphi_{0} * x_{0}=F * \varphi * f \in A P_{\infty}(\mathbb{R}, X) .
$$


Следовательно, из определения множества не почти периодичности следует, что $\mu_{0} \bar{\in} \Lambda\left(\widetilde{x}_{0}, A P_{\infty}(\mathbb{R}, X)\right)$.

Итак, включение (6.8) доказано для функций, определенных на $\mathbb{R}$.

Пусть теперь $\mathbb{J}=\mathbb{R}_{+}$, и тогда $\mathscr{L}_{X}^{+} x_{0}=f$. Рассмотрим непрерывно дифференцируемую функцию $\varphi: \mathbb{R} \rightarrow \mathbb{R}$ со свойствами $\operatorname{supp} \varphi \subset[1, \infty), \varphi \equiv 1$ на $[2, \infty)$. Далее символ $\varphi x_{0}$ обозначает функцию, равную нулю на $\mathbb{R}_{-}$и являющуюся произведением $\varphi$ и $x_{0}$ на $\mathbb{R}_{+}$. Тогда $\varphi x_{0} \in D(\mathscr{L})$ и $\mathscr{L}\left(\varphi x_{0}\right)=\dot{\varphi} x_{0}+\varphi f$, где $\varphi f$ считается равной нулю на $\mathbb{R}_{-}$. Таким образом, случай $\mathbb{J}=\mathbb{R}_{+}$сведен к рассмотренному. Теорема доказана.

Tеорема 6.4. Пусть полугруппа $U: \mathbb{R}_{+} \rightarrow$ End $X$ равномерно ограничена, множество $\sigma(A) \cap i \mathbb{R}$ не более чем счетно и $x_{0} \in X$.Функиия $x(t)=U(t) x_{0}$, $x: \mathbb{R}_{+} \rightarrow X$ почти периодична на бесконечности, если в каждой предельной точке $i \lambda_{0}$ множества $\sigma(A) \cap i \mathbb{R}$ существует $\lim _{0<\epsilon \rightarrow 0} \epsilon R\left(\epsilon+i \lambda_{0}, A\right) x_{0}$.

ДокАЗАТЕЛЬство следует из теоремы 6.3 и теоремы 6.2 , где в качестве $\lambda_{0}$-направленности выбирается $\lambda_{0}$-направленность $g_{\alpha}(t)=f_{\alpha}(t) \exp \left(i \lambda_{0} t\right), \alpha>0$, где $\left(f_{\alpha}\right)$ - направленность вида (6.1).

Tеорема 6.5. Пусть полугруппа $U$ равномерно ограничена и множество $\sigma(A) \cap i \mathbb{R}$ не более чем счетно. Для того чтобы полугруппа $U$ была сильно устойчивой, необходимо и достаточно, чтобы выполнялось любое из следующих условий:

1) $\lim _{0<\epsilon \rightarrow 0} \epsilon R\left(i \lambda_{0}+\epsilon, A\right) x_{0}=0$ для любого вектора $x_{0} \in X$ и для любого $i \lambda_{0} \in \sigma(A) \cap i \mathbb{R}$

2) сопряженный оператор $A^{*}$ не имеет собственных значений на $i \mathbb{R}$.

ДокАзАтЕльство. Утверждение теоремы следует из эргодической теоремы, устанавливающей эквивалентность условий 1), 2) (см., например, теорему 2.2.6 из [47]). Теорема доказана.

Утверждение этой теоремы получено в статьях [49], [50].

\section{7. Комментарии к основным понятиям и некоторым результатам из разделов 2-6}

Вначале отметим некоторые дополнительные результаты. В статьях [51]-[58] изучаются разностные и дифференциальные включения, а также получено описание спектра дифференциальных и разностных операторов в весовых пространствах (см. [54]-[58]). Оценки нормы оператора, обратного к оператору $\mathscr{L}=\mathscr{L}_{u}$, и приложения к доказательству теоремы Герхарда-Прюсса рассматривались в [59]-[72]. Метод подобных операторов в вопросах расщепления дифференциальных операторов применялся в [73]-[80].

7.1. О выборе пространств и терминологии. Пространства $L^{p, q}=$ $L^{p, q}(\mathbb{R}, \mathbb{C}), 1 \leqslant p, q \leqslant \infty$, были введены в рассмотрение в статье [81] и для них применялся термин "амальгама $L^{q}$ и $\ell^{p}$ " (использовалось обозначение $\left(L^{q}, \ell^{p}\right)$ ). Идея введения пространств принадлежит Н. Винеру в следующих случаях: пространства $L^{2,1}, L^{\infty, 2}$ были определены в статье [82], а пространства $L^{1, \infty}$, 
$L^{\infty, 1}$ - в статье [83]. По этой причине часто говорят о винеровской амальгаме. Пространство $S^{p}(\mathbb{R}, \mathbb{C}), p \in[1, \infty)$, изоморфное пространству $L^{\infty, p}(\mathbb{R}, \mathbb{C})$, использовалось В. Д. Степановым [84] для определения пространства почти периодических функций $A P\left(S^{p}\right)$. В статье [85] содержится ряд интересных результатов о гармоническом анализе в пространствах $L^{p, q}$, изучаются сопряженные к ним пространства.

Если $X$ - гильбертово пространство, то важным является использование гильбертова пространства $L^{2}(\mathbb{R}, X)=L^{2}$. Так, для оператора $\mathscr{L}=-d / d t+$ $A: D(\mathscr{L}) \subset L^{2} \rightarrow L^{2}$ в теореме 10.2 из [59] приведено точное значение нормы оператора $\mathscr{L}^{-1}$, что позволило в $[59 ; \S 10]$ получить конкретные оценки величин $\left\|\mathscr{L}^{-1}\right\|_{p}$ во всех пространствах $L^{p}(\mathbb{R}, X), p \in[1, \infty]$, и $S^{p}(\mathbb{R}, X), p \in[1, \infty]$.

Первый результат об эквивалентности свойства непрерывной обратимости обыкновенного дифференциального оператора $\mathscr{L}=-d / d t+A(t): D(\mathscr{L}) \subset$ $L^{2}\left(\mathbb{R}, \mathbb{C}^{n}\right) \rightarrow L^{2}\left(\mathbb{R}, \mathbb{C}^{n}\right)$ с $A \in C_{b}\left(\mathbb{R}\right.$, End $\left.\mathbb{C}^{n}\right)$ свойству экспоненциальной дихотомии семейства эволюционных операторов $\mathscr{U}: \Delta_{\mathbb{R}} \rightarrow$ End $\mathbb{C}^{n}$, построенных по $A$ (по дифференциальному уравнению (1.2)), был получен в статье [26] (см. также [86]).

В статье [87] дифференциальное уравнение (1.1) изучалось в общих функциональных пространствах, близких к однородному пространству $\mathscr{F}\left(\mathbb{R}_{+}, X\right)$. Если $X$ - нерефлексивное банахово пространство, то пространство $L^{p}(\mathbb{R}, X)^{*}$, $p \in[1, \infty)$, не изоморфно пространству $L^{q}\left(\mathbb{R}, X^{*}\right)$, где $q^{-1}+p^{-1}=1$ (см. [88]). Следовательно, возникает проблема использования сопряженных операторов для изучения рассматриваемых дифференциальных операторов.

Использование разностных операторов и отношений позволило применить технику сопряженных операторов и отношений. В монографии [10] для изучения дифференциального оператора использовалось семейство разностных операторов. В статье [89] для изучения свойства корректности оператора $\mathscr{L}=$ $\mathscr{L}_{u}: D(\mathscr{L}) \subset \mathscr{F}(\mathbb{R}, X) \rightarrow \mathscr{F}(\mathbb{R}, X) \in\left\{C_{b}(\mathbb{R}, X), S^{p}(\mathbb{R}, X)\right\}$ стал применяться (один) разностный оператор $\mathscr{D} \in \operatorname{End} \mathscr{F}(\mathbb{Z}, X)$. Практически во всех цитируемых здесь статьях автора, начиная с [90], [13], [14], исследование дифференциальных операторов велось с использованием соответствующих разностных операторов и разностных отношений (см. также статьи [91], [27], [29], [92], [54]-[58]). При этом пришлось использовать спектральную теорию линейных отношений. Применение разностных отношений началось в статье [19], где было введено в рассмотрение отношение $\mathscr{D}_{E}^{+} \in L R C\left(\mathscr{F}\left(\mathbb{Z}_{+}, X\right)\right)$ для изучения оператора $\mathscr{L}_{E}^{+} \in \operatorname{LRC}\left(\mathscr{F}\left(\mathbb{R}_{+}, X\right)\right)$.

Следует особо отметить, что в доказательстве теоремы 1.15 , основанном на использовании представления (8.1), существенно применяются отношение $\mathscr{D}_{E}^{+}$ для подходящего подпространства $E$ и разностный оператор $\mathscr{D}_{F,-}$, имеющий неплотную область определения для $F \neq X$.

В формулировке основных результатов данной статьи существенно использовались определения 1.1-1.5. Первое из них в очень близкой форме приводилось в статье [19] и монографиях [31; определение III.6.1], [34].

Отметим еще, что спектральная теория линейных отношений используется также в вопросах разрешимости и построений решений задачи Коши $x(0)=x_{0}$ 
для дифференциального уравнения

$$
F \dot{x}(t)=G x(t), \quad t \geqslant 0,
$$

где $F, G \in \operatorname{Hom}(\mathscr{X}, \mathscr{Y})(\mathscr{X}, \mathscr{Y}$ - банаховы пространства) с $\operatorname{Ker} F \neq\{0\}$. В статье [30] установлено, что разрешимость задачи Коши для этого уравнения эквивалентна разрешимости задачи Коши $x(0)=x_{0}$ для дифференциального включения

$$
\dot{x}(t) \in \mathscr{A} x(t), \quad t \in \mathbb{R}_{+},
$$

где $\mathscr{A}=F^{-1} G$ - линейное отношение на $\mathscr{X}$.

7.2. Комментарии к основным результатам. Большинство свойств оператора $\mathscr{L}_{E}^{+}$и отношения $\mathscr{D}_{E}^{+}$из теоремы 1.3 (из равенства (1.12)) были установлены в статье [19]. В теоремах 4.1 и 4.2 получены не доказанные в [19] свойства.

Теоремы 1.7-1.11 содержат часть центральных результатов статьи. Наиболее близкие результаты приведены в статье [19], но они были получены при дополнительном условии. Для рефлексивного пространства $X$ при условии обратимости операторов $\mathscr{U}(t, \tau), \tau<t, \tau, t \in \mathbb{R}_{+}$, утверждение теоремы 1.9 было получено в статье [27] для операторов $\mathscr{L}_{\{0\}}^{+}, \mathscr{L}_{X}^{+}$. Теоремы $1.7-1.11$ особенно важны в случаях, когда семейство эволюционных операторов (“коэффициенты" дифференциального оператора) стационарно на $+\infty$. Теоремы $1.7-1.10$ могут быть использованы при обосновании метода конечных сечений [93]-[95].

K наиболее важным результатам статьи относятся теоремы 1.12-1.16. Для доказательства теорем 1.12, 1.13 существенно привлекаются результаты, полученные для операторов $\mathscr{D}_{E}^{-}$и отношения $\mathscr{D}_{E}^{+}$.

В статье [96] рассматривалась спектральная теория дифференциальных операторов, действующих в пространствах функций на конечном промежутке.

Наконец отметим, что состояние рассматриваемой здесь теории до 1999 г. описано в монографии [18]. В ней подробно излагается история развития теории, приводятся первые результаты по теории дифференциальных операторов, основанные на использовании разностных операторов (излагаются результаты статьи автора [14]).

\section{8. Примеры}

Пример 8.1. Пусть функция $A: \mathbb{J} \rightarrow \operatorname{End} X$, где $\mathbb{J} \in\left\{\mathbb{R}_{+}, \mathbb{R}\right\}$, принадлежит пространству Степанова $S^{1}(\mathbb{J}$, End $X)$. Тогда (см. [7]) существует семейство эволюционных операторов $\mathscr{U}: \mathbb{J} \times \mathbb{J} \rightarrow$ End $X$, которое решает задачу Коши $(1.2),(1.5)$, и оно представимо в виде $\mathscr{U}(t, s)=U(t) U(s)^{-1}, s, t \in \mathbb{J}$, где операторная функция $U: \mathbb{J} \rightarrow$ End $X$ (функция Коши) является решением (для почти всех $t \in \mathbb{J})$ операторного дифференциального уравнения

$$
\ddot{X}+A(t) X=0, \quad t \in \mathbb{J}, \quad X(0)=I .
$$

Полученные в настоящей статье результаты содержат соответствующие результаты из монографий [6], [7], а некоторые из них являются новыми. Такими 
являются результаты, связанные с исследованием оператора $\mathscr{L}_{E}: D\left(\mathscr{L}_{E}\right) \subset$ $\mathscr{F}\left(\mathbb{R}_{+}, X\right) \rightarrow \mathscr{F}\left(\mathbb{R}_{+}, X\right)$ (в [6], [7] рассматривались операторы $\mathscr{L}_{\{0\}}$ и $\left.\mathscr{L}_{X}\right)$. Поскольку операторы $U(t), t \in \mathbb{J}$, непрерывно обратимы, то экспоненциальная дихотомия семейства $\mathscr{U}$ на одном из бесконечных промежутков $(-\infty, a]$, $[b, \infty)$ влечет экспоненциальную дихотомию семейства $\mathscr{U}$ на любом соответствующем промежутке $\left(-\infty, a^{\prime}\right], a^{\prime}>a$; $\left[b^{\prime}, \infty\right), b^{\prime}<b$. Поэтому при исследовании оператора $\mathscr{L}=-d / d t+A(t): D(\mathscr{L}) \subset \mathscr{F}\left(\mathbb{R}_{+}, X\right) \rightarrow \mathscr{F}\left(\mathbb{R}_{+}, X\right)$ при условии экспоненциальной дихотомии семейства $\mathscr{U}: \mathbb{R} \times \mathbb{R} \rightarrow$ End $X$ на промежутках $(-\infty, 0],[0, \infty)$ естественно использовать теорему 1.14 . В частности, из нее вытекает следующий часто используемый в теории динамических систем результат В.А. Плисса [97] (в его формулировке применяется используемая здесь терминология).

Tеорема 8.1. Пусть $\operatorname{dim} X<\infty$ и семейство $\mathscr{U}: \mathbb{R} \times \mathbb{R} \rightarrow$ End $X$ обладает свойством экспоненциальной дихотомии на $\mathbb{R}_{-} u \mathbb{R}_{+}$с расщепляющими парами $P_{ \pm}, Q_{ \pm}: \mathbb{R}_{ \pm} \rightarrow$ End $X$ проекторнозначных функиий, причем $\operatorname{Im} Q_{-}(0)+$ $\operatorname{Im} P_{+}(0)=X$. Тогда оператор $\mathscr{L}=-d / d t+A(t): D(\mathscr{L}) \subset C_{b}(\mathbb{R}, X) \rightarrow C_{b}(\mathbb{R}, X)$ сюоргективен.

Пример 8.2. Рассмотрим уравнение (1.1), где $X=L^{2}(\Omega, \mathbb{C})$ и $\Omega$ - ограниченная область с гладкой границей в $\mathbb{R}^{n}$. Семейство линейных дифференциальных операторов

$$
A(t): H_{0}^{m}(\Omega) \cap H^{2 m}(\Omega) \subset L_{2}(\Omega) \rightarrow L_{2}(\Omega), \quad t \in \mathbb{J} \in\left\{\mathbb{R}_{-}, \mathbb{R}_{+}\right\}
$$

(где $H_{0}^{m}(\Omega), H^{2 m}(\Omega)$ - пространства Соболева, $m \geqslant 1$, см. [5]) определено с помощью семейства дифференциальных выражений

$$
\left(\ell_{t} y\right)(n)=\sum_{|\alpha| \leqslant 2 m} a_{\alpha}(t, u)\left(D^{\alpha} y\right)(u), \quad t \geqslant 0,
$$

и задачей Дирихле на границе $\partial \Omega$ области $\Omega$. Функции $a_{\alpha}: \mathbb{R}_{+} \times \Omega \rightarrow \mathbb{C}$, где $|\alpha| \leqslant 2 m$, принадлежат пространству $C_{b}\left(\mathbb{R}_{+}, C^{k}(\Omega)\right)$ для достаточно большого $k \in \mathbb{N}$ и удовлетворяют условию Липшица как функции первой переменной. Более того, предположим, что семейство дифференциальных выражений $\ell_{t}$, $t \geqslant 0$, равномерно эллиптично.

Из сделанных предположений следует, что эллиптические операторы $A(t)$, $t \in \mathbb{J}$, являются генераторами аналитических полугрупп операторов, более того, выполнены условия теоремы Соболевского-Танабе, из которой следует, что рассматриваемая задача Коши на $\mathbb{J}$ корректно определена и существует семейство эволюционных операторов $\mathscr{U}: \Delta_{\mathbb{J}} \rightarrow$ End $L_{2}$, позволяющее решить рассматриваемую задачу Коши. Поэтому определен оператор $\mathscr{L}_{E}^{+}=-d / d t+A(t)$ в любом из однородных пространств функций $\mathscr{F}\left(\mathbb{R}_{+}, X\right)$, следовательно, к нему применимы полученные здесь результаты.

Отметим еще, что все операторы $\mathscr{U}(t, s), s<t, s, t \in \mathbb{J}$, являются компактными операторами. Предположим, что для семейства $\mathscr{U}$ выполнено предположение 1.1. Следовательно, $\operatorname{Im} Q_{-}(a), \operatorname{Im} Q_{+}(b)$ - конечномерные подпространства из $L_{2}(\Omega)$, и, значит, оператор $\mathscr{L}=-d / d t+A(t): D(\mathscr{L}) \subset \mathscr{F}(\mathbb{R}, X) \rightarrow \mathscr{F}(\mathbb{R}, X)$ фредгольмов, если $\mathbb{J}=\mathbb{R}$. 
Пример 8.3. Пусть $A: D(A) \subset X \rightarrow X$ - генератор сильно непрерывной полугруппы операторов $U: \mathbb{R}_{+} \rightarrow$ End $X$. Рассмотрим дифференциальное уравнение (1.2), где $t \in \mathbb{J} \in\left\{\mathbb{R}_{+}, \mathbb{R}\right\}$. Соответствующее ему семейство эволюционных операторов $\mathscr{U}: \Delta_{\mathbb{J}} \rightarrow$ End $X$ имеет вид

$$
\mathscr{U}(t, s)=T(t-s), \quad s \leqslant t, \quad s, t \in \mathbb{J} .
$$

Из теоремы 1.5 следует, что дифференциальный оператор

$$
\mathscr{L}=-\frac{d}{d t}-A: D(\mathscr{L}) \subset \mathscr{F}(\mathbb{R}, X) \rightarrow \mathscr{F}(\mathbb{R}, X)
$$

непрерывно обратим тогда и только тогда, когда обратим разностный оператор $\mathscr{D} \in \operatorname{End} \mathscr{F}(\mathbb{Z}, X),(\mathscr{D} x)(n)=x(n)-T(1) x(n-1), n \in \mathbb{Z}, x \in \mathscr{F}(\mathbb{Z}, X)$. Из результатов статьи [30] следует, что непрерывная обратимость оператора $\mathscr{D}$ эквивалентна следующему условию (называемому условием гиперболичности полугруппы $U)$ :

$$
\sigma(U(1)) \cap \mathbb{T}=\varnothing
$$

где $\mathbb{T}=\{\lambda \in \mathbb{C}|| \lambda \mid=1\}$ - единичная окружность. Из условия (8.1) следует, что выполнены условия

$$
\sigma(A) \cap i \mathbb{R}=\varnothing, \quad \sup _{\lambda \in \mathbb{R}}\|R(i \lambda, A)\|<\infty .
$$

Выполнение условий (8.2) не всегда влечет выполнение условия (8.1). Однако, если $X$ - гильбертово пространство, то из теоремы Герхарда-Прюсса (см. [60], [61], [59]) следует, что условия (8.1) имеют место. Из (8.1) получаем представление

$$
\sigma(U(1))=\sigma_{\text {int }} \cup \sigma_{\text {out }},
$$

где $\sigma_{\text {int }}=\{\lambda \in \sigma(U(1))|| \lambda \mid 1\}$. Пусть $P_{\text {int }}$ и $P_{\text {out }}-$ проекторы Рисса, построенные по спектральным множествам $\sigma_{\text {int }}$ и $\sigma_{\text {out }}$ соответственно. Тогда $X=X_{\text {int }} \oplus X_{\text {out }}$, где $X_{\text {int }}=\operatorname{Im} P_{\text {int }}, X_{\text {out }}=\operatorname{Im} P_{\text {out }}$. Подпространство $X_{\text {int }}$ называют устойчивым, а $X_{\text {оut }}$ - неустойчивым.

Если оператор $\mathscr{L}=-d / d t+A: D(\mathscr{L}) \subset \mathscr{F}(\mathbb{R}, X) \rightarrow \mathscr{F}(\mathbb{R}, X)$ фредгольмов, то в силу теоремы 1.16 семейство $\mathscr{U}$ допускает экспоненциальную дихотомию на $\mathbb{R}_{+}$. Тогда из [19; теорема 10.1$]$ следует, что полугруппа операторов $U$ гиперболична, т. е. выполнено условие (8.1), что влечет непрерывную обратимость оператора $\mathscr{L}$.

ПримеР 8.4 (системы дифференциальных уравнений, корректные по Петровскому). Пусть $p(\xi)=\left(p_{k j}\right)_{k, j=1}^{m}, \xi \in \mathbb{R}^{n}, n \in \mathbb{N},-$ матрица, элементами которой служат полиномы $p_{k j}: \mathbb{R}^{n} \rightarrow \mathbb{C}, p_{k j}(\xi)=\sum_{|\alpha| \leqslant N_{k j}} a_{\alpha} \xi^{\alpha}$. Здесь $\alpha \in \mathbb{N}_{+}^{n}$, $a_{\alpha} \in \mathbb{C}$, они зависят от $k$ и $j$. В гильбертовом пространстве оператор $A=p(i \partial)$, $\partial=\left(\partial_{1}, \ldots, \partial_{n}\right), i^{2}=-1$, определяется с помощью преобразования Фурье $\mathscr{F}$ формулой $A=\mathscr{F}^{-1} p(\cdot) \mathscr{F}$, он является оператором с матричными коэффициентами, имеющим символ $p$. Говорят, что $A$ корректен по Петровскому, если для некоторого $\omega \in \mathbb{R}$ спектр $\sigma(p(\xi))$ матрицы $p(\xi)$ удовлетворяет включению 
$\sigma(p(\xi)) \subset\{\lambda \in \mathbb{C} \mid \operatorname{Re} \lambda \leqslant \omega\}$ для всех $\xi \in \mathbb{R}^{n}$. В этом случае $A$ порождает сильно непрерывную полугруппу операторов $T: \mathbb{R}_{+} \rightarrow \operatorname{End} L_{2}\left(\mathbb{R}^{n}\right)$ и имеет область определения $D(A)$, совпадающую с пространством Соболева $W_{2}^{N}\left(\mathbb{R}^{n}\right)$ порядка $N$. Эта полугруппа гиперболична (оператор $T(1)$ обладает свойством $\sigma(T(1)) \cap \mathbb{T}=\varnothing)$, если множества $\sigma(p(\xi)), \xi \in \mathbb{R}^{n}$, равномерно отделены от $i \mathbb{R}$. Тогда семейство $\mathscr{U}(t, s)=T(t-s), s \leqslant t, s, t \in \mathbb{R}_{+}$, допускает экспоненциальную дихотомию на $\mathbb{R}_{+}$. В этом случае устойчивые и неустойчивые подпространства бесконечномерны, если они ненулевые.

ПримеР 8.5. Пусть $S: L^{p}\left(\mathbb{R}_{+}, X\right) \rightarrow L^{p}\left(\mathbb{R}_{+}, X\right)=L^{p}-$ оператор ВинераХопфа,

$$
(S x)(t)=x(t)-\int_{0}^{\infty} \mathscr{K}(t-s) x(s) d s, \quad t \in \mathbb{R}_{+}, \quad x \in L^{p},
$$

ядро $\mathscr{K}: \mathbb{R} \rightarrow$ End $X$ которого суммируемо и имеет символ $W$ вида

$$
W(\lambda)=I-\int_{\mathbb{R}} e^{i \lambda t} \mathscr{K}(t) d t=I-N R(\lambda, A) M, \quad \lambda \in \mathbb{R},
$$

где $i A: D(A) \subset Y \rightarrow Y$ (здесь $Y$ - вспомогательное банахово пространство) генератор полугруппы операторов $T: \mathbb{R}_{+} \rightarrow$ End $Y$ класса $C_{0}$, для которой выполнено условие $\sigma(T(1)) \cap \mathbb{T}=\varnothing$ (и, следовательно, $\sigma(A) \cap \mathbb{R}=\varnothing)$. Линейные операторы $M: X \rightarrow Y$ и $N: Y \rightarrow X$ являются ограниченными. В частности, при $X=\mathbb{C}^{m}$ в [34; разд. XIII.4] доказано, что если символ $W$ является рациональной функций, то такое представление возможно для некоторых операторов $A \in$ End $\mathbb{C}^{n}, M: \mathbb{C}^{m} \rightarrow \mathbb{C}^{n}, N: \mathbb{C}^{n} \rightarrow \mathbb{C}^{m}$ и некоторого $n \in \mathbb{N}$. Выбор подходящего банахова пространства $X$ позволяет любую функцию $W=I-\widehat{\mathscr{K}}$, голоморфную в области $\mathbb{C}_{\alpha}=\{\lambda \in \mathbb{C}|| \operatorname{Im} \lambda \mid<\alpha\}$, представить в виде $(8.3)$ при $Y=\mathbb{C}$. Нетрудно установить (см. [34; лемма 18.5.1] для $X=\mathbb{C}^{n}$ ), что оператор $S$ представим в виде $S=I+i \widetilde{N} \mathscr{L}_{E}^{-1} \widetilde{M}$, где $\widetilde{M}: L^{p}\left(\mathbb{R}_{+}, X\right) \rightarrow L^{p}\left(\mathbb{R}_{+}, Y\right)$ и $\tilde{N}: L^{p}\left(\mathbb{R}_{+}, Y\right) \rightarrow L^{p}\left(\mathbb{R}_{+}, X\right)$ - операторы умножения на операторы $M$ и $N$ соответственно,

$$
\mathscr{L}_{E}^{+}=-\frac{d}{d t}+i A: D\left(\mathscr{L}_{E}^{+}\right) \subset L^{p}\left(\mathbb{R}_{+}, Y\right) \rightarrow L^{p}\left(\mathbb{R}_{+}, Y\right), \quad E=\operatorname{Im} P_{\text {out }},
$$

где $P_{\text {out }}$ - проектор Рисса, построенный по спектральному множеству $\sigma_{\text {out }}=$ $\{\lambda \in \sigma(T(1))|| \lambda \mid>1\}$ для оператора $T(1)$. Это представление позволяет установить, что имеет место (см. [34; теорема 18.5.3] для $X=\mathbb{C}^{n}$ ) следующая теорема.

TeOpema 8.2. Пyсmъ $\widetilde{\mathscr{L}_{E}^{+}}=\mathscr{L}_{E}^{+}-M N=-d / d t+A-M N: D\left(\mathscr{L}_{E}^{+}\right) \subset$ $L^{p}\left(\mathbb{R}_{+}, X\right) \rightarrow L^{p}\left(\mathbb{R}_{+}, X\right), p \in[1, \infty]$. Тогда

$$
\operatorname{Ker} S=N\left(\operatorname{Ker} \widetilde{\mathscr{L}_{E}^{+}}\right), \quad \operatorname{Im} S=M^{-1}\left(\operatorname{Im} \widetilde{\mathscr{L}_{E}^{+}}\right)
$$

u оператор $S$ фредгольмов, если и только если фредгольмовым является оператор $\widetilde{\mathscr{L}}_{E}^{+}$, при этом их индексъ совпадают. В частности, $S$ обратим тогда и только тогда, когда обратим оператор $\widetilde{\mathscr{L}}_{E}^{+}$, и в этом случае $S^{-1}=$ $\left(I-i \widetilde{N} \widetilde{\mathscr{L}}_{E}^{-1}\right) \widetilde{M}$. 
Возникает естественный вопрос: влечет ли свойство фредгольмовости оператора $\mathscr{L}=\mathscr{L}_{\mathscr{U}}($ оператора $\mathscr{D})$ для $\mathscr{F}=\mathscr{F}_{c}$ свойство экспоненциальной дихотомии семейства $\mathscr{U}$. Отрицательный ответ на этот вопрос дает следующий пример.

Пример 8.6. Пусть $A_{0}: \mathscr{D}\left(A_{0}\right) \subset X \rightarrow X-$ генератор полугруппы операторов $T_{0}: \mathbb{R}_{+} \rightarrow \operatorname{End} X$ класса $C_{0}$, для которой $T_{0}(1)-$ фредгольмов оператор, причем $\operatorname{Ker} T_{0}(1)^{*} \neq\{0\}$. Рассмотрим функцию $A(t)=A_{0}, t \in[0,1)$, $A(t)=(\ln 2) I, t \in[1, \infty)$, и дифференциальный оператор $\mathscr{L}_{X}^{+}=-d / d t+A(t)$ в одном из однородных пространств функций $\mathscr{F}=\mathscr{F}\left(\mathbb{R}_{+}, X\right)$ со свойством $\mathscr{F}=\mathscr{F}_{c}$. Тогда $\mathscr{U}(1,0)=T_{0}(1), \mathscr{U}(n, n-1)=2 I, n \geqslant 2$, и соответствующее разностное отношение $\mathscr{D}_{X}^{+} \in \operatorname{LRC}\left(\mathscr{F}\left(\mathbb{Z}_{+}, X\right)\right)$ определяется равенством

$$
\begin{gathered}
\mathscr{D}_{X}^{+}=\left\{(x, y) \in \mathscr{F}\left(\mathbb{Z}_{+}, X\right) \mid y(n)=x(n)-2 x(n-1), n \geqslant 2,\right. \\
\left.y(1)=x(1)-T_{0}(1) x(0)\right\} .
\end{gathered}
$$

Семейство $\mathscr{U}$ допускает тривиальную экспоненциальную дихотомию на множестве $\mathbb{N}$ при $P=0$ и $Q=I$, и поэтому имеют место представления

$$
\begin{aligned}
\operatorname{Ker} \mathscr{D}_{X}^{+} & =\left\{x \in \mathscr{F}\left(\mathbb{Z}_{+}, X\right) \mid x(0)\right. \\
\operatorname{Im} \mathscr{D}_{X}^{+}=\left\{f \in \mathscr{F}\left(\mathbb{Z}_{+}, X\right) \mid f(1)\right. & \left.\in \operatorname{Im} T_{0}(1)\right\}=\overline{\operatorname{Im} \mathscr{D}_{X}^{+}}, \\
\operatorname{Ker} \mathscr{D}_{X}^{*}=\left\{\xi \in \mathscr{F}^{\prime}\left(\mathbb{Z}_{+}, X\right) \mid \xi(0)\right. & =0, \xi(1) \in \operatorname{Ker} T_{0}(1)^{*}, \\
\xi(n) & \left.=2^{-n+1} \xi(1), n \geqslant 2\right\} \neq\{0\} .
\end{aligned}
$$

Таким образом, $\mathscr{D}_{X}^{+}$- фредгольмово отношение, а из [19; лемма 8.7] следует, что семейство $\mathscr{U}$ не допускает экспоненциальной дихотомии на $\mathbb{Z}_{+}$. Из $[19 ;$ лемма 6.2] следует, что семейство эволюционных операторов $\mathscr{U}: \Delta_{\mathbb{R}} \rightarrow \operatorname{End} X$ (мы сохраняем обозначение $\mathscr{U}$ для расширения построенного семейства) вида

$$
\mathscr{U}(t, s)= \begin{cases}e^{2(t-s)} I, & 1 \leqslant s \leqslant t<\infty \\ T_{0}(t-s), & 0 \leqslant s \leqslant t \leqslant 1 \\ I, & s \leqslant t \leqslant 0\end{cases}
$$

естественным образом продолженное на $\Delta_{\mathbb{R}}$, обладает свойством экспоненциальной дихотомии на $\mathbb{R}_{-}$и на $[1, \infty)$. Однако оно не обладает таким свойством на промежутке $[0, \infty)$. В данном случае соответствующие расщепляющие пары $P_{-}, Q_{-}: \mathbb{R}_{-} \rightarrow$ End $X, P_{+}, Q_{+}: \mathbb{R}_{+} \rightarrow$ End $X$ имеют вид: $P_{-}=I, Q_{-} \equiv 0$, $P_{+} \equiv 0, Q_{+} \equiv I$. Следовательно, узловой оператор

$$
\mathscr{N}_{1,0}: \operatorname{Im} Q_{-}(0)=\{0\} \rightarrow \operatorname{Im} Q_{+}(1)=X
$$

будет нулевым. Таким образом, построенное семейство эволюционных операторов $\mathscr{U}: \Delta_{\mathbb{R}} \rightarrow$ End $X$ соответствует дифференциальному оператору $\mathscr{L}=$ $-d / d t+A(t): D(\mathscr{L}) \subset \mathscr{F}(\mathbb{R}, X) \rightarrow \mathscr{F}(\mathbb{R}, X)$, где $A(t)=0$ при $t>1$. Поскольку узловой оператор $\mathscr{N}_{1,0}$ полуфредгольмов, то таким же (в силу теоремы 1.13 ) будет и оператор $\mathscr{L}_{\mathscr{U}}$. Оператор $\mathscr{N}_{1,0}$ инъективен, и, следовательно, инъективным будет оператор $\mathscr{L}_{\mathscr{U}}: D\left(\mathscr{L}_{\mathscr{U}}\right) \subset \mathscr{F}(\mathbb{R}, X) \rightarrow \mathscr{F}(\mathbb{R}, X)$. 
Пример 8.7. Пусть $T: \mathbb{R}_{+} \rightarrow$ End $X$ - ограниченная полугруппа класса $C_{0}$, генератор $A$ которой обладает тем свойством, что $\sigma(A) \cap i \mathbb{R}=\left\{i \lambda_{1}, \ldots, i \lambda_{n}\right\}-$ конечное множество. Из результатов раздела 6 вытекает следующая теорема.

Tеорема 8.3. Полугруппа $T$ допускает представление

$$
T(t)=\sum_{k=1}^{n} B_{k}(t) e^{i \lambda_{k} t}+B_{0}(t), \quad t \geqslant 0,
$$

где $B_{k} \in C_{b, u}\left(\mathbb{R}_{+}\right.$, End $\left.X\right)$ - медленно меняющиеся на бесконечности операторнозначнъе функции и $B_{0}: \mathbb{R}_{+} \rightarrow$ End $X$ - сильно непрерьвная функиия, обладающая свойством: $\lim _{t \rightarrow \infty} B_{0}(t) x=0$ для любого вектора $x \in X$.

Отметим, что функции $B_{k}, k=1, \ldots, n$, в представлении (8.4) могут быть выбраны так, что они допускают расширение на $\mathbb{C}$ до целой функции экспоненциального (сколь угодно малого) типа, причем $\lim _{t \rightarrow \infty}\left\|B_{k}^{\prime}(t)\right\|=0, k=1, \ldots, n$. Возможность выбора появляется ввиду леммы 6.1.

Аналог теоремы 8.3 имеет место в случае, если $\sigma(A) \cap i \mathbb{R}$ - счетное множество, не имеющее предельных точек на $i \mathbb{R}$. Следует особо отметить, что числа $i \lambda_{1}, \ldots, i \lambda_{n}$ из условия теоремы не обязаны находиться в разных компонентах связности из $\sigma(A)$.

\section{Список литературы}

[1] М. Г. Крейн, "О некоторых вопросах, связанных с кругом идей Ляпунова в теории устойчивости", УМН, 3:3(25) (1948), 166-169.

[2] М. Г. Крейн, Лекиии по теории устойчивости решений дифференциальных уравнений в банаховом пространстве, Ин-т матем. АН УССР, Киев, 1964, 186 с.

[3] Б. П. Демидович, Лекиии по математической теории устойчивости, Наука, М., 1967, 472 c.

[4] W. A. Coppel, Dichotomies in stability theory, Lecture Notes in Math., 629, Springer-Verlag, Berlin-New York, 1978, ii+98 pp.

[5] Ф. Хартман, Обыкновенные дифференииальные уравнения, Мир, М., 1970, 720 с.; пер. с англ.: Ph. Hartman, Ordinary differential equations, John Wiley \& Sons, Inc., New York-London-Sydney, 1964, xiv+612 pp.

[6] Х. Л. Массера, Х. Х. Шеффер, Линейные дифференииальные уравнения и функциональные пространства, Мир, М., 1970, 456 с.; пер. с англ.: J.L. Massera, J. J. Schäffer, Linear differential equations and function spaces, Pure Appl. Math., 21, Academic Press, New York-London, 1966, xx+404 pp.

[7] Ю. Л. Далецкий, М. Г. Крейн, Устойчивость решений дифференииальных уравнений в банаховом пространстве, Нелинейный анализ и его приложения, Наука, M., 1970, 534 с.; англ. пер.: Yu. L. Daletskii, M. G. Krein, Stability of solutions of differential equations in Banach space, Transl. Math. Monogr., 43, Amer. Math. Soc., Providence, RI, 1974, vi+386 pp.

[8] В. В. Жиков, "Некоторые вопросы допустимости и дихотомии. Принцип усреднения", Изв. АН СССР. Сер. матем., 40:6 (1976), 1380-1408; англ. пер.: V. V. Zhikov, "Some admissibility and dichotomy questions. The averaging principle", Math. USSR-Izv., 10:6 (1976), 1307-1332.

[9] Б. М. Левитан, В.В. Жиков, Почти периодические функции и дифференциальнъе уравнения, Изд-во Моск. ун-та, М., 1978, 204 с.; англ. пер.: В. M. Levitan, 
V.V. Zhikov, Almost periodic functions and differential equations, Cambridge Univ. Press, Cambridge-New York, 1982, xi+211 pp.

[10] Д. Хенри, Геометрическая теория полулинейных параболических уравнений, Мир, М., 1985, 376 с.; пер. с англ.: D. Henry, Geometric theory of semilinear parabolic equations, Lecture Notes in Math., 840, Springer-Verlag, Berlin-New York, 1981, iv $+348 \mathrm{pp}$.

[11] Э. Хилле, Р. Филлипс, Функциональный анализ и полугруппы, ИЛ, М., 1962, 829 с.; пер. с англ.: E. Hille, R. S. Phillips, Functional analysis and semi-groups, rev. ed., Amer. Math. Soc. Colloq. Publ., 31, Amer. Math. Soc., Providence, RI, 1957, xii+808 pp.

[12] K.-J. Engel, R. Nagel, One-parameter semigroups for linear evolution equations, Grad. Texts in Math., 194, Springer-Verlag, New York, 2000, xxii+586 pp.

[13] А. Г. Баскаков, "Спектральный анализ линейных дифференциальных операторов и полугруппы разностных операторов", Докл. РАН, 343:3 (1995), 295-298; англ. пер.: A. G. Baskakov, "Spectral analysis of linear differential operators and semigroups of difference operators", Dokl. Math., 52:1 (1995), 30-32.

[14] А. Г. Баскаков, "Полугруппы разностных операторов в спектральном анализе линейных дифференциальных операторов", Функи. анализ и его прил., 30:3 (1996), 1-11; англ. пер.: A. G. Baskakov, "Semigroups of difference operators in spectral analysis of linear differential operators", Funct. Anal. Appl., 30:3 (1996), 149-157.

[15] А.Г. Баскаков, “Линейные дифференциальные операторы с неограниченными операторными коэффициентами и полугруппы разностных операторов", Матем. заметки, 59:6 (1996), 811-820; англ. пер.: A. G. Baskakov, "Linear differential operators with unbounded operator coefficients and semigroups of bounded operators", Math. Notes, 59:5-6 (1996), 586-593.

[16] Yu. Latushkin, S. Montgomery-Smith, "Evolutionary semigroups and Lyapunov theorems in Banach spaces", J. Funct. Anal., 127:1 (1995), 173-197.

[17] F. Räbiger, R. Schnaubelt, "The spectral mapping theorem for evolution semigroups on spaces of vector-valued functions", Semigroup Forum, 52:2 (1996), 225-239.

[18] C. Chicone, Yu. Latushkin, Evolution semigroups in dynamical system and differential equations, Math. Surveys Monogr., 70, Amer. Math. Soc., Providence, RI, 1999, $\mathrm{x}+361 \mathrm{pp}$.

[19] А. Г. Баскаков, “Спектральный анализ дифференциальных операторов с неограниченными операторными коэффициентами, разностные отношения и полугруппы разностных отношений", Изв. РАН. Сер. матем., 73:2 (2009), 3-68; англ. пер.: A. G. Baskakov, "Spectral analysis of differential operators with unbounded operator-valued coefficients, difference relations and semigroups of difference relations", Izv. Math., 73:2 (2009), 215-278.

[20] J.S. Howland, "Stationary scattering theory for time-dependent Hamiltonians", Math. Ann., 207:4 (1974), 315-335.

[21] А.Г. Баскаков, "Об обратимости и фредгольмовости параболических дифференциальных операторов", Докл. РАН, 383:5 (2002), 583-585; англ. пер.: A. G. Baskakov, "On invertibility and the Fredholm property of parabolic differential operators", Dokl. Math., 65:2 (2002), 245-247.

[22] Э. Мухамадиев, "Условия фредгольмовости дифференциальных операторов в пространстве непрерывных и ограниченных функций”, Докл. АН Тадж. ССР, 17:4 (1974), 13-16.

[23] Э. Мухамадиев, Исследование по теории периодических и ограниченных решений дифференииальных уравнений, Автореферат дисс. ... докт. физ.-матем. наук, Л., 1979. 
[24] Ю.Я. Исаенко, "Об условиях фредгольмовости линейных дифференциальных операторов в пространстве непрерывных ограниченных функций", Приближенные методы исследования дифференииальных уравнений и их приложения, Куйбышевский гос. ун-т, Куйбышев, 1982, 78-83.

[25] K. J. Palmer, "Exponential dichotomies and transversal homoclinic points", J. Differential Equations, 55:2 (1984), 225-256.

[26] A. Ben-Artzi, I. Gohberg, "Dichotomy of systems and invertibility of linear ordinary differential operators", Time-variant systems and interpolation, Oper. Theory Adv. Appl., 56, Birkhäuser, Basel, 1992, 90-119.

[27] Yu. Latushkin, Yu. Tomilov, "Fredholm differential operators with unbounded coefficients", J. Differential Equations, 208:2 (2005), 388-429.

[28] А.Г. Баскаков, "Об дифференциальных и разностных фредгольмовых оператоpax", Докл. РАН, 416:2 (2007), 156-160; англ. пер.: A. G. Baskakov, "Fredholm differential and difference operators", Dokl. Math., 76:2 (2007), 669-672.

[29] Yu. Latushkin, A. Pogan, R. Schnaubelt, "Dichotomy and Fredholm properties of evolution equqtions", J. Operator Theory, 58:2 (2007), 387-414.

[30] А.Г. Баскаков, К. И. Чернышов, "Спектральный анализ линейных отношений и вырожденные полугруппы операторов”, Матем. сб., 193:11 (2002), 3-42; англ. пер.: A. G. Baskakov, K.I. Chernyshov, "Spectral analysis of linear relations and degenerate operator semigroups", Sb. Math., 193:11-12 (2002), 1573-1610.

[31] R. Cross, Multivalued linear operators, Monogr. Textbooks Pure Appl. Math., 213, Marcel Dekker, Inc., New York, 1998, x+335 pp.

[32] A. Favini, A. Yagi, Degenerate differential equations in Banach spaces, Monogr. Textbooks Pure Appl. Math., 215, Marcel Dekker, Inc., New York, 1999, xii+313 pp.

[33] A. E. Taylor, Introduction to functional analysis, John Wiley \& Sons, Inc., New York; Chapman \& Hall, Ltd., London, 1958, xvi+423 pp.

[34] S. Goldberg, Unbounded linear operators: Theory and applications, McGraw-Hill Book Co., New York-Toronto-London, 1966, viii+199 pp.

[35] У. Рудин, Функииональный анализ, Мир, М., 1975, 443 с.; пер. с англ.: W. Rudin, Functional analysis, McGraw-Hill Ser. Higher Math., New York-Düsseldorf-Johannesburg, 1973, xiii+397 pp.

[36] Т. Като, Теория возмущений линейнъх операторов, Мир, М., 1972, 740 с.; пер. с англ.: T. Kato, Perturbation theory for linear operators, Grundlehren Math. Wiss., 132, Springer-Verlag, New York, 1966, xix +592 pp.

[37] З. Прёсдорф, Некоторые классы сингулярных уравнений, Мир, М., 1979, 493 с.; пер. с нем.: S. Prössdorf, Einige Klassen singulärer Gleichungen, Lehrbucher Monogr. Geb. Exakten Wissensch., Math. Reihe, 46, Birkhäuser Verlag, Basel-Stuttgart, 1974, xii+353 pp.

[38] С. С. Кутателадзе, Основы функиионального анализа, 4-е изд., Изд-во Ин-та матем. СО РАН, Новосибирск, 2001, xii+354 с.; англ. пер. 2-го изд.: S. S. Kutateladze, Fundamentals of functional analysis, Kluwer Texts Math. Sci., 12, Kluwer Acad. Publ., Dordrecht, 1996, xiv+276 pp.

[39] А.Г. Баскаков, "Спектральный анализ дифференциальных операторов и полугруппы разностных операторов. I", Дифферени. уравнения, 33:10 (1997), 1299-1306; "II", 37:1 (2001), 3-11; англ. пер.: A. G. Baskakov, "Spectral analysis of linear differential operators, and semigroups of difference operators. I", Differ. Equ., 33:10 (1997), 1305-1312; "II", Differ. Equ., 37:1 (2001), 1-10.

[40] A. G. Baskakov, I. A. Krishtal, "Spectral analysis of operators with the two-point Bohr spectrum", J. Math. Anal. Appl., 308:2 (2005), 420-439.

[41] А. Г. Баскаков, "О корректности линейных дифференциальных операторов", $M a$ тем. сб., 190:3 (1999), 3-28; англ. пер.: A. G. Baskakov, "On correct linear differential operators", Sb. Math., 190:3 (1999), 323-348. 
[42] А.Г. Баскаков, А.И. Пастухов, "Спектральный анализ оператора взвешенного сдвига с неограниченными операторными коэффициентами", Сиб. матем. журн., 42:6 (2001), 1231-1243; англ. пер.: A. G. Baskakov, A. I. Pastukhov, "Spectral analysis of a weighted shift operator with unbounded operator coefficients", Siberian Math. J., 42:6 (2001), 1026-1035.

[43] А.Г. Баскаков, "Об обратимости и фредгольмовости разностных операторов", Матем. заметки, 67:6 (2000), 816-827; англ. пер.: A. G. Baskakov, "Invertibility and the Fredholm property of difference operators", Math. Notes, 67:6 (2000), 690-698.

[44] G. Litvinchuk, I. Spitkovskii, Factorization of measurable matrix functions, Oper. Theory Adv. Appl., 25, Birkhäuser Verlag, Basel, 1987, 372 c.

[45] R. Schnaubelt, "Asymptotically autonomous parabolic evolution equations", J. Evol. Equ., 1:1 (2001), 19-37.

[46] А.Г. Баскаков, Гармонический анализ линейных операторов, Изд-во Воронеж. гос. ун-та, Воронеж, 1987, 165 с.

[47] А. Г. Баскаков, “Теория представлений банаховых алгебр, абелевых групп и полугрупп в спектральном анализе линейных операторов", Функциональный анализ, СМФН, 9, МАИ, М., 2004, 3-151; англ. пер.: А. G. Baskakov, "Representation theory for Banach algebras, Abelian groups, and semigroups in the spectral analysis of linear operators", J. Math. Sci. (N. Y.), 137:4 (2006), 4885-5036.

[48] А.Г. Баскаков, "Спектральные критерии почти периодичности решений функциональных уравнений”, Матем. заметки, 24:2 (1978), 195-206; англ. пер.: A. G. Baskakov, "Spectral criteria for almost periodicity of solutions of functional equations", Math. Notes, 24:2 (1978), 606-612.

[49] W. Arendt, C. J. K. Batty, "Tauberian theorems and stability of one-parameter semigroups", Trans. Amer. Math. Soc., 306:2 (1988), 837-852.

[50] Yu. I. Lyubich, Quôc Phóng Vũ, "Asymptotic stability of linear differential equations on Banach spaces", Studia Math., 88:1 (1988), 37-42.

[51] М. С. Бичегкуев, "Об ослабленной задаче Коши для линейного дифференциального включения", Матем. заметки, 79:4 (2006), 483-487; англ. пер.: M. S. Bichegkuev, "On a weakened Cauchy problem for a linear differential inclusion", Math. Notes, 79:3-4 (2006), 449-453.

[52] М. С. Бичегкуев, "Условия разрешимости разностных включений", Изв. РАН. Сер. матем., 72:4 (2008), 25-36; англ. пер.: M.S. Bichegkuev, "Conditions for solubility of difference inclusions", Izv. Math., 72:4 (2008), 647-658.

[53] М. С. Бичегкуев, "Об ограниченных решениях разностных включений", Изв. вузов. Матем., 8 (2008), 16-24; англ. пер.: M. S. Bichegkuev, "On bounded solutions of difference inclusions", Russian Math. (Iz. VUZ), 52:8 (2008), 12-19.

[54] М. С. Бичегкуев, “Линейные разностные и дифференциальные операторы с неограниченными операторными коэффициентами в весовых пространствах", Матем. заметки, 86:5 (2009), 673-680; англ. пер.: M.S. Bichegkuev, "Linear difference and differential operators with unbounded operator coefficients in weight spaces", Math. Notes, 86:5-6 (2009), 637-644.

[55] M.S. Bichegkuev, "On the spectrum of difference and differential operators in weighted spaces", Funct. Anal. Appl., 44:1 (2010), 65-68.

[56] М. С. Бичегкуев, "Об условиях разрешимости разностных уравнений с начальным условием из подпространства”, Сиб. матем. журн., 51:4 (2010), 751-768; англ. пер.: M. S. Bichegkuev, "Solvability conditions for the difference equations with an initial condition in a subspace", Sib. Math. J., 51:4 (2010), 595-609.

[57] М. С. Бичегкуев, С. В. Бесаева, "О спектральных свойствах разностных и дифференциальных операторов в весовых пространствах", Изв. вузов. Матем., 2011, 
№ 2, 16-21; англ. пер.: M. S. Bichegkuev, S. V. Besaeva, "Spectral properties of difference and differential operators in weighted spaces", Russian Math. (Iz. VUZ), 55:2 (2011), 13-17.

[58] М. С. Бичегкуев, "Об условиях обратимости разностных и дифференциальных операторов в весовых пространства”, Изв. РАН. Сер. матем., 75:4 (2011), 3-20; англ. пер.: M.S. Bichegkuev, "On conditions for invertibility of difference and differential operators in weight spaces", Izv. Math., 75:4 (2011), 665-680.

[59] А. Г. Баскаков, Ю.Н. Синтяев, "Разностные операторы в исследовании дифференциальных операторов: оценки решений", Дифферени. уравнения, 46:2 (2010), 210-219; англ. пер.: A. G. Baskakov, Yu. N. Sintyaev, "Finite-difference operators in the study of differential operators: Solution estimates", Differ. Equ., 46:2 (2010), 214-223.

[60] L. Gearhart, "Spectral theory for contraction semigroups on Hilbert spaces", Trans. Amer. Math. Soc., 236 (1978), 385-394.

[61] J. Prüss, "On the spectrum of $C_{0}$-semigroups", Trans. Amer. Math. Soc., 284:2 (1984), 847-857.

[62] А.Г. Баскаков, "Некоторые условия обратимости линейных дифференциальных и разностных операторов", Докл. РАН, $333: 3$ (1993), 282-284; англ. пер.: A. G. Baskakov, "Some conditions for the invertibility of linear differential and difference operators", Russian Acad. Sci. Dokl. Math., 48:3 (1994), 498-501.

[63] А.Г. Баскаков, “Оценки ограниченных решений линейных дифференциальных уравнений”, Дифферени. уравнения, 39:3 (2003), 413-415; англ. пер.: A. G. Baskakov, "Estimates for bounded solutions of linear differential equations", Differ. Equ., 39:3 (2003), 447-450.

[64] А. Г. Баскаков, К. С. Кобычев, “Оценки оператора вложения пространства Соболева периодических функций и оценки решений дифференциальных уравнений с периодическими коэффициентами", Дифферени. уравнения, 47:5 (2011), 611-620; англ. пер.: А. G. Baskakov, K. S. Kobychev, "Estimates for the embedding operator of a Sobolev space of periodic functions and for the solutions of differential equations with periodic coefficients", Differ. Equ., 47:5 (2011), 609-619.

[65] R. Schnaubelt, "Sufficient conditions for exponential stability and dichotomy of evolution equations", Forum Math., 11:5 (1999), 543-566.

[66] R. Schnaubelt, "Well-posedness and asymptotic behaviour of non-autonomous linear evolution equations", Evolution equations, semigroups and functional analysis (Milano, 2000), Progr. Nonlinear Differential Equations Appl., 50, Birkhäuser, Basel, 2002, 311-338.

[67] А. Г. Баскаков, М. К. Чернышов, "Некоторые условия обратимости дифференциальных операторов второго порядка", Укр. матем. журн., 47:3 (1995), 411-413; англ. пер.: А. G. Baskakov, M. K. Chernyshov, "On the invertibility of differential operators of the second order", Ukrainian Math. J., 47:3 (1995), 477-480.

[68] А. Ю. Левин, “Теорема Харитонова для слабо нестационарных систем”, УМН, 50:6(306) (1995), 189-190; англ. пер.: A. Yu. Levin, "Kharitonov's theorem for weakly non-stationary systems", Russian Math. Surveys, 50:6 (1995), 1280-1281.

[69] А. Г. Баскаков, В.В.Юргелас, "Индефинитная диссипативность и обратимость линейных дифференциальных операторов", Укр. матем. журн., 41:12 (1989), 1613-1618; англ. пер.: A. G. Baskakov, V.V. Yurgelas, "Indefinite dissipativity and invertibility of linear differential operators", Ukrainian Math. J., 41:12 (1989), 1385-1389.

[70] А. Г. Баскаков, “Дихотомия спектра несамосопряженных операторов", Сиб. матем. журн., 32:3 (1991), 24-30; англ. пер.: A. G. Baskakov, "Dichotomy of the spectrum of non-self-adjoint operators", Siberian Math. J., 32:3 (1991), 370-375. 
[71] А. Г. Баскаков, В. В. Юргелас, "Круговая дихотомия спектра одного класса несамосопряженных операторов", Изв. вузов. Матем., 1994, № 10, 12-18; англ. пер.: A. G. Baskakov, V.V. Yurgelas, "Circular dichotomy of the spectrum of a certain class of non-self-adjoint operators", Russian Math. (Iz. VUZ), 38:10 (1994), 10-15.

[72] В. Н. Фомин, Математическая теория параметрического резонанса в линейных распределенных системах, Изд-во ЛГУ, Л., 1972, 240 с.

[73] А.Г. Баскаков, Замена Крылова-Боголюбова в теории нелинейных возмущений линейнъх операторов, Препринт № 80-19, Ин-т математики АН УССР, Киев, 1980, $44 \mathrm{c}$.

[74] А.Г. Баскаков, "Спектральный анализ возмущенных неквазианалитических и спектральных операторов", Изв. РАН. Сер. матем., 58:4 (1994), 3-32; англ. пер.: A. G. Baskakov, "Spectral analysis of perturbed nonquasianalytic and spectral operators", Russian Acad. Sci. Izv. Math., 45:1 (1995), 1-31.

[75] А. Г. Баскаков, "Теорема о расщеплении оператора и некоторые смежные вопросы аналитической теории возмущений”, Изв. АН СССР. Сер. матем., 50:3 (1986), 435-457; англ. пер.: A. G. Baskakov, "A theorem on splitting an operator, and some related questions in the analytic theory of perturbations", Math. USSR-Izv., 28:3 (1987), 421-444.

[76] A. G. Baskakov, "On the decomposition of a perturbed operator of weighted shift", Ukrainian Math. J., 50:2 (1998), 177-185.

[77] А. Г. Баскаков, А. В. Дербушев, А. О. Щербаков, "Метод подобных операторов в спектральном анализе несамосопряженного оператора Дирака с негладким потенциалом", Изв. РАН. Сер. матем., 75:3 (2011), 3-28; англ. пер.: A. G. Baskakov, A. V. Derbushev, A. O. Shcherbakov, "The method of similar operators in the spectral analysis of non-self-adjoint Dirac operators with non-smooth potentials", Izv. Math., 75:3 (2011), 445-469.

[78] А.Г. Баскаков, "Расщепление возмущенного дифференциального оператора с неограниченными операторными коэффициентами", Фундамент. и прикл. матем., 8:1 (2002), 1-16.

[79] С. Г. Крейн, "Асимптотическое расщепление операторных уравнений”, Функиии Ляпунова и их применения, Наука, Новосибирск, 1986, 206-214.

[80] Н. Д. Копачевский, С. Г. Крейн, Нго Зуй Кан, Операторные методы в линейной гидродинамике. Эволюиионные и спектральные задачи, Наука, М., 1989, 416 с.

[81] F. Holland, "Harmonic analysis on amalgams of $L^{p}$ and $\ell^{q} "$, J. London Math. Soc. (2), 10:3 (1975), 295-305.

[82] N. Wiener, "On the representation of functions by trigonometrical integrals", Math. Z., 24:1 (1926), 575-616.

[83] N. Wiener, "Tauberian theorems", Ann. of Math. (2), 33:1 (1932), 1-100.

[84] W. Stepanoff, "Über einige Verallgemeinerung der fast periodischen Functionen", Math. Ann., 95:1 (1926), 473-498.

[85] J. J. F. Fournier, J. Stewart, "Amalgams of $L^{p}$ and $\ell^{q}$ ", Bull. Amer. Math. Soc. (N.S.), 13:1 (1985), 1-21.

[86] А.Г. Баскаков, А.А. Воробьев, М. Ю. Романова, "Гиперболические полугруппы операторов и уравнение Ляпунова", Матем. заметки, 89:2 (2011), 190-203; англ. пер.: A. G. Baskakov, A. A. Vorob'ev, M. Yu. Romanova, "Hyperbolic operator semigroups and Lyapunov's equation", Math. Notes, 89:1-2 (2011), 194-205.

[87] Nguen Thieu Huy, "Exponential dichotomy of evolution equations and admissibility of function spaces on a half-line", J. Funct. Anal., 235:1 (2006), 330-354.

[88] Р. Эдвардс, Функиионалъный анализ. Теория и приложения, Мир, М., 1969, 1071 c.; пер. с англ.: R. E. Edwards, Functional analysis. Theory and applications, Holt, Rinehart and Winston, New York-Toronto-London, 1965, xiii+781 pp. 
[89] В. М. Тюрин, "Об обратимости линейных дифференциальных операторов в некоторых функциональных пространствах", Сиб. матем. журн., 32:3 (1991), 160-165; англ. пер.: V. M. Tyurin, "Invertibility of linear differential operators in certain functional spaces", Siberian Math. J., 32:3 (1991), 485-490.

[90] A. G. Baskakov, "Investigation of spectral properties of the differential operators $\mathscr{L}=$ $-d / d t+A(t)$ by means of the semigroup $\exp \mathscr{L} t, t \geqslant 0 "$, International conference on functional differential equations and applications (Moscow, Russia, August 14-21, 1994), 8-9.

[91] Yu. Latushkin, Yu. Tomilov, "Fredholm properties of evolution semigroups", Illinois J. Math., 48:3 (2004), 999-1020.

[92] M. Megan, A. L. Sasu, B. Sasu, "Discrete admissible and exponential dichotomy for evolution families", Discrete Contin. Dyn. Syst., 9:2 (2003), 383-397.

[93] I. Gochberg, S. Goldberg, M. A. Kaashoek, Classes of linear operators, v. I, Oper. Theory Adv. Appl., 49, Birkhäuser Verlag, Basel, 1990, xiv+468 pp.

[94] S. Goldberg, Unbounded linear operators. Theory and applications, McGraw-Hill Book Co., New York-Toronto-London, 1966, viii+199 pp.

[95] A. Ben-Artzi, I. Gochberg, M. A. Kaashoek, "Invertibility and dichotomy of differential operators on a half-line", J. Dynam. Differential Equations, 5:1 (1993), 1-36.

[96] В.Б. Диденко, "О спектральных свойствах дифференциальных операторов с неограниченными операторными коэффициентами, определяемых линейным отношением", Матем. заметки, 89:2 (2011), 226-240; англ. пер.: V. B. Didenko, "On the spectral properties of differential operators with unbounded operator coefficients determined by a linear relation", Math. Notes, 89:1-2 (2011), 224-237.

[97] В.А.Плисс, "Ограниченные решения однородных линейных систем дифференциальных уравнений”, Проблемы асимптотической теории нелинейных колебаний, Наукова думка, Киев, 1977, 168-173.

А. Г. Баскаков (А. G. Baskakov)

Воронежский государственный университет

E-mail: anatbaskakov@yandex.ru
Поступила в редакцию

31.10 .2012 